\title{
Cation-Modulated Electron Transfer Channel: H-atom Transfer vs Proton-Coupled Electron Transfer with Variable Electron Transfer Channel in Acylamide Units
}

\author{
Xiaohua Chen, Yuxiang Bu* \\ Contribution from Institute of Theoretical Chemistry, Shandong University, Jinan, 250100, P. \\ R. China
}

\section{Supporting Information}

\section{Complete citation for Reference 29:}

Frisch, M. J.; Trucks, G. W.; Schlegel, H. B.; Scuseria, G. E.; Robb, M. A.; Cheeseman, J. R.; Montgomery, Jr., J. A.; Vreven, T.; Kudin, K. N.; Burant, J. C.; Millam, J. M.; Iyengar, S. S.; Tomasi, J.; Barone, V.; Mennucci, B.; Cossi, M.; Scalmani, G.; Rega, N.; Petersson, G. A.; Nakatsuji, H.; Hada, M.; Ehara, M.; Toyota, K.; Fukuda, R.; Hasegawa, J.; Ishida, M.; Nakajima, T.; Honda, Y.; Kitao, O.; Nakai, H.; Klene, M.; Li, X.; Knox, J. E.; Hratchian, H. P.; Cross, J. B.; Bakken, V.; Adamo, C.; Jaramillo, J.; Gomperts, R.; Stratmann, R. E.; Yazyev, O.; Austin, A. J.; Cammi, R.; Pomelli, C.; Ochterski, J. W.; Ayala, P. Y.; Morokuma, K.; Voth, G. A.; Salvador, P.; Dannenberg, J. J.; Zakrzewski, V. G.; Dapprich, S.; Daniels, A. D.; Strain, M. C.; Farkas, O.; Malick, D. K.; Rabuck, A. D.; Raghavachari, K.; Foresman, J. B.; Ortiz, J. V.; Cui, Q.; Baboul, A. G.; Clifford, S.; Cioslowski, J.; Stefanov, B. B.; Liu, G.; Liashenko, A.; Piskorz, P.; Komaromi, I.; Martin, R. L.; Fox, D. J.; Keith, T.; Al-Laham, M. A.; Peng, C. Y.; Nanayakkara, A.; Challacombe, M.; Gill, P. M. W.; Johnson, B.; Chen, W.; Wong, M. W.; Gonzalez, C.; and Pople, J. A. Gaussian 03, Revision C.02; Gaussian, Inc.: Wallingford, CT, 2004.

\footnotetext{
*The corresponding author: Yuxiang Bu, e-mail: byx@sdu.edu.cn
} 


\section{Additional Simple Structural Remarks}

To determine the effect of the alkali metal cations on the FF PT/ET self-exchange reaction, the associations of the hydrated and unhydrated alkali metal cations $\left(\mathrm{Na}^{+}, \mathrm{K}^{+}\right)$with FF at its two oxygen atoms have been optimized. Figure S2 shows the structures of the three sodium ion complexes and their corresponding transition states. Besides, only the most important geometrical parameters of them are presented in Figure S2. Structural analyses reveal several interesting variations. Firstly, for reactants, the lengths of $\mathrm{N} \cdots \mathrm{HN}$ shorten from FF-Na ${ }^{+}(3.56 \AA)$, FF-Na ${ }^{+}\left(\mathrm{H}_{2} \mathrm{O}\right)_{1}(3.38 \AA)$ to $\mathbf{F F}-\mathrm{Na}^{+}\left(\mathrm{H}_{2} \mathrm{O}\right)_{2}(3.31 \AA)$. However, the lengths of two $\mathrm{Na}^{+} \cdots \mathrm{O}$ become longer from FF-Na ${ }^{+}(2.15 \AA, 2.25 \AA), \mathbf{F F}-\mathrm{Na}^{+}\left(\mathrm{H}_{2} \mathrm{O}\right)_{1}$ $(2.17 \AA, 2.30 \AA)$ to $\mathbf{F F}-\mathrm{Na}^{+}\left(\mathrm{H}_{2} \mathrm{O}\right)_{2}(2.21 \AA, 2.35 \AA)$. This can be understood in terms of attractive Coulomb interactions that stabilize the reactants over the intramolecular charge migration. Secondly, for their corresponding transition states, one of salient structural characters is that all the atoms in the active site (FF) are coplanar or nearly coplanar. Besides, it should be noted that the $\mathrm{N}^{\cdots} \mathrm{H} \cdot \mathrm{N}$ and $\mathrm{N} \cdots \mathrm{H}$ distances remain constant, 2.55 and $1.28 \AA$, respectively, and the $\mathrm{O} \cdots \mathrm{O}$ distances are nearly equal. Therefore, in Figure $\mathrm{S} 2$, the proton of the amino nitrogen can easily be migrated due to the short $\mathrm{N}^{\cdots} \mathrm{H} \cdots \mathrm{N}$ length of $2.55 \AA$ in their transition states. For the potassium ion complexes, a similar phenomenon to the sodium ion complexes can be found.

Figure S3 displays the geometrical structures for the five hydrated calcium-coupled FF modes and their corresponding transition states. For the reactant complexes, the remarkable changes are the shortening of the $\mathrm{N} \cdots \mathrm{HN}$ and $\mathrm{O} \cdots \mathrm{O}$ distances with the increase of water ligands. Their corresponding transition states show very similar structure features, cyclic seven-membered structure $\mathrm{O}=\mathrm{C}-\mathrm{N} \cdots \mathrm{H}^{\cdots} \mathrm{N}-\mathrm{C}=\mathrm{O}$, with the nearly equal $\mathrm{N} \cdots \mathrm{H} \cdots \mathrm{N}$ distances in

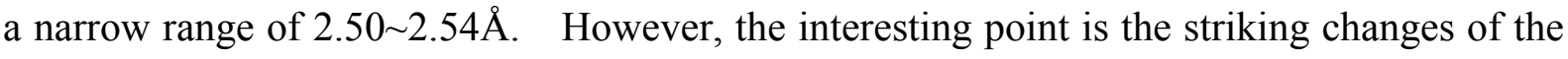
$\mathrm{O} \cdots \mathrm{O}$ distances, shortening with increasing water ligands (see Figure S3, 2.99 $\left(\mathbf{F F}^{\text {ts }}-\mathrm{Ca}^{2+}\left(\mathrm{H}_{2} \mathrm{O}\right)_{1}\right) \approx 3.00 \AA \quad\left(\mathbf{F F}^{\text {ts }}-\mathrm{Ca}^{2+}\left(\mathrm{H}_{2} \mathrm{O}\right)_{2}\right)>2.81 \AA\left(\mathbf{F F}^{\text {ts }}-\mathrm{Ca}^{2+}\left(\mathrm{H}_{2} \mathrm{O}\right)_{3}\right)>2.66 \AA$ $\left.\left(\mathbf{F F}^{\text {ts }}-\mathrm{Ca}^{2+}\left(\mathrm{H}_{2} \mathrm{O}\right)_{4}\right)>2.48 \AA\left(\mathbf{F F}^{\text {ts }}-\mathrm{Ca}^{2+}\left(\mathrm{H}_{2} \mathrm{O}\right)_{5}\right)\right)$. For $\mathbf{F F}^{\text {ts }}-\mathrm{Ca}^{2+}\left(\mathrm{H}_{2} \mathrm{O}\right)_{1}$ and $\mathbf{F F}^{\text {ts }}-\mathrm{Ca}^{2+}\left(\mathrm{H}_{2} \mathrm{O}\right)_{2}$, all atoms in the FF moieties are not coplanar. The dihedral angles, $D_{\mathrm{H} 6-\mathrm{N} 3-\mathrm{H} 5-\mathrm{C} 1}$ and $D_{\mathrm{H} 11-\mathrm{N} 10-\mathrm{H} 5-\mathrm{C} 8}$, are both $167.5^{\circ}$ for $\mathbf{F F}^{\mathrm{ts}}-\mathrm{Ca}^{2+}\left(\mathrm{H}_{2} \mathrm{O}\right)_{1}$ and $167.1^{\circ}$ for $\mathbf{F F}^{\mathrm{Ts}}-\mathrm{Ca}^{2+}\left(\mathrm{H}_{2} \mathrm{O}\right)_{2}$. But for the other three transition states, all the atoms in the FF moieties are coplanar. The same 
trend can be found for the hydrated magnesium-coupled FF systems.

\section{Remarks for choice of calculational methods}

In addition, to further verify the applicability of the B3LYP/6-311++G** method, some other calculational methods were also used which are B3LYP, MPW1PW91, PBE1PBE, B3PW91, BHandHLYP and MP2 with aug-cc-PVDZ basis set. The relevant structural parameters for $\mathbf{F}$ and $\mathbf{F r}$ are given in Table S3, together with other experimental and theoretical values. The deviations of all theoretical values from their corresponding experimental values are $-0.02 \sim+0.01 \AA$ for $\mathrm{C}-\mathrm{O}$ bond and $0.01 \AA$ for $\mathrm{C}-\mathrm{N}$ bond, respectively. In particular, for B3LYP method, the yielded errors are $0.01 \AA(\mathrm{C}-\mathrm{O})$ and $0.01 \AA(\mathrm{C}-\mathrm{N})$. The very small deviations and excellent agreement among them have indicated that it is appropriate to use the UB3LYP method with $6-311++\mathrm{G}^{* *}$ basis set to probe the ET/PT mechanism of the FF pair and FF coupled with a series of hydrated metal cations.

No experimental data have been reported for FF complex and its relevant radical exchange mechanism. So, we also determined the FF complex and its $\mathbf{F F}^{\text {ts }}$ transition state structure at different levels of theory. The relevant geometrical parameters, spin densities in the transition state and the binding energies in the reactant are listed in Table S4. The deviations among several methods are within $0.05 \AA(\mathrm{O} \ldots \mathrm{O}), 0.01 \AA(\mathrm{N} \ldots \mathrm{H} \ldots \mathrm{N}), 0.01 \AA$ $(\mathrm{N} \ldots \mathrm{H}), 0.5^{\circ}\left(\mathrm{A}_{\mathrm{NHN}}\right)$ for geometrical parameters, and $1.3 \mathrm{kcal} / \mathrm{mol}\left(\Delta \mathrm{E}_{\mathrm{a}}\right), 1.5 \mathrm{kcal} / \mathrm{mol}\left(\Delta \mathrm{E}_{\mathrm{b}}\right)$, respectively. The deviations of $\rho_{\mathrm{N}}$ and $\rho_{\mathrm{O}}$ are almost equal to zero. Together with the calculated geometrical parameters for $\mathbf{F}$ and $\mathbf{F r}$, all these data indicate B3LYP/6-311++G** method can yield values that are in good agreement with those from other methods. Therefore, the UB3LYP/6-311++G** was used to predict the PT/ET mechanism of FF reaction under different conditions.

\section{Remarks for Monomers and Their Complexes}

It should be noted that Fr has two stable conformers (see below figure). The isomer Fr1 bearing cis- $(\mathrm{N}) \mathrm{H}$ with respect to the $\mathrm{C}=\mathrm{O}$ oxygen is the most stable, while another isomer (Fr2) bearing the near-trans-(N)H is energetically higher by $\sim 3.0 \mathrm{kcal} / \mathrm{mol}$ than the Fr1 isomer. 
Selected geometrical parameters for formamide (F) and two isomers of formamidic radical (Fr1 and Fr2) are referred to the values calculated here at the B3LYP/6-311++G** level, experimental values (in brackets, from Sugisaki, R.; Tanaka, T.; Hirota, E. J .Mol. Spectrosc. 1974, 49, 294.), other theoretical values (in parentheses, from Cabaleiro-Lago, E. M.; Otero, J. R. J. Chem. Phys. 2002, 117, 1621.). The bond distances are in $\AA$ and intramolecular angles are in deg. The B3LYP/6-311++G** method can yield the results very consistent with the experimental values.

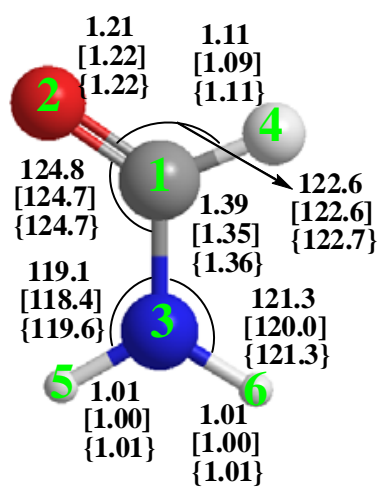

$\mathbf{F}$

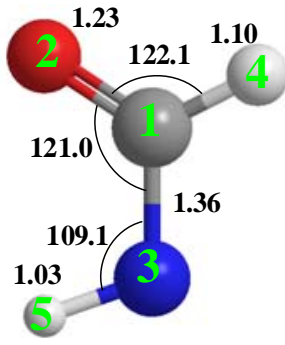

Fr1 (cis- H)

Dihedral angles

Relative energies $/ \mathrm{kcal} / \mathrm{mol}$

Spin densities

Radical-type $\angle 2135=25.9^{\circ}$

0.0

$\rho_{\mathrm{N}}=0.82 / \rho_{\mathrm{O}}=0.35$

$\pi$-type

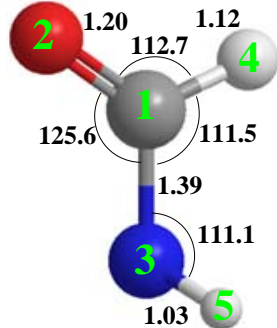

Fr2 (trans- $H)$

$\angle 2135=113.6^{\circ}$

3.0

$\rho_{\mathrm{N}}=1.00 / \rho_{\mathrm{O}}=0.02$

$\sigma$-type

Only the later (Fr2) can form the cyclic FF mode with formamide (F) for favoring a particular PCET mechanism with proton transfer via the N...H...N vector and electron

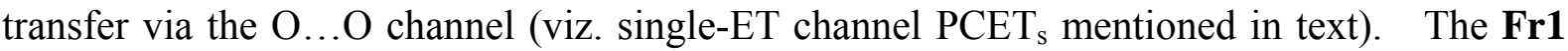
(cis- $(\mathrm{N}) \mathrm{H}$ ) can only form a noncyclic structure with $\mathbf{F}$ (see the following noncyclic FF structure) in which PT/ET exchanges occur as Hydrogen Atom Transfer (HAT) via the N...H...N vector. The non-cyclic FF is $0.7 \mathrm{kcal} / \mathrm{mol}$ slightly more stable than the cyclic FF for reactant states, but for their corresponding transition states, the cyclic $\mathbf{F} \mathbf{F}^{\mathbf{t s}}$ is significantly lower by $5.3 \mathrm{kcal} / \mathrm{mol}$ than the non-cyclic $\mathbf{F F}^{\mathbf{t s}}$. As a result, the activation barrier height (4.3 $\mathrm{kcal} / \mathrm{mol})$ of the cyclic FF mode is significantly lower by $\sim 6.0 \mathrm{kcal} / \mathrm{mol}$ than that $(10.3$ kcal/mol) of the non-cyclic FF mode. 


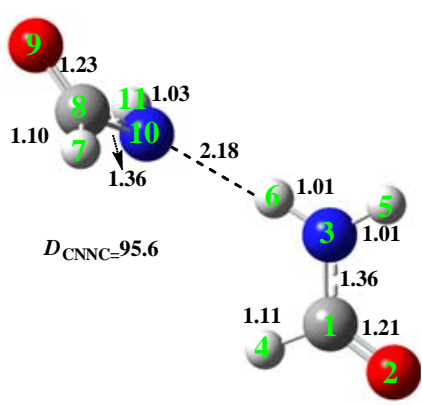

Non-cyclic FF

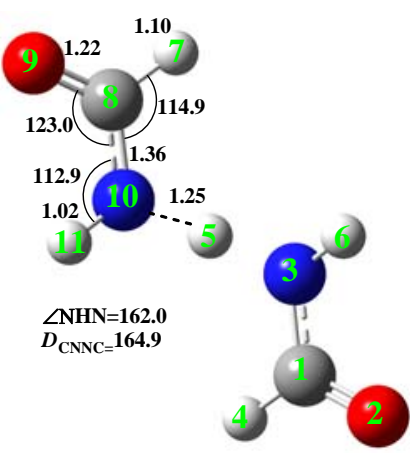

non-cyclic $\mathbf{F F}^{\text {ts }}$

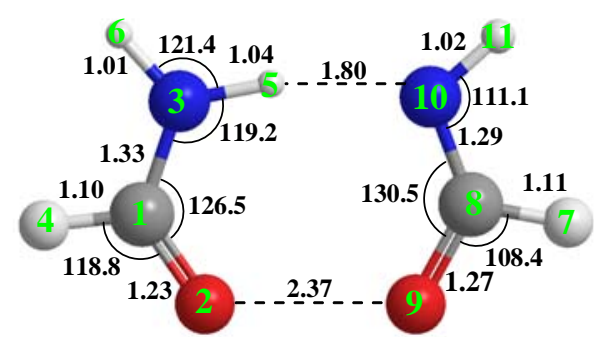

cyclic FF

For the non-cyclic FF mode, the proton/electron exchange always via HAT mechanism, not exhibiting the variable PT/ET cooperative pathway. That is, metal ion coupling only changes the HAT activation barrier height, and can not change its HAT mechanism. However, the cyclic FF mode may exhibit significant change of PT/ET mechanism from HAT to double-ET channel PCET $\mathrm{d}_{\mathrm{d}}$ to single-ET channel $\mathrm{PCET}_{\mathrm{s}}$ or vise versa. This is an interesting character. Therefore, the focus of this work is on the cation modulation of PT/ET mechanism associating with this special binding mode, cyclic FF. We only discuss the structures associating with cyclic FF mode formed by $\mathbf{F}$ and $\mathbf{F r} 2$ (we call $\mathbf{F r} 2$ as $\mathbf{F r}$ in the text).

\section{Main figures considered in this work}

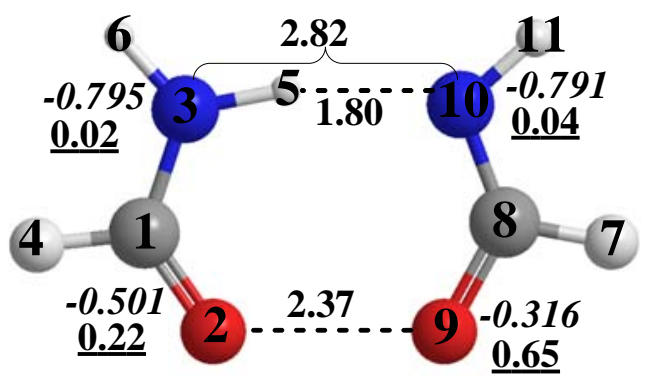

FF

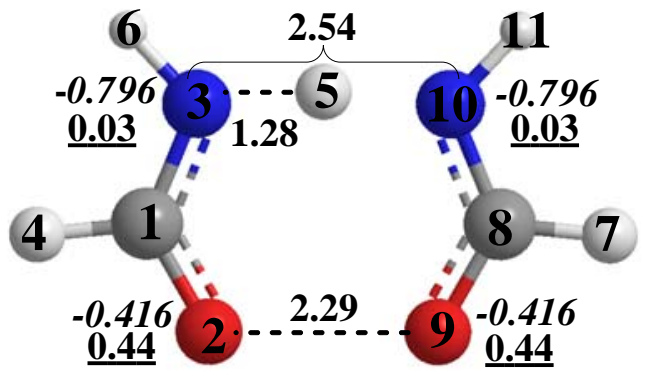

$\mathbf{F F}^{\text {ts }}$

Figure S1. The optimized structures of the cisoid coupling mode for the F...Fr complexes and its PT/ET transition state with main parameters. The bond distances are in $\AA$; the italic numbers are the natural atomic charges (in $e$ ) obtained from the Natural population analyses and the underlined numbers are atomic spin densities. Remarks for monomers and other 
coupling mode are given above (Remark 4).
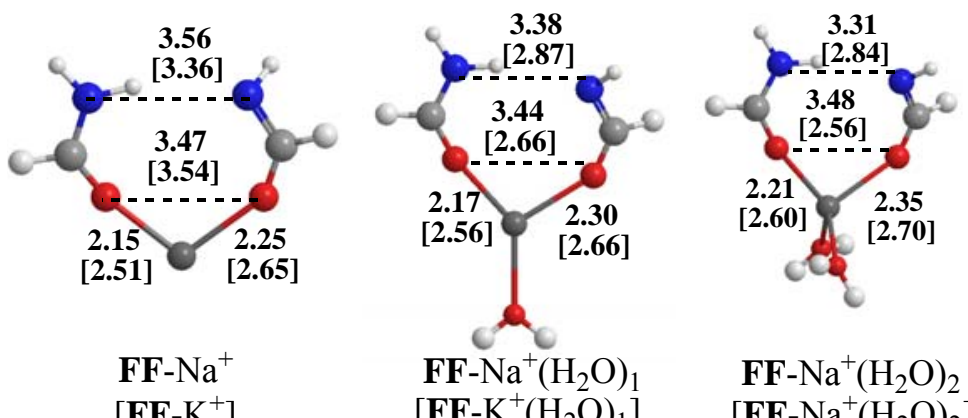

FF- $\mathrm{Na}^{+}\left(\mathrm{H}_{2} \mathrm{O}\right)_{2}$

$\left[\mathrm{FF}-\mathrm{K}^{+}\right]$

$\left[\mathbf{F F}-\mathrm{K}^{+}\left(\mathrm{H}_{2} \mathrm{O}\right)_{1}\right]$
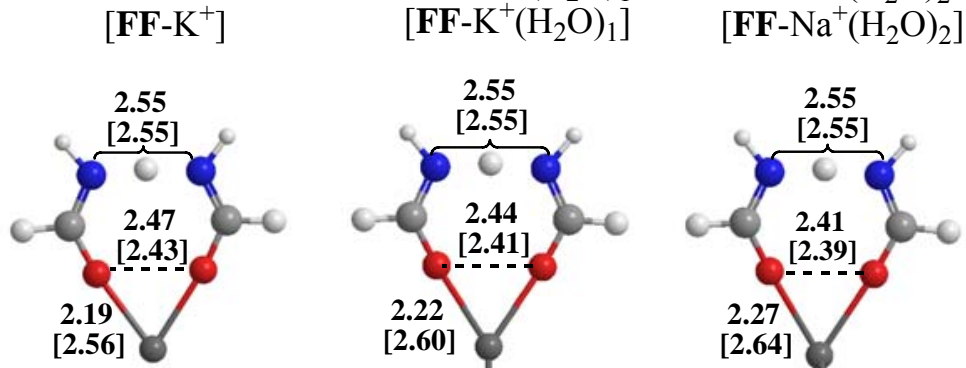

$$
\text { FF }^{\text {ts }}-\mathrm{Na}^{+}
$$

$\left[\mathbf{F F}^{\mathrm{ts}}-\mathrm{K}^{+}\right]$
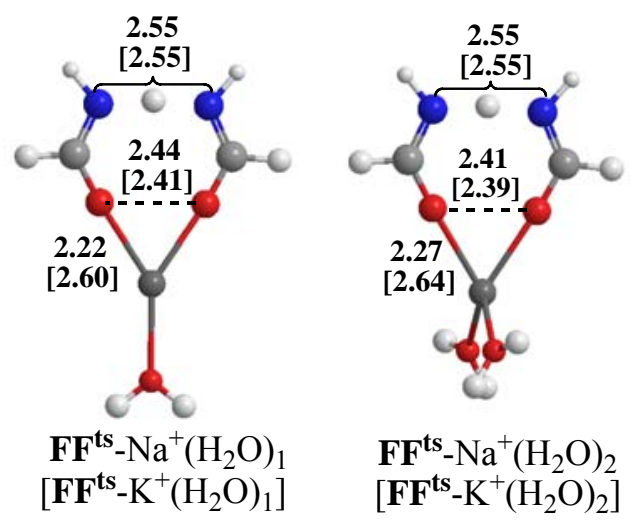

$\mathbf{F F}^{\text {ts }}-\mathrm{Na}^{+}\left(\mathrm{H}_{2} \mathrm{O}\right)_{2}$

$\left[\mathbf{F F}^{\mathbf{t s}}-\mathrm{K}^{+}\left(\mathrm{H}_{2} \mathrm{O}\right)_{2}\right]$

Figure S2. The three sodium ion (potassium in square brackets) complexes and corresponding transition state structures obtained from the UB3LYP/6-311++G** calculations. The bond distances are in $\AA$. 

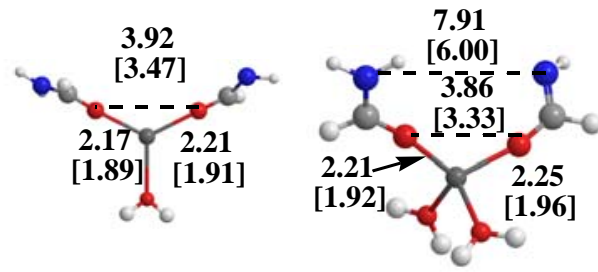

FF- $\mathrm{Ca}^{2+}\left(\mathrm{H}_{2} \mathrm{O}\right)_{1}$ $\left[\right.$ FF- $-\mathrm{Mg}^{2+}\left(\mathrm{H}_{2} \mathrm{O}\right)_{1}$ ]

$\left[\right.$ FF- $\mathrm{Mg}^{2+}\left(\mathrm{H}_{2} \mathrm{O}\right)_{2}$ ]

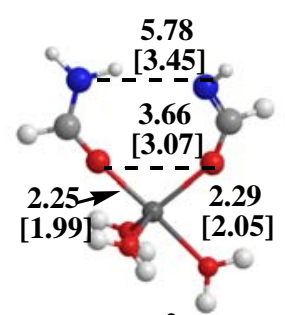

FF- $-\mathrm{Ca}^{2+}\left(\mathrm{H}_{2} \mathrm{O}\right)_{3}$

$\left[\right.$ FF- $\left.-\mathrm{Mg}^{2+}\left(\mathrm{H}_{2} \mathrm{O}\right)_{3}\right]$

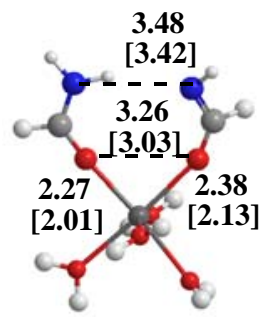

FF- $-\mathrm{Ca}^{2+}\left(\mathrm{H}_{2} \mathrm{O}\right)_{4}$

$\left[\right.$ FF- $\left.\mathrm{Mg}^{2+}\left(\mathrm{H}_{2} \mathrm{O}\right)_{4}\right]$

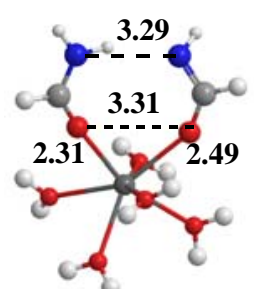

FF- $-\mathrm{Ca}^{2+}\left(\mathrm{H}_{2} \mathrm{O}\right)_{5}$
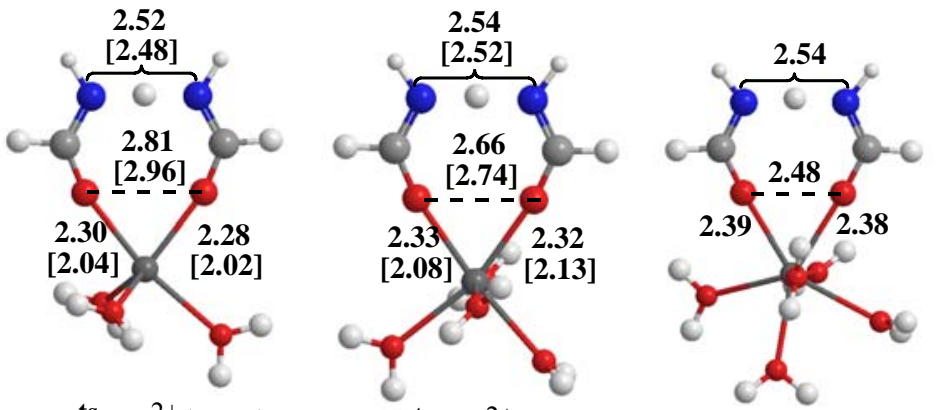

$\mathbf{F F}^{\text {ts }}-\mathrm{Ca}^{2+}\left(\mathrm{H}_{2} \mathrm{O}\right)_{1} \quad \mathbf{F F}^{\text {ts }}-\mathrm{Ca}^{2+}\left(\mathrm{H}_{2} \mathrm{O}\right)_{2}$

$\left[\mathbf{F F}^{\text {ts }}-\mathrm{Mg}^{2+}\left(\mathrm{H}_{2} \mathrm{O}\right)_{1}\right] \quad\left[\mathbf{F F}^{\text {ts }}-\mathrm{Mg}^{2+}\left(\mathrm{H}_{2} \mathrm{O}\right)_{2}\right]$

$\mathbf{F F}^{\text {ts }}-\mathrm{Ca}^{2+}\left(\mathrm{H}_{2} \mathrm{O}\right)_{3}$

$\left[\mathbf{F F}^{\text {ts }}-\mathrm{Mg}^{2+}\left(\mathrm{H}_{2} \mathrm{O}\right)_{3}\right]$

$\mathbf{F F}^{\text {ts }}-\mathrm{Ca}^{2+}\left(\mathrm{H}_{2} \mathrm{O}\right)_{4}$

$\left[\mathbf{F F}^{\text {ts }}-\mathrm{Mg}^{2+}\left(\mathrm{H}_{2} \mathrm{O}\right)_{4}\right]$

Figure S3. The five calcium (four magnesium in square brackets) complexes and corresponding transition state structures obtained from the UB3LYP/6-311++G** calculations. Interestingly, the $\mathrm{N}-\mathrm{H}^{\cdots} \mathrm{N}$ distances shorten with the increase of water ligands for the same cation. However, the $\mathrm{N}^{\cdots} \mathrm{H} \cdots \mathrm{N}$ and $\mathrm{N} \cdots \mathrm{H}$ distances are nearly equal in the five transition state structures. In addition, it is worth noting that the $\mathrm{O} \cdots \mathrm{O}$ distances shorten from $\mathbf{F F}^{\text {ts }}-\mathrm{Ca}^{2+}\left(\mathrm{H}_{2} \mathrm{O}\right)$ to $\mathbf{F F}^{\text {ts }}-\mathrm{Ca}^{2+}\left(\mathrm{H}_{2} \mathrm{O}\right)_{5}$ (or from $\mathbf{F F}^{\text {ts }}-\mathrm{Mg}^{2+}\left(\mathrm{H}_{2} \mathrm{O}\right)$ to $\left.\mathbf{F} \mathbf{F}^{\text {ts }}-\mathrm{Mg}^{2+}\left(\mathrm{H}_{2} \mathrm{O}\right)_{4}\right)$. 


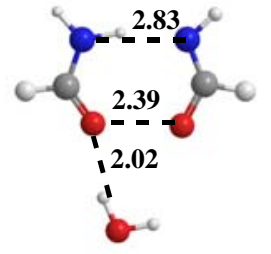

FF- $\left(\mathrm{H}_{2} \mathrm{O}\right)_{1}$

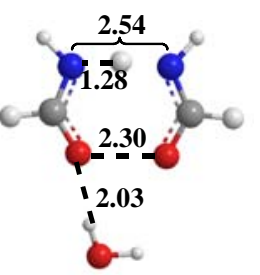

$\mathbf{F F}^{\text {ts }}-\left(\mathrm{H}_{2} \mathrm{O}\right)_{1}$

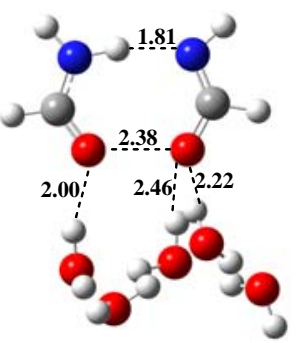

FF- $\left(\mathrm{H}_{2} \mathrm{O}\right)_{5}$

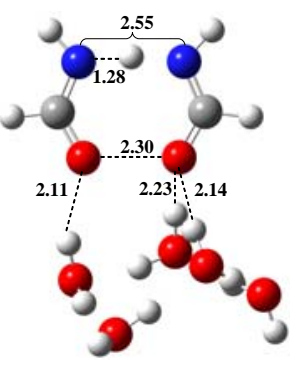

FF $^{\text {ts }}-\left(\mathrm{H}_{2} \mathrm{O}\right)_{5}$

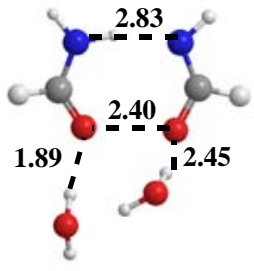

FF- $\left(\mathrm{H}_{2} \mathrm{O}\right)_{2}$

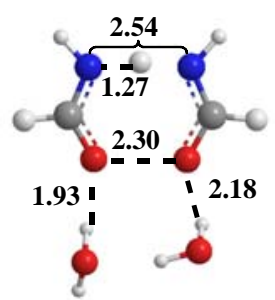

FF $^{\text {ts }}-\left(\mathrm{H}_{2} \mathrm{O}\right)_{2}$

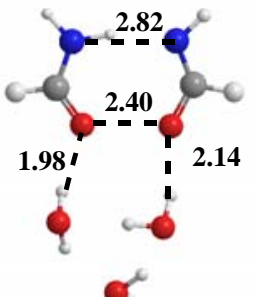

FF- $\left(\mathrm{H}_{2} \mathrm{O}\right)_{3}$
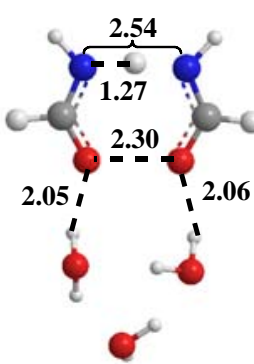

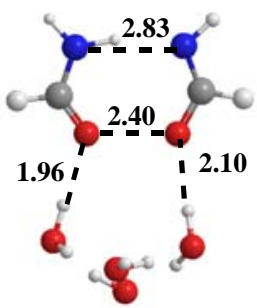

FF- $\left(\mathrm{H}_{2} \mathrm{O}\right)_{4}$

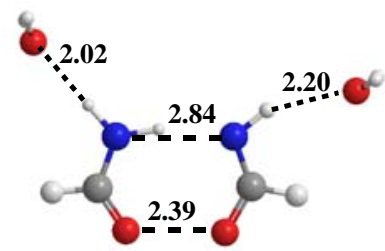

FF-N- $\left(\mathrm{H}_{2} \mathrm{O}\right)_{2}$

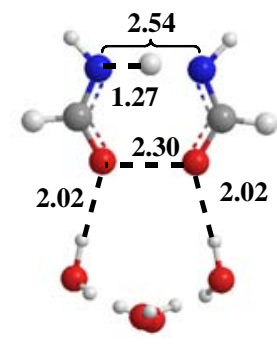

FF $^{\text {ts }}-\left(\mathrm{H}_{2} \mathrm{O}\right)_{4}$

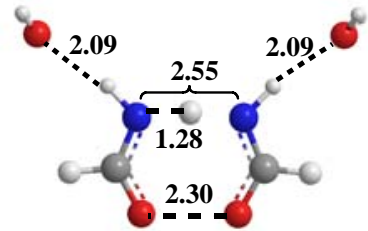

$\mathbf{F F}^{\text {ts }}-\mathrm{N}-\left(\mathrm{H}_{2} \mathrm{O}\right)_{2}$

$\mathbf{F F}^{\text {ts }}-\left(\mathrm{H}_{2} \mathrm{O}\right)_{3}$

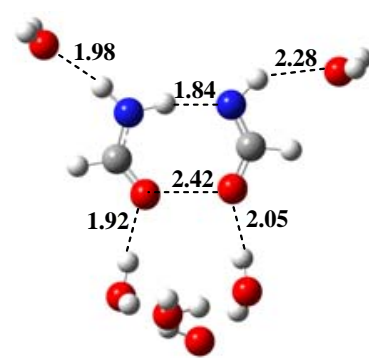

FF- $\left(\mathrm{H}_{2} \mathrm{O}\right)_{4}-\mathrm{N}-\left(\mathrm{H}_{2} \mathrm{O}\right)_{2}$

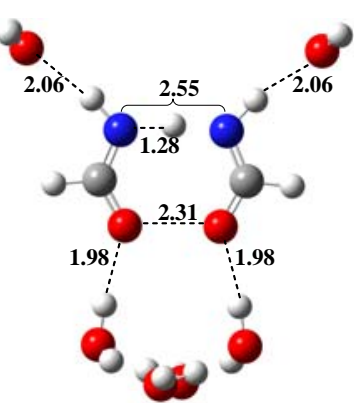

FF $^{\text {ts }}-\left(\mathrm{H}_{2} \mathrm{O}\right)_{4}-\mathrm{N}-\left(\mathrm{H}_{2} \mathrm{O}\right)_{2}$

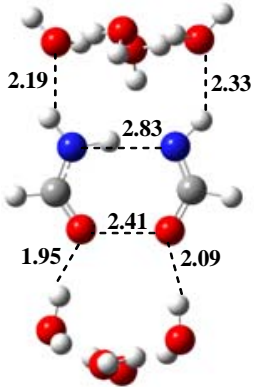

FF- $\left(\mathrm{H}_{2} \mathrm{O}\right)_{4}-\mathrm{N}-\left(\mathrm{H}_{2} \mathrm{O}\right)_{4}$

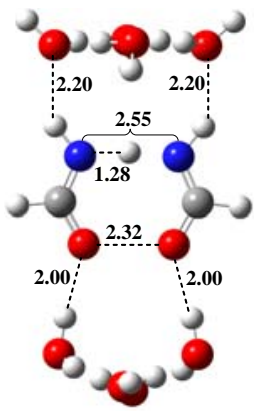

$\mathbf{F F}^{\text {ts }}-\left(\mathrm{H}_{2} \mathrm{O}\right)_{4}-\mathrm{N}-\left(\mathrm{H}_{2} \mathrm{O}\right)_{4}$

Figure S4. The different water-coupled FF complexes and corresponding transition state structures obtained from the UB3LYP/6-311++G** calculations. It is obvious that the active site (FF) keeps a similar structure not only in the transition state structures but also in the reactants with the increase of water molecules. Therefore, water solvation can not influence the FF PT/ET reaction mechanism. 


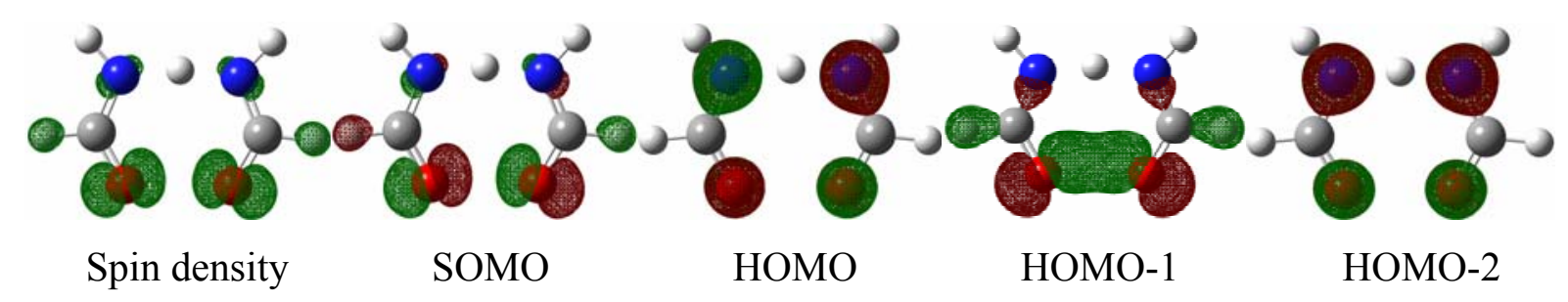

Figure S5. Total spin density surface (first) and four highest occupied molecular orbitals for FF $^{\text {ts }}$ obtained at the UB3LYP/6-311++G** level. The combination of SOMO and HOMO-1 contributes to a three-electron $\sigma$-bond between oxygen atoms, while HOMO and HOMO-2 are the $\pi$-type orbitals and their combination does not yield net bonding contribution to the O...O binding.
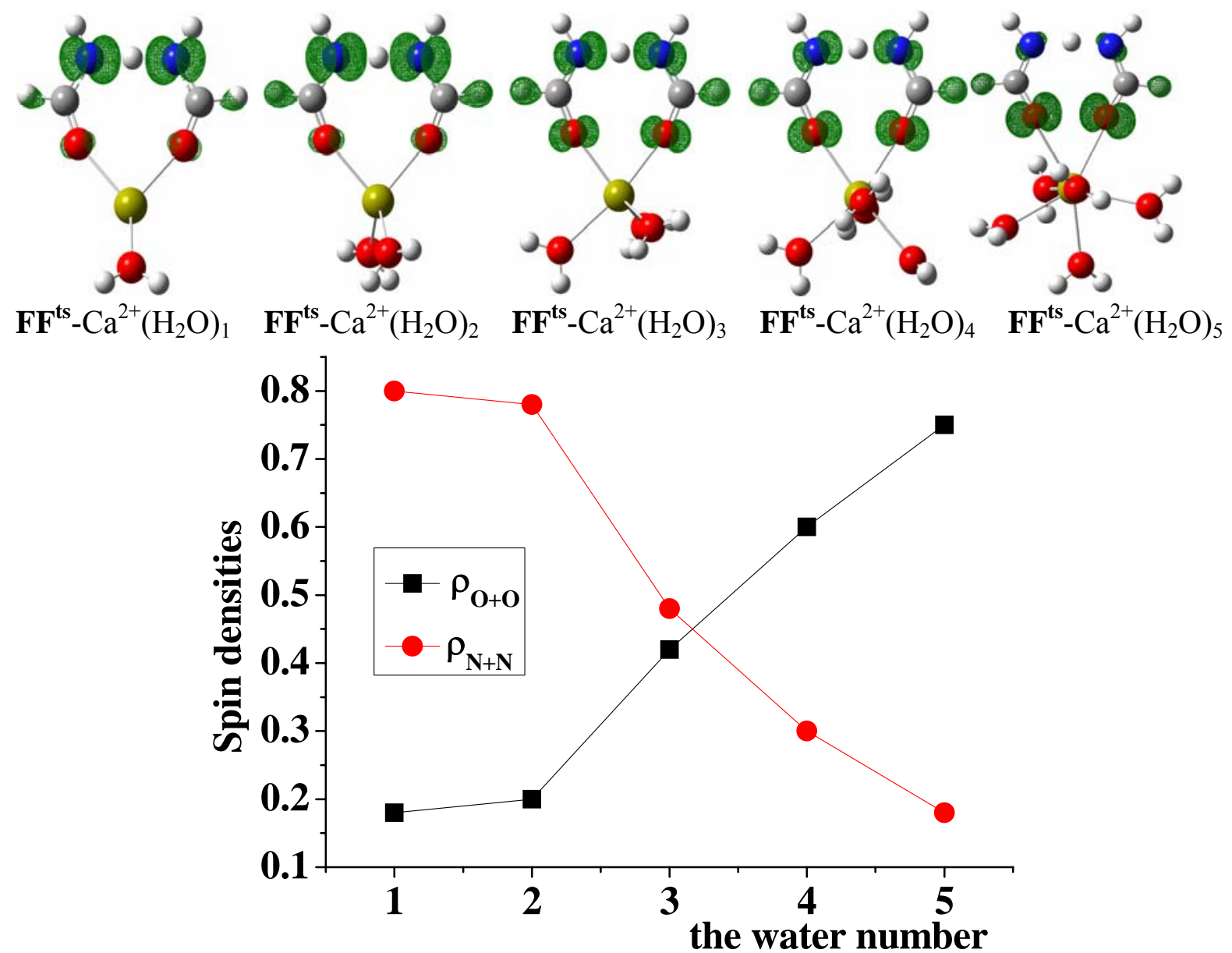

Figure S6. The total spin density surfaces and the dependence of the summed spin densities over two oxygen atoms $\left(\rho_{\mathrm{O}+\mathrm{O}}\right)$ and the summed spin densities over two nitrogen atoms $\left(\rho_{\mathrm{N}+\mathrm{N}}\right)$ on the water ligand numbers for $\mathbf{F F}^{\text {ts }}-\mathrm{Ca}^{2+}\left(\mathrm{H}_{2} \mathrm{O}\right)_{1}, \quad \mathbf{F F}^{\text {ts }}-\mathrm{Ca}^{2+}\left(\mathrm{H}_{2} \mathrm{O}\right)_{2}, \quad \mathbf{F F}^{\text {ts }}-\mathrm{Ca}^{2+}\left(\mathrm{H}_{2} \mathrm{O}\right)_{3}$, $\mathbf{F F}^{\text {ts }}-\mathrm{Ca}^{2+}\left(\mathrm{H}_{2} \mathrm{O}\right)_{4}$, and $\mathbf{F F}^{\text {ts }}-\mathrm{Ca}^{2+}\left(\mathrm{H}_{2} \mathrm{O}\right)_{5}$, respectively. 

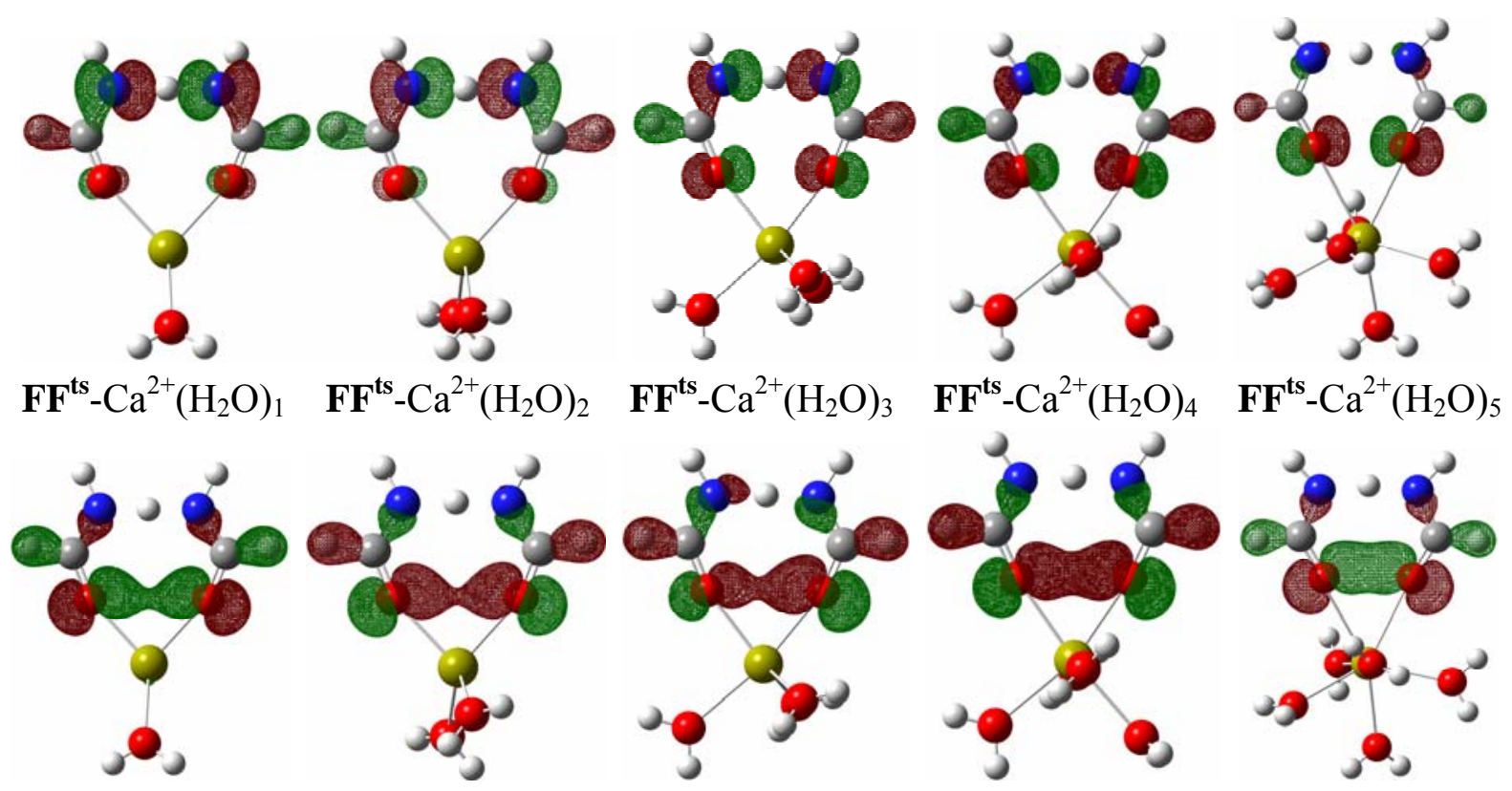

Figure S7. The singly occupied molecular orbitals (SOMO, upper) and doubly occupied molecular orbitals (lower) for five hydrated $\mathrm{Ca}^{2+}$ coupled transition states. The isospin value is $0.060 \mathrm{e} / \mathrm{au}^{3}$.
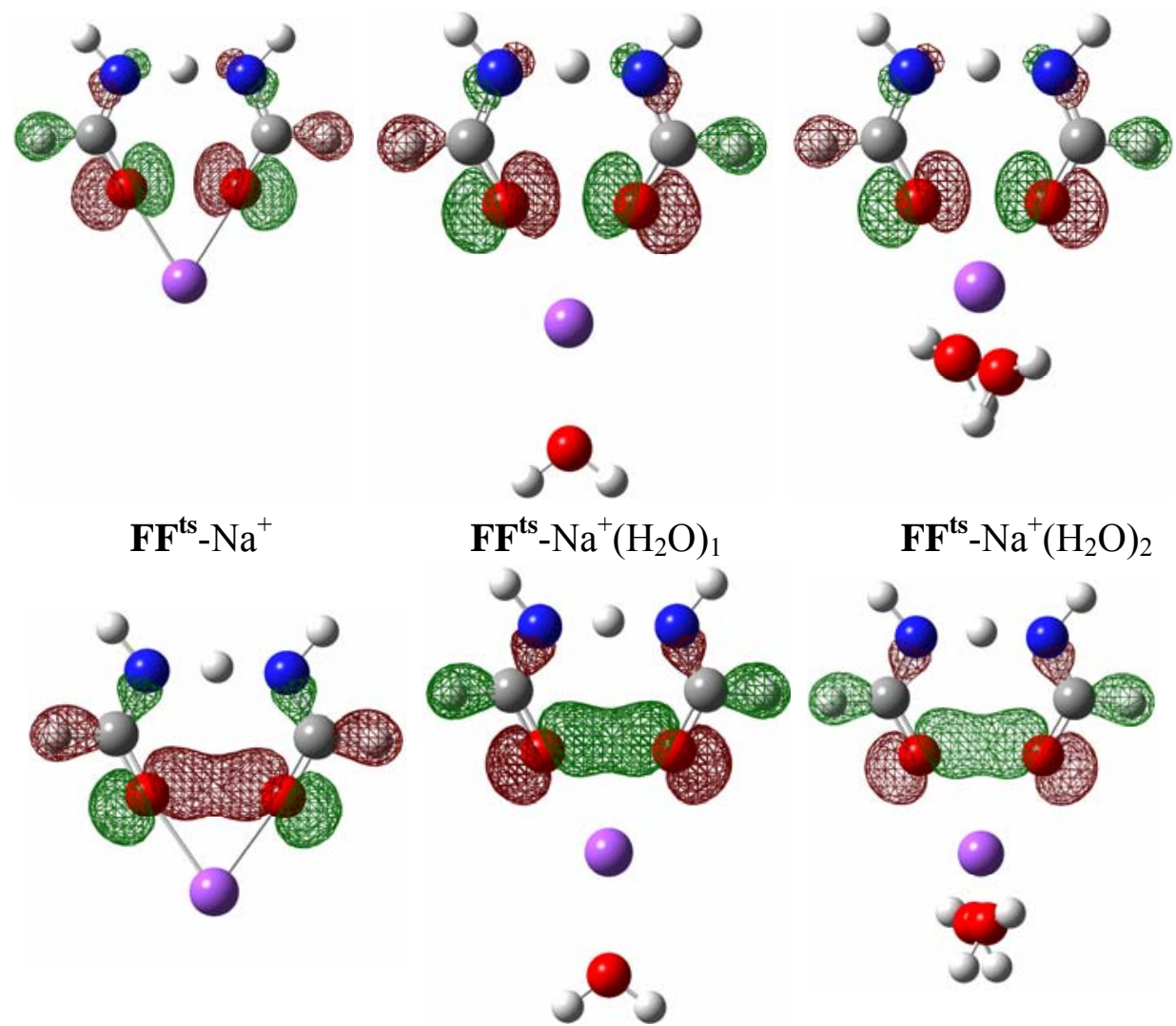

Figure S8. The singly occupied molecular orbitals (SOMO, upper) and doubly occupied molecular orbitals (lower) for three hydrated $\mathrm{Na}^{+}$coupled transition states. The isospin value is $0.060 \mathrm{e} / \mathrm{au}^{3}$. 

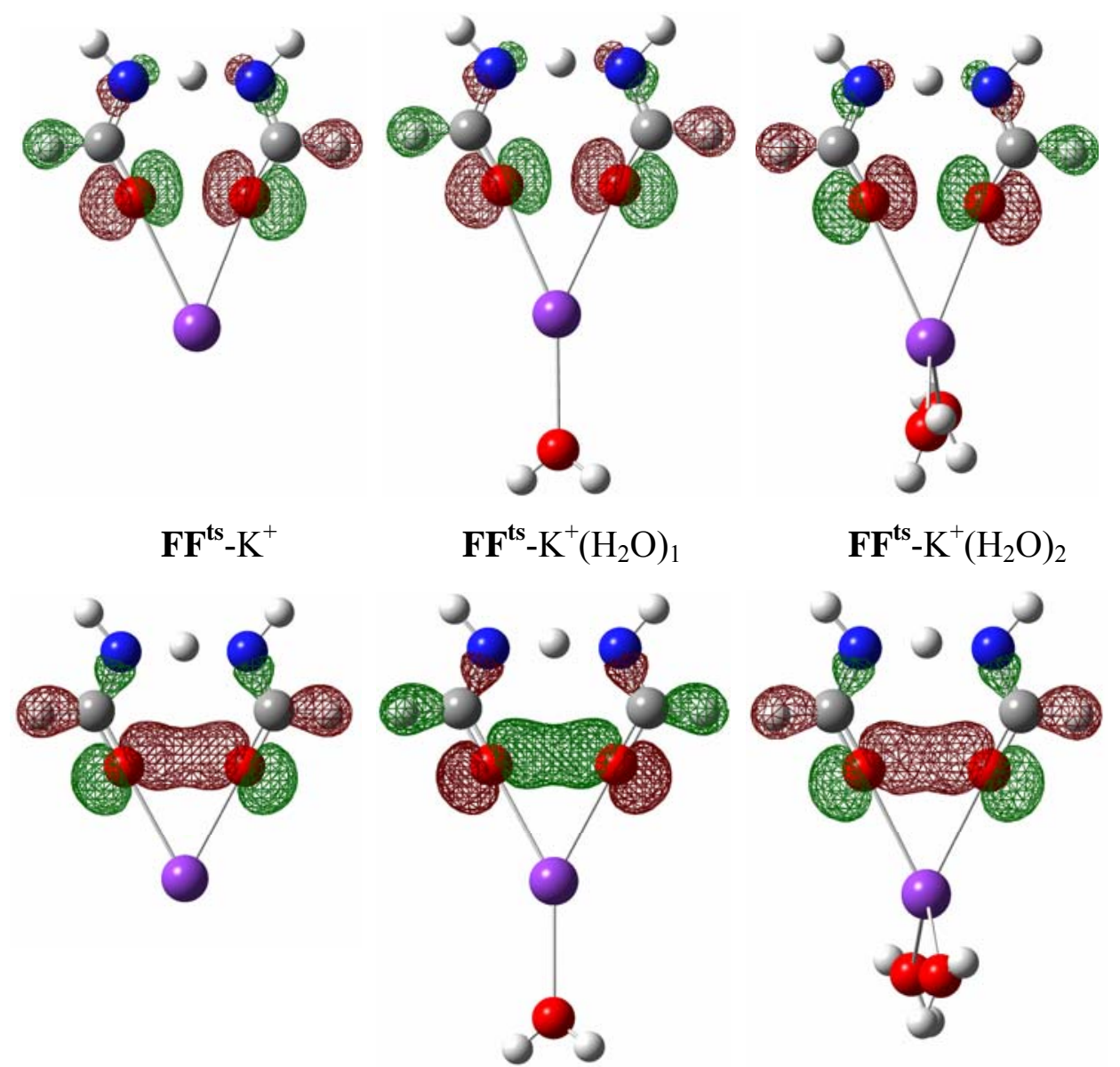

Figure S9. The singly occupied molecular orbitals (SOMO, upper) and doubly occupied molecular orbitals (lower) for three hydrated $\mathrm{K}^{+}$coupled transition states. The isospin value is $0.060 \mathrm{e} / \mathrm{au}^{3}$. 

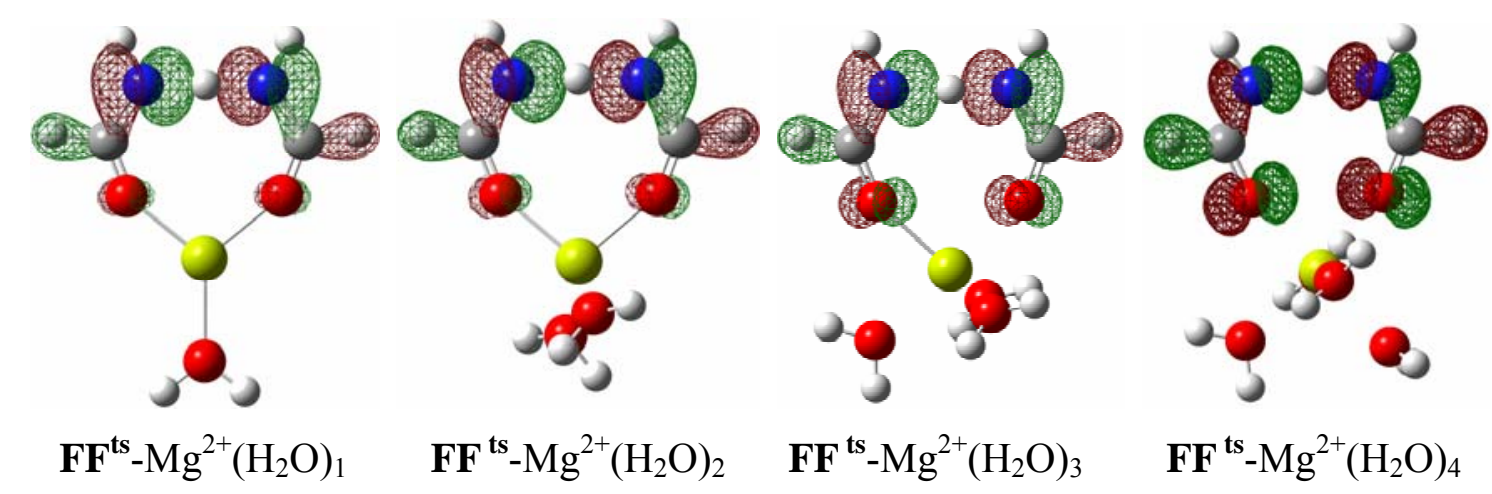

$\mathbf{F F}^{\text {ts }}-\mathrm{Mg}^{2+}\left(\mathrm{H}_{2} \mathrm{O}\right)_{3}$

$$
\text { FF }{ }^{\text {ts }}-\mathrm{Mg}^{2+}\left(\mathrm{H}_{2} \mathrm{O}\right)_{4}
$$
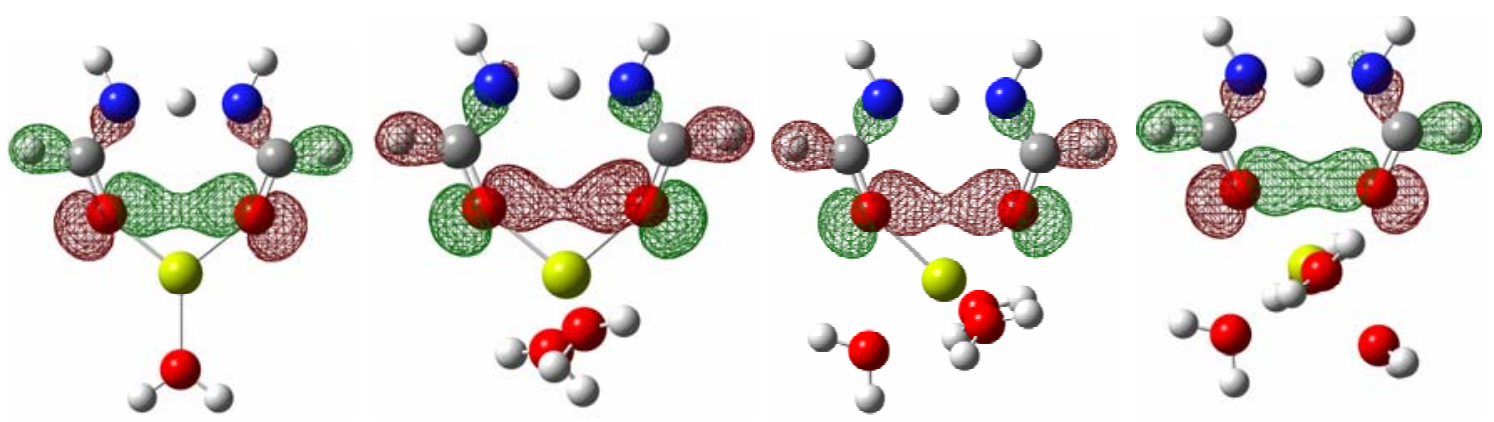

Figure S10. The singly occupied molecular orbitals (SOMO, upper) and doubly occupied molecular orbitals (lower) for four hydrated $\mathrm{Mg}^{2+}$ coupled transition states. The isospin value is $0.060 \mathrm{e} / \mathrm{au}^{3}$.

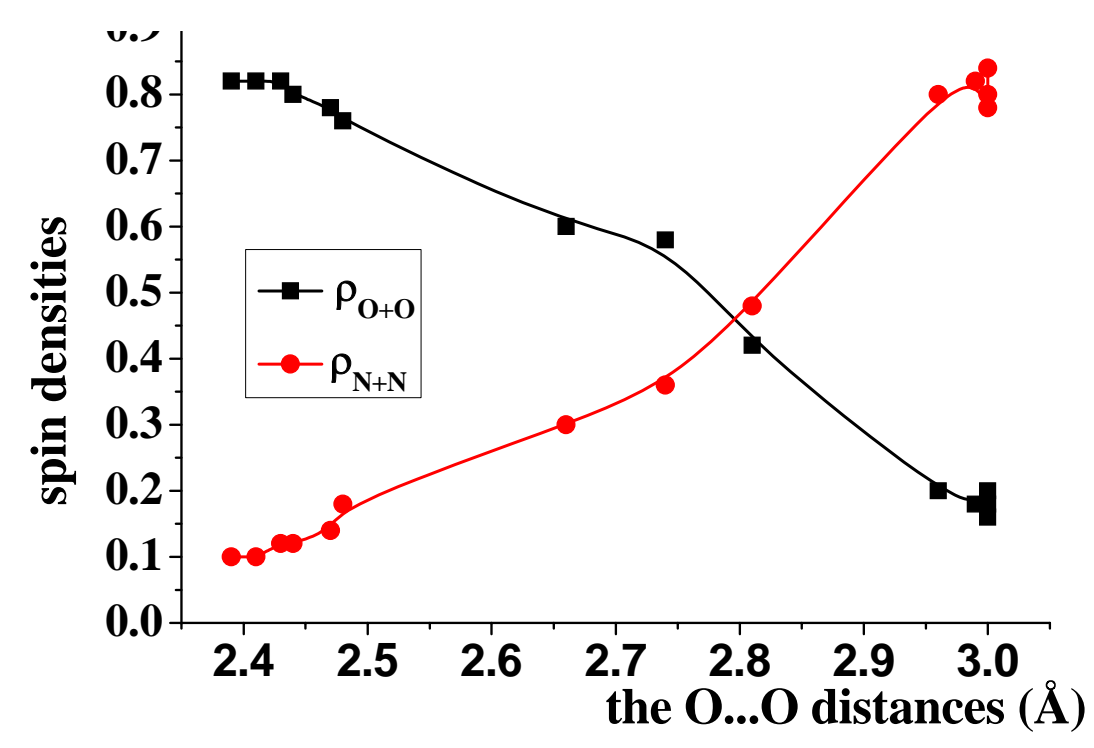

Figure S11. The relation between the summed spin densities over two oxygen atoms $\left(\rho_{\mathrm{O}+\mathrm{O}}\right)$ and two oxygen atoms $\left(\rho_{\mathrm{N}+\mathrm{N}}\right)$ and the $\mathrm{O} \cdots \mathrm{O}$ distances for all studied species. 


\section{Remarks for hydration effect on the FF complex--Molecular Dynamics Simulation}

MD routine: Classical Molecular Dynamics (Cerius2 version 4.6 Accelrys Inc., San Diego, CA) simulations was performed with COMPASS force field in a cubic cell (14.410 $\AA$ $\times 14.410 \AA \times 14.410 \AA$ ), corresponding to the density of $1.000 \mathrm{~g} / \mathrm{cm}^{-1}$. Periodic boundary conditions were applied in all three directions and the equations of motion were integrated within the canonical (NVT) ensemble. During the production run the Andersen (Andersen, H.C. J. Chem. Phys. 1980, 72, 2384-2393) method was used to control the temperature $(\mathrm{T}=298 \mathrm{~K})$. The integration time is $0.5 \mathrm{fs}$ to ensure constant temperature throughout the simulations, and the system was equilibrated for 500ps with the $\mathbf{F F}^{\mathbf{t s}}$ structure fixed.

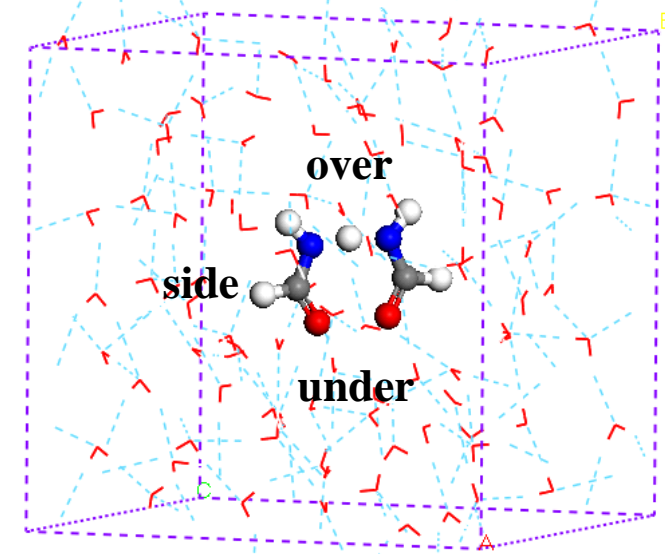

A

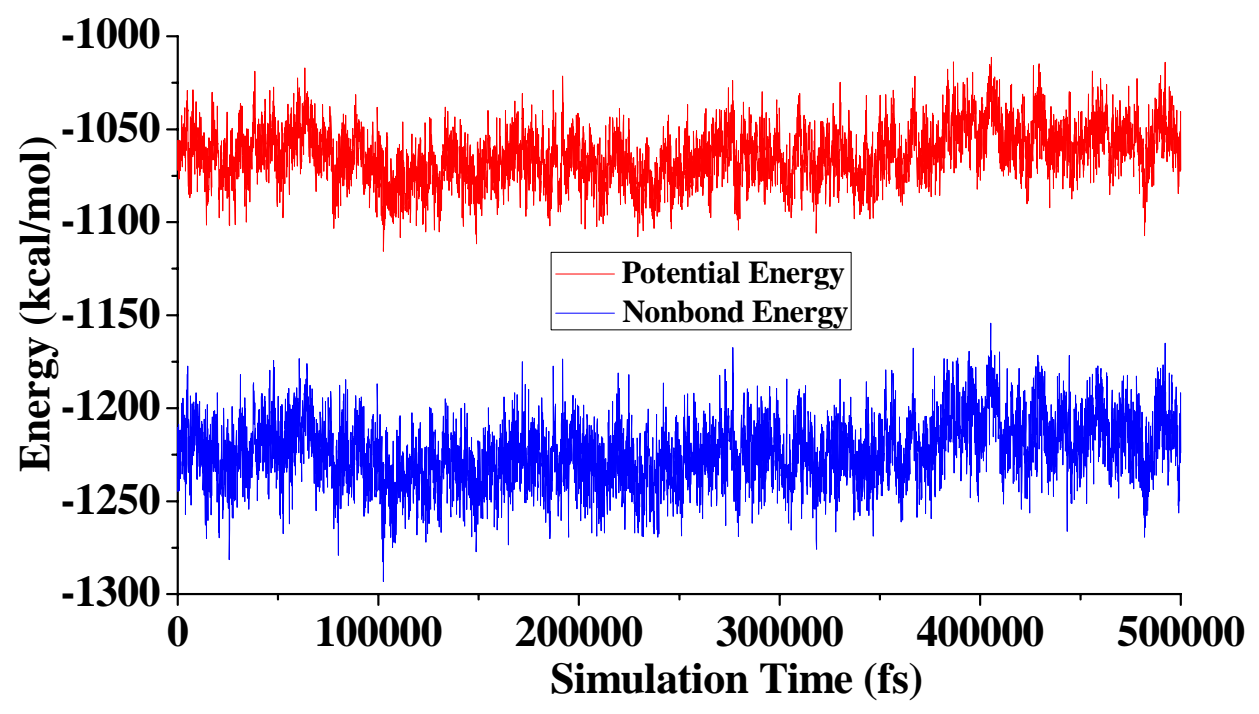

B

Figure S12. A) Snapshot of $\left(\mathbf{F F}^{\mathbf{t s}}+95 \mathrm{H}_{2} \mathrm{O}\right)$ system in which hydrogen bonds are shown in blue broken lines and waters in red. B) The MD energies of $\left(\mathbf{F F}^{\mathbf{t s}}+95 \mathrm{H}_{2} \mathrm{O}\right)$ system. 
Analysis: Figure S12 A shows a shoot picture of $\mathbf{F F}^{\text {ts }}$ in 95 water molecules and Figure $\mathrm{S} 12 \mathbf{B}$ is the plot of the total energies of this system as a function of time. Figure S12 B indicates that the solvated system had reached equilibrium of the NVT molecular dynamics simulation, which was an indication that the generated NVT molecular dynamics trajectories of these complexes were quite stable and this simulation was correct.

After minimizing the system, we found that no water molecule presents by the side of $\mathbf{F F}^{\text {ts }}$ as a donor or acceptor of hydrogen bond directly linking with $\mathbf{F F}^{\text {ts }}$. Water molecules can only form hydrogen bonding with $\mathbf{F F}^{\text {ts }}$ "over" (to two N-H) and "under" (to two O) FF $\mathbf{F}^{\mathbf{t s}}$. With this view in mind, we carried out molecular dynamics simulation for this minimized system for 500ps and analyzed the whole dynamics process. We also found same phenomenon as that in minimization. Clearly, these phenomena indicate that the "over" and "under" zones are hydrophilic, while the "side" zone is hydrophobic. Therefore, it was concluded that water molecules can affect FF PT/ET mechanism through the interaction with the N-H zone (over) and O...O zone (under). Figure S13 shows the statistics of the H-bond formed at different zones over 100 snapshot structures extracted from the molecular dynamics simulation. The number of the formed hydrogen bonds at the "under" and "over" zones is predominant in the total number of the formed hydrogen bonds of water molecules with $\mathbf{F F}{ }^{\mathbf{t s}}$. Our DFT calculations have also verified the above conclusion.

It should be noted that the study on the detailed PT/ET dynamics of this system is still in progress, using ab initio molecular dynamics simulation.

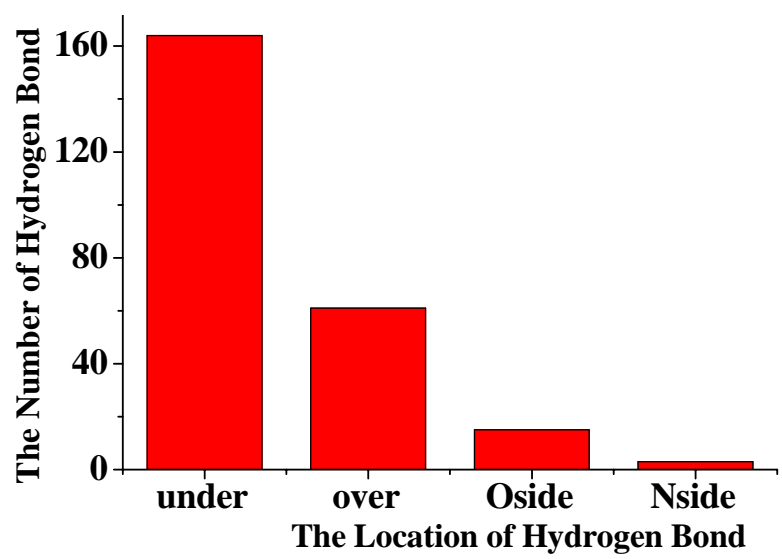

Figure S13. The statistics of hydrogen bonds of $\mathbf{F F}^{\text {ts }}$ over 100 snapshot structures of the 
trajectory file of the molecular dynamics simulation. For the H-bond geometry, maximum hydrogen-acceptor distance is $2.20 \AA$ and minimum donor-hydrogen-acceptor angle is $120.0^{\circ}$. The terms "under" and "over" are same as the definition in Figure S12A. O $\mathrm{O}_{\text {side }}$ denotes that $\mathbf{F F}^{\text {ts }}$ H-bonds to the water molecules through the "side" $\mathrm{O}$ zone, while $\mathrm{N}_{\text {side }}$ denotes that $\mathbf{F F}^{\text {ts }}$ H-bonds to the "side" $\mathrm{N}$ zone.

\section{Main Data}

Table S1. Natural Population Analysis (NPA) for the all reactant complexes optimized at the B3LYP/6-311++G** Level. Natural Atom charge $(\mathrm{Q})$ is in $e$. The last three columns are the $\mathrm{H}_{4} \cdots \mathrm{N}_{10}$ distances and the binding energies $\left(\Delta \mathrm{E}_{\mathrm{b}}\right)$ corrected with and without BSSE, respectively

\begin{tabular}{|c|c|c|c|c|c|c|c|}
\hline Species & $\mathrm{Q}_{\mathrm{O} 2}$ & Q & $\mathrm{Q}_{\mathrm{N} 3}$ & $\mathrm{Q}_{\mathrm{N} 10}$ & $\mathrm{~L}_{\mathrm{H} 4 \cdots \mathrm{N} 10}(\AA)$ & $\Delta \mathrm{E}_{\mathrm{b}}(\mathrm{kcal} / \mathrm{mol})$ & $\Delta \mathrm{E}_{\mathrm{b}}(\mathrm{kcal} / \mathrm{mol})_{\mathrm{BSSE}}$ \\
\hline FF & -0.501 & -0.316 & -0.795 & -0.791 & 1.80 & 0 & \\
\hline FF-Na ${ }^{+}$ & -0.763 & -0.643 & -0.757 & -0.367 & 2.59 & 55.9 & 55.0 \\
\hline FF-Na ${ }^{+}\left(\mathrm{H}_{2} \mathrm{O}\right)$ & -0.744 & -0.620 & -0.768 & -0.376 & 2.40 & 48.9 & 48.0 \\
\hline FF-Na ${ }^{+}\left(\mathrm{H}_{2} \mathrm{O}\right)_{2}$ & -0.723 & -0.598 & -0.776 & -0.381 & 2.32 & 41.3 & 40.3 \\
\hline FF- $\mathrm{Ca}^{2+}\left(\mathrm{H}_{2} \mathrm{O}\right)$ & -0.901 & -0.827 & -0.683 & -0.265 & 7.79 & 134.2 & 133.5 \\
\hline FF-Ca ${ }^{2+}\left(\mathrm{H}_{2} \mathrm{O}\right)_{2}$ & -0.871 & -0.791 & -0.695 & -0.281 & 7.76 & 116.2 & 115.3 \\
\hline FF-Ca ${ }^{2+}\left(\mathrm{H}_{2} \mathrm{O}\right)_{3}$ & -0.839 & -0.766 & -0.704 & -0.298 & 5.02 & 99.7 & 98.7 \\
\hline FF-Ca ${ }^{2+}\left(\mathrm{H}_{2} \mathrm{O}\right)_{4}$ & -0.814 & -0.693 & -0.733 & -0.352 & 2.50 & 84.2 & 83.1 \\
\hline FF-Ca ${ }^{2+}\left(\mathrm{H}_{2} \mathrm{O}\right)_{5}$ & -0.799 & -0.662 & -0.742 & -0.366 & 2.32 & 73.2 & 72.0 \\
\hline FF- $\mathrm{Mg}^{2+}\left(\mathrm{H}_{2} \mathrm{O}\right)$ & -0.929 & -0.869 & -0.672 & -0.247 & 7.85 & 176.1 & 174.5 \\
\hline FF- $\mathrm{Mg}^{2+}\left(\mathrm{H}_{2} \mathrm{O}\right)_{2}$ & -0.893 & -0.813 & -0.686 & -0.275 & 5.55 & 145.7 & 144.0 \\
\hline FF- $\mathrm{Mg}^{2+}\left(\mathrm{H}_{2} \mathrm{O}\right)_{3}$ & -0.853 & -0.739 & -0.714 & -0.339 & 2.52 & 118.2 & 116.2 \\
\hline FF- $\mathrm{Mg}^{2+}\left(\mathrm{H}_{2} \mathrm{O}\right)_{4}$ & -0.828 & -0.697 & -0.724 & -0.354 & 2.53 & 97.3 & 94.9 \\
\hline FF-K ${ }^{+}$ & -0.742 & -0.615 & -0.767 & -0.379 & 2.36 & 42.2 & 41.9 \\
\hline FF- $\mathrm{K}^{+}\left(\mathrm{H}_{2} \mathrm{O}\right)$ & -0.662 & -0.438 & -0.747 & -0.708 & 1.85 & 31.1 & 30.8 \\
\hline FF- $\mathrm{K}^{+}\left(\mathrm{H}_{2} \mathrm{O}\right)_{2}$ & -0.619 & -0.435 & -0.747 & -0.727 & 1.82 & 27.0 & 26.7 \\
\hline FF- $\left(\mathrm{H}_{2} \mathrm{O}\right)_{1}$ & & & & & & 5.8 & 5.3 \\
\hline FF- $\left(\mathrm{H}_{2} \mathrm{O}\right)_{2}$ & & & & & & 9.8 & 9.0 \\
\hline FF- $\left(\mathrm{H}_{2} \mathrm{O}\right)_{3}$ & & & & & & 9.0 & 8.3 \\
\hline $\mathbf{F F}-\left(\mathrm{H}_{2} \mathrm{O}\right)_{4}$ & & & & & & 9.1 & 8.4 \\
\hline FF- $\left(\mathrm{H}_{2} \mathrm{O}\right)_{5}$ & & & & & & 11.0 & 10.1 \\
\hline FF- $\left(\mathrm{H}_{2} \mathrm{O}\right)_{6}$ & & & & & & 15.2 & 14.0 \\
\hline FF- $\left(\mathrm{H}_{2} \mathrm{O}\right)_{4}-\mathrm{N}-\left(\mathrm{H}_{2} \mathrm{O}\right)_{2}$ & & & & & & 19.9 & 17.7 \\
\hline FF- $\left(\mathrm{H}_{2} \mathrm{O}\right)_{2}-\mathrm{N}-\left(\mathrm{H}_{2} \mathrm{O}\right)_{4}$ & & & & & & 15.9 & 14.5 \\
\hline
\end{tabular}


Table S2. The barrier heights $\left(\Delta \mathrm{E}_{\mathrm{a}}\right.$, the electronic energies) without zero-point vibrational energy, and the activation enthalpies $\left(\Delta \mathrm{H}_{\mathrm{a}}\right)$. All energies are in $\mathrm{kcal} / \mathrm{mol}$. Selected geometrical parameters for the transition states of the FF- $\mathbf{M}^{\mathrm{z}+}\left(\mathrm{H}_{2} \mathrm{O}\right)_{\mathrm{n}}$ complexes together with spin densities on oxygens $\left(\rho_{\text {spin }}^{\mathrm{O}}\right)$ and nitrogen $\left(\rho^{\mathrm{N}}{ }_{\text {spin }}\right)$. All values are obtained at the UB3LYP/6-311++G** level.

\begin{tabular}{|c|c|c|c|c|c|c|c|}
\hline Species & $\begin{array}{l}\Delta \mathrm{E}_{\mathrm{a}} \\
(\mathrm{kcal} / \mathrm{mol})\end{array}$ & $\begin{array}{l}\Delta \mathrm{H}_{\mathrm{a}} \\
(\mathrm{kcal} / \mathrm{mol})\end{array}$ & $\begin{array}{l}R_{\mathrm{O}-\mathrm{O}} \\
(\AA)\end{array}$ & $\begin{array}{l}R_{\mathrm{N}-\mathrm{H}-\mathrm{N}} \\
(\AA)\end{array}$ & $\begin{array}{l}A_{\mathrm{NHN}} \\
\left(^{\circ}\right)\end{array}$ & $\rho_{\text {spin }}^{O}$ & $\rho_{\text {spin }}^{\mathrm{N}}$ \\
\hline $\mathbf{F F}^{\text {ts }}$ & 4.3 & 1.4 & 2.29 & 2.54 & 169.0 & 0.44 & 0.03 \\
\hline $\mathbf{F F}^{\mathbf{t s}}-\mathrm{Ca}^{2+}\left(\mathrm{H}_{2} \mathrm{O}\right)_{1}$ & 25.9 & 21.7 & 2.99 & 2.50 & 165.6 & 0.09 & 0.40 \\
\hline $\mathbf{F F}^{\mathbf{t s}}-\mathrm{Ca}^{2+}\left(\mathrm{H}_{2} \mathrm{O}\right)_{2}$ & 22.5 & 18.3 & 3.00 & 2.50 & 165.9 & 0.10 & 0.39 \\
\hline $\mathbf{F F}^{\mathbf{t s}}-\mathrm{Ca}^{2+}\left(\mathrm{H}_{2} \mathrm{O}\right)_{3}$ & 18.5 & 13.8 & 2.81 & 2.52 & 176.8 & 0.21 & 0.24 \\
\hline $\mathbf{F F}^{\mathbf{t s}}-\mathrm{Ca}^{2+}\left(\mathrm{H}_{2} \mathrm{O}\right)_{4}$ & 15.6 & 11.5 & 2.66 & 2.54 & 174.2 & $0.29 / 0.31$ & 0.15 \\
\hline $\mathbf{F F}^{\mathbf{t s}}-\mathrm{Ca}^{2+}\left(\mathrm{H}_{2} \mathrm{O}\right)_{5}$ & 13.4 & 10.7 & 2.48 & 2.54 & 171.8 & $0.38 / 0.37$ & 0.09 \\
\hline $\mathbf{F F}^{\mathbf{t s}}-\mathrm{Mg}^{2+}\left(\mathrm{H}_{2} \mathrm{O}\right)_{1}$ & 28.1 & 23.9 & 3.01 & 2.50 & 164.8 & 0.08 & 0.42 \\
\hline $\mathbf{F F}^{\mathbf{t s}}-\mathrm{Mg}^{2+}\left(\mathrm{H}_{2} \mathrm{O}\right)_{2}$ & 22.4 & 18.4 & 2.99 & 2.49 & 164.6 & 0.09 & 0.41 \\
\hline $\mathbf{F F}^{\mathbf{t s}}-\mathrm{Mg}^{2+}\left(\mathrm{H}_{2} \mathrm{O}\right)_{3}$ & 16.6 & 12.4 & 2.96 & 2.48 & 164.1 & 0.10 & 0.40 \\
\hline $\mathbf{F F}^{\mathbf{t s}}-\mathrm{Mg}^{2+}\left(\mathrm{H}_{2} \mathrm{O}\right)_{4}$ & 16.3 & 11.6 & 2.74 & 2.52 & 175.3 & $0.27 / 0.29$ & 0.18 \\
\hline $\mathbf{F F}^{\mathbf{t s}}-\mathrm{K}^{+}$ & 11.2 & 8.4 & 2.43 & 2.55 & 171.2 & 0.41 & 0.06 \\
\hline $\mathbf{F F}^{\mathbf{t s}}-\mathrm{K}^{+}\left(\mathrm{H}_{2} \mathrm{O}\right)_{1}$ & 4.4 & 2.1 & 2.41 & 2.55 & 170.9 & 0.41 & 0.05 \\
\hline $\mathbf{F F}^{\mathbf{t s}}-\mathrm{K}^{+}\left(\mathrm{H}_{2} \mathrm{O}\right)_{2}$ & 4.3 & 1.7 & 2.39 & 2.55 & 170.5 & 0.42 & 0.05 \\
\hline $\mathbf{F F}^{\mathbf{t s}}-\mathrm{Na}^{+}$ & 13.1 & 10.2 & 2.47 & 2.55 & 171.8 & 0.39 & 0.07 \\
\hline $\mathbf{F F}^{\mathbf{t s}}-\mathrm{Na}^{+}\left(\mathrm{H}_{2} \mathrm{O}\right)_{1}$ & 11.8 & 9.0 & 2.44 & 2.55 & 171.3 & 0.40 & 0.06 \\
\hline $\mathbf{F F}^{\mathbf{t s}}-\mathrm{Na}^{+}\left(\mathrm{H}_{2} \mathrm{O}\right)_{2}$ & 10.5 & 7.8 & 2.41 & 2.55 & 170.8 & 0.41 & 0.05 \\
\hline $\mathbf{F F}^{\text {ts }}-\left(\mathrm{H}_{2} \mathrm{O}\right)_{1}$ & 4.5 & 1.7 & 2.30 & 2.54 & 169.0 & $0.46 / 0.40$ & 0.03 \\
\hline $\mathbf{F F}^{\mathbf{t s}}-\left(\mathrm{H}_{2} \mathrm{O}\right)_{2}$ & 4.8 & 1.9 & 2.30 & 2.54 & 169.2 & $0.45 / 0.42$ & 0.03 \\
\hline $\mathbf{F F}^{\text {ts }}-\left(\mathrm{H}_{2} \mathrm{O}\right)_{3}$ & 4.3 & 1.5 & 2.30 & 2.54 & 169.3 & 0.43 & 0.03 \\
\hline $\mathbf{F F}^{\text {ts }}-\left(\mathrm{H}_{2} \mathrm{O}\right)_{4}$ & 4.4 & 1.5 & 2.29 & 2.54 & 169.2 & 0.43 & 0.03 \\
\hline $\mathbf{F F}^{\text {ts }}-\left(\mathrm{H}_{2} \mathrm{O}\right)_{5}$ & 4.4 & 1.6 & 2.30 & 2.55 & 169.3 & 0.42 & 0.03 \\
\hline $\mathbf{F F}^{\text {ts }}-\left(\mathrm{H}_{2} \mathrm{O}\right)_{6}$ & 4.1 & 1.3 & 2.34 & 2.54 & 169.9 & 0.41 & 0.04 \\
\hline $\mathbf{F F}^{\text {ts }}-\mathrm{N}-\left(\mathrm{H}_{2} \mathrm{O}\right)_{2}$ & 4.7 & 1.8 & 2.30 & 2.55 & 169.5 & 0.43 & 0.03 \\
\hline $\mathbf{F F}^{\mathbf{t s}}-\left(\mathrm{H}_{2} \mathrm{O}\right)_{4}-\mathrm{N}-\left(\mathrm{H}_{2} \mathrm{O}\right)_{2}$ & 4.8 & 1.9 & 2.30 & 2.55 & 169.7 & 0.42 & 0.04 \\
\hline $\mathbf{F F}^{\text {ts }}-\left(\mathrm{H}_{2} \mathrm{O}\right)_{4}-\mathrm{N}-\left(\mathrm{H}_{2} \mathrm{O}\right)_{4}$ & 4.4 & 1.3 & 2.32 & 2.55 & 170.7 & 0.42 & 0.04 \\
\hline
\end{tabular}


Table S3. Some main parameters of formamide (F) and N-dehydrogened formamide radical (Fr) obtained from different methods and other reports

\begin{tabular}{|c|c|c|c|c|c|c|c|c|c|c|}
\hline & & B3LYP ${ }^{a}$ & $\mathrm{~B}^{2} \mathrm{LYP}^{\mathrm{b}}$ & MPW1PW91 ${ }^{\mathrm{b}}$ & PBE1PBE ${ }^{b}$ & B3PW91 ${ }^{\mathrm{b}}$ & $\mathrm{MP}^{2}{ }^{\mathrm{b}}$ & BHandHLYP $^{\mathrm{b}}$ & $\operatorname{Exp}^{c}$ & ${ }_{B 3} \mathrm{LYP}^{\mathrm{d}}$ \\
\hline \multirow{9}{*}{$\mathrm{F}$} & $R_{\mathrm{C}=\mathrm{O}}$ & 1.21 & 1.22 & 1.21 & 1.21 & 1.22 & 1.23 & 1.20 & 1.22 & 1.22 \\
\hline & $R_{\mathrm{C}-\mathrm{N}}$ & 1.36 & 1.36 & 1.36 & 1.36 & 1.36 & 1.37 & 1.35 & 1.35 & 1.36 \\
\hline & $R_{\mathrm{N}-\mathrm{Hl}}$ & 1.01 & 1.01 & 1.01 & 1.01 & 1.01 & 1.01 & 1.00 & 1.00 & 1.01 \\
\hline & $R_{\mathrm{N}-\mathrm{H} 2}$ & 1.01 & 1.01 & 1.01 & 1.01 & 1.01 & 1.01 & 1.00 & 1.00 & 1.01 \\
\hline & $R_{\mathrm{C}-\mathrm{H3}}$ & 1.11 & 1.11 & 1.11 & 1.11 & 1.11 & 1.11 & 1.10 & 1.09 & 1.11 \\
\hline & $A_{0-\mathrm{C}-\mathrm{N}}$ & 124.8 & 124.7 & 124.8 & 124.8 & 124.8 & 124.6 & 124.7 & 124.7 & 124.7 \\
\hline & $A_{\mathrm{O}-\mathrm{C}-\mathrm{H}}$ & 122.6 & 122.7 & 122.6 & 122.6 & 122.7 & 122.8 & 122.4 & 122.6 & 122.7 \\
\hline & $A_{\mathrm{C}-\mathrm{N}-\mathrm{H}}$ & 121.3 & 121.3 & 121.3 & 121.3 & 121.3 & 121.1 & 121.3 & 120.0 & 121.0 \\
\hline & $D_{\text {O-C-N-H }}$ & 180.0 & 180.0 & 180.0 & 180.0 & 180.0 & 180.0 & 180.0 & 180.0 & 180.0 \\
\hline \multirow{8}{*}{$\mathrm{Fr}$} & $R_{\mathrm{C}=\mathrm{O}}$ & 1.20 & 1.21 & 1.21 & 1.21 & 1.21 & 1.22 & & & \\
\hline & $R_{\mathrm{C}-\mathrm{N}}$ & 1.39 & 1.39 & 1.39 & 1.39 & 1.40 & 1.41 & & & \\
\hline & $R_{\mathrm{N}-\mathrm{H} 2}$ & 1.03 & 1.03 & 1.03 & 1.03 & 1.03 & 1.03 & & & \\
\hline & $R_{\mathrm{C}-\mathrm{H} 3}$ & 1.12 & 1.12 & 1.12 & 1.12 & 1.12 & 1.11 & & & \\
\hline & $A_{0-\mathrm{C}-\mathrm{N}}$ & 125.6 & 125.2 & 125.2 & 125.4 & 125.4 & 124.4 & & & \\
\hline & $A_{\mathrm{O}-\mathrm{C}-\mathrm{H}}$ & 122.7 & 122.7 & 122.8 & 122.8 & 122.8 & 123.1 & & & \\
\hline & $A_{\mathrm{C}-\mathrm{N}-\mathrm{H}}$ & 111.1 & 111.1 & 111.0 & 111.0 & 111.7 & 109.9 & & & \\
\hline & $D_{\text {O-C-N-H }}$ & 113.5 & 115.8 & 107.0 & 109.2 & 111.4 & 91.5 & & & \\
\hline
\end{tabular}

Notes: a) obtained using $6-311++\mathrm{G}^{* *}$ basis set; b) obtained using aug-cc-PVDZ basis set. The bond lengths are in angstrom $(\AA)$ and the angles and dihedral angles are in degree $\left({ }^{\circ}\right)$; c) Sugisaki, R.; Tanaka, T.; Hirota, E. J .Mol. Spectrosc. 1974, 49, 294; d) Cabaleiro-Lago, E. M.; Otero, J. R. J. Chem. Phys. 2002, $117,1621$.

Table S4. Comparisons of the barrier heights $\left(\Delta \mathrm{E}_{\mathrm{a}}\right)$ without zero-point energies, and the activation enthalpies $\left(\Delta H_{a}\right)$, selected geometrical parameters, spin densities on oxygens $\left(\rho^{O}\right.$ spin $)$ and nitrogen $\left(\rho^{\mathrm{N}}{ }_{\text {spin }}\right)$ for the $\mathbf{F F}^{\text {ts }}$ transition state, and the binding energies $\left(\Delta \mathrm{E}_{\mathrm{b}}\right)$ for $\mathbf{F F}$, obtained using different methods. All energies are in $\mathrm{kcal} / \mathrm{mol}$.

\begin{tabular}{llllllllll}
\hline FF $^{\text {ts }}$ & $\begin{array}{l}R_{\mathrm{O}-\mathrm{O}} \\
(\AA)\end{array}$ & $\begin{array}{l}R_{\mathrm{N} \cdots \mathrm{H} \cdots \mathrm{N}} \\
(\AA)\end{array}$ & $\begin{array}{c}R_{\mathrm{N} \cdots \mathrm{H}} \\
(\AA)\end{array}$ & $\begin{array}{l}A_{\mathrm{NHN}} \\
\left({ }^{\circ}\right)\end{array}$ & $\rho_{\text {spin }}^{\mathrm{O}}$ & $\rho_{\text {spin }}^{\mathrm{N}}$ & $\Delta \mathrm{E}_{\mathrm{a}}$ & $\Delta \mathrm{E}_{\mathrm{b}}$ & $\Delta \mathrm{H}_{\mathrm{a}}$ \\
\hline B3LYP/6-311++G** & 2.29 & 2.54 & 1.28 & 169.0 & 0.44 & 0.03 & 4.3 & 2.8 & 1.4 \\
MP2/6-311++G** & & & & & & & 4.0 & & \\
B3LYP/aug-cc-PVDZ & 2.29 & 2.55 & 1.28 & 169.1 & 0.46 & 0.03 & 3.9 & 3.5 & 1.0 \\
MPW1PW91/aug-cc-PVDZ & 2.25 & 2.53 & 1.27 & 168.7 & 0.45 & 0.03 & $3.5(3.9)$ & 2.0 & 1.0 \\
B3PW91/aug-cc-PVDZ & 2.28 & 2.54 & 1.27 & 169.0 & 0.44 & 0.03 & $3.0(3.3)$ & 2.8 & 0.6 \\
PBE1PBE/aug-cc-PVDZ & 2.25 & 2.53 & 1.27 & 168.6 & 0.44 & 0.03 & $3.3(3.6)$ & 3.4 & 0.8 \\
& & & & & & & & &
\end{tabular}

\footnotetext{
${ }^{a}$ at the B3LYP/6-311++ ${ }^{* *}$ geometry.
} 


\section{Standard orientations of all structures}

Table S5: B3LYP/6-311++G** optimized structure for FF. Standard orientation:

\begin{tabular}{|c|c|c|c|c|}
\hline \multirow{2}{*}{$\begin{array}{l}\text { Center } \\
\text { Number }\end{array}$} & \multirow{2}{*}{$\begin{array}{l}\text { Atomic } \\
\text { Number }\end{array}$} & \multicolumn{3}{|c|}{ Coordinates (Angstroms) } \\
\hline & & $\mathrm{X}$ & $\mathrm{Y}$ & Z \\
\hline 1 & 6 & -1.847915 & -0.163873 & -0.000114 \\
\hline 2 & 8 & -1.141705 & -1.170862 & 0.000841 \\
\hline 3 & 7 & -1.426425 & 1.101629 & -0.000516 \\
\hline 4 & 1 & -2.945395 & -0.284432 & -0.000217 \\
\hline 5 & 1 & -0.402650 & 1.295983 & -0.000191 \\
\hline 6 & 1 & -2.101849 & 1.848881 & -0.000589 \\
\hline 7 & 1 & 2.934169 & -0.265729 & 0.000057 \\
\hline 8 & 6 & 1.841821 & -0.061843 & -0.000179 \\
\hline 9 & 8 & 1.223898 & -1.176652 & -0.000687 \\
\hline 10 & 7 & 1.390094 & 1.144225 & 0.000578 \\
\hline 11 & 1 & 2.149061 & 1.818729 & 0.001032 \\
\hline
\end{tabular}

Energy: -339.2186701a.u.

Enthalpy: -339.131026a.u.

Table S6: B3LYP/6-311++G** optimized structure for $\mathbf{F F}^{\text {ts }}$. Standard orientation:

\begin{tabular}{|c|c|c|c|c|}
\hline \multirow{2}{*}{$\begin{array}{l}\text { Center } \\
\text { Number }\end{array}$} & \multirow{2}{*}{$\begin{array}{l}\text { Atomic } \\
\text { Number }\end{array}$} & \multicolumn{3}{|c|}{ Coordinates (Angstroms) } \\
\hline & & $\mathrm{X}$ & Y & Z \\
\hline 1 & 6 & -0.000004 & 1.767262 & -0.096770 \\
\hline 2 & 8 & 0.000174 & 1.146306 & -1.185843 \\
\hline 3 & 7 & -0.000174 & 1.271855 & 1.114409 \\
\hline 4 & 1 & -0.000018 & 2.866890 & -0.209182 \\
\hline 5 & 1 & 0.000000 & 0.000000 & 1.237056 \\
\hline 6 & 1 & -0.000285 & 1.959049 & 1.857149 \\
\hline 7 & 1 & 0.000018 & -2.866890 & -0.209182 \\
\hline 8 & 6 & 0.000004 & -1.767262 & -0.096770 \\
\hline 9 & 8 & -0.000174 & -1.146306 & -1.185843 \\
\hline 10 & 7 & 0.000174 & -1.271855 & 1.114409 \\
\hline 11 & 1 & 0.000285 & -1.959049 & 1.857149 \\
\hline
\end{tabular}

Energy: -339.2118599a.u.

Enthalpy: -339.128751a.u.

Imaginary Frequency: $-1300.0 \mathrm{~cm}^{-1}$ 
Table S7: B3LYP/6-311++G ${ }^{* *}$ optimized structure for FF-Ca ${ }^{2+}\left(\mathrm{H}_{2} \mathrm{O}\right)_{1}$. Standard orientation:

\begin{tabular}{|c|c|c|c|c|}
\hline \multirow{2}{*}{$\begin{array}{l}\text { Center } \\
\text { Number }\end{array}$} & \multirow{2}{*}{$\begin{array}{l}\text { Atomic } \\
\text { Number }\end{array}$} & \multicolumn{3}{|c|}{ Coordinates (Angstroms) } \\
\hline & & $\mathrm{X}$ & $\mathrm{Y}$ & $\mathrm{Z}$ \\
\hline 1 & 6 & -2.939222 & -0.940033 & -0.477587 \\
\hline 2 & 8 & -1.879642 & -0.334715 & -0.159199 \\
\hline 3 & 7 & -3.856097 & -1.317426 & 0.380971 \\
\hline 4 & 1 & -3.139523 & -1.180797 & -1.525236 \\
\hline 5 & 1 & -3.761116 & -1.140532 & 1.374939 \\
\hline 6 & 1 & -4.689229 & -1.801197 & 0.066405 \\
\hline 7 & 1 & 3.596156 & -0.997958 & 1.411925 \\
\hline 8 & 6 & 3.127150 & -0.915597 & 0.411548 \\
\hline 9 & 8 & 2.019191 & -0.356803 & 0.275073 \\
\hline 10 & 7 & 3.780670 & -1.580949 & -0.517586 \\
\hline 11 & 1 & 4.773578 & -1.486215 & -0.747909 \\
\hline 12 & 8 & 0.037951 & 2.972830 & -0.075042 \\
\hline 13 & 1 & -0.732505 & 3.543389 & -0.220283 \\
\hline 14 & 1 & 0.786804 & 3.582266 & 0.013420 \\
\hline 15 & 20 & 0.057313 & 0.632647 & 0.032631 \\
\hline
\end{tabular}

Energy: -1092.8891582a.u.

Enthalpy: --1092.770139a.u.

Table S8: B3LYP/6-311++G** optimized structure for $\mathbf{F F}^{\text {ts }}-\mathrm{Ca}^{2+}\left(\mathrm{H}_{2} \mathrm{O}\right)_{1}$. Standard orientation:

\begin{tabular}{ccccc} 
Center & Atomic & \multicolumn{3}{c}{ Coordinates (Angstroms) } \\
Number & Number & X & Y & $Z$ \\
\hline 1 & 6 & -1.323732 & -0.279311 & -1.944105 \\
2 & 8 & -1.332634 & -0.060310 & -0.717056 \\
3 & 7 & -0.254442 & -0.470490 & -2.697125 \\
4 & 1 & -2.282547 & -0.398700 & -2.479433 \\
5 & 1 & 0.910329 & -0.467899 & -2.219346 \\
6 & 1 & -0.344495 & -0.430309 & -3.712747 \\
7 & 1 & 3.358596 & 0.361656 & -0.326017 \\
8 & 6 & 2.290996 & 0.207923 & -0.564246 \\
9 & 8 & 1.441764 & 0.313629 & 0.342019 \\
10 & 7 & 2.061075 & -0.158364 & -1.813224 \\
11 & 1 & 2.782667 & -0.008771 & -2.519015 \\
12 & 8 & -1.236460 & -0.405775 & 3.381417
\end{tabular}




$\begin{array}{rrrrr}13 & 1 & -2.120614 & -0.715678 & 3.634717 \\ 14 & 1 & -0.695890 & -0.523940 & 4.178755 \\ 15 & 20 & -0.551971 & 0.411680 & 1.300730\end{array}$

Energy: -1092.8479019a.u.

Enthalpy: -1092.735615a.u.

Imaginary Frequency: $-1779.445 \mathrm{~cm}^{-1}$

Table S9: B3LYP/6-311++G** optimized structure for FF-Ca ${ }^{2+}\left(\mathrm{H}_{2} \mathrm{O}\right)_{2}$. Standard orientation:

\begin{tabular}{|c|c|c|c|c|}
\hline \multirow{2}{*}{$\begin{array}{l}\text { Center } \\
\text { Number }\end{array}$} & \multirow{2}{*}{$\begin{array}{l}\text { Atomic } \\
\text { Number }\end{array}$} & \multicolumn{3}{|c|}{ Coordinates (Angstroms) } \\
\hline & & $\mathrm{X}$ & $\mathrm{Y}$ & Z \\
\hline 1 & 6 & -3.084096 & -0.800866 & -0.546605 \\
\hline 2 & 8 & -1.970113 & -0.356316 & -0.174753 \\
\hline 3 & 7 & -3.862402 & -1.558162 & 0.195740 \\
\hline 4 & 1 & -3.469323 & -0.566429 & -1.543637 \\
\hline 5 & 1 & -3.594491 & -1.834353 & 1.133211 \\
\hline 6 & 1 & -4.752688 & -1.887060 & -0.157424 \\
\hline 7 & 1 & 2.637788 & -2.643676 & 0.523001 \\
\hline 8 & 6 & 2.796592 & -1.582975 & 0.245083 \\
\hline 9 & 8 & 1.843146 & -0.788368 & 0.195284 \\
\hline 10 & 7 & 4.037203 & -1.332981 & -0.151021 \\
\hline 11 & 1 & 4.864810 & -1.530173 & 0.419311 \\
\hline 12 & 20 & 0.036077 & 0.541645 & 0.033649 \\
\hline 13 & 8 & 0.630596 & 1.918261 & -1.792461 \\
\hline 14 & 1 & 0.130482 & 2.651769 & -2.178880 \\
\hline 15 & 1 & 1.428900 & 1.841363 & -2.334981 \\
\hline 16 & 8 & -0.139931 & 2.072794 & 1.824498 \\
\hline 17 & 1 & 0.515986 & 2.722384 & 2.116815 \\
\hline 18 & 1 & -0.891157 & 2.183364 & 2.425150 \\
\hline
\end{tabular}

Energy: -1169.3986843a.u.

Enthalpy: -1169.251983 a.u.

Table S10: B3LYP/6-311++G** optimized structure for $\mathbf{F F}^{\text {ts }}-\mathrm{Ca}^{2+}\left(\mathrm{H}_{2} \mathrm{O}\right)_{2}$. Standard orientation:

\begin{tabular}{|c|c|c|c|c|}
\hline \multirow{2}{*}{$\begin{array}{l}\text { Center } \\
\text { Number }\end{array}$} & \multirow{2}{*}{$\begin{array}{l}\text { Atomic } \\
\text { Number }\end{array}$} & \multicolumn{3}{|c|}{ Coordinates (Angstroms) } \\
\hline & & $\mathrm{X}$ & Y & $\mathrm{Z}$ \\
\hline 1 & 6 & -1.187550 & -0.107271 & -2.234736 \\
\hline 2 & 8 & -1.215774 & -0.060126 & -0.992891 \\
\hline
\end{tabular}




$\begin{array}{rrrrr}3 & 7 & -0.114344 & -0.013590 & -3.003811 \\ 4 & 1 & -2.137673 & -0.165491 & -2.796124 \\ 5 & 1 & 1.038024 & 0.147543 & -2.525560 \\ 6 & 1 & -0.186089 & -0.274415 & -3.986952 \\ 7 & 1 & 3.480413 & -0.093992 & -0.469066 \\ 8 & 6 & 2.410459 & -0.046413 & -0.741948 \\ 9 & 8 & 1.556214 & 0.032840 & 0.157687 \\ 10 & 7 & 2.191741 & 0.000398 & -2.046415 \\ 11 & 1 & 2.936985 & -0.280524 & -2.683046 \\ 12 & 20 & -0.472687 & 0.019972 & 1.128852 \\ 13 & 8 & -0.749007 & 1.953734 & 2.443504 \\ 14 & 1 & -1.537629 & 2.239256 & 2.929031 \\ 15 & 1 & -0.095056 & 2.652959 & 2.593643 \\ 16 & 8 & -1.228609 & -1.809662 & 2.407377 \\ 17 & 1 & -0.985375 & -2.037661 & 3.317413 \\ 18 & 1 & -1.841675 & -2.506955 & 2.129900\end{array}$

Energy: -1169.362882a.u.

Enthalpy: -1169.222876a.u.

Imaginary Frequency: $-1753.465 \mathrm{~cm}^{-1}$

Table S11: B3LYP/6-311++G** optimized structure for FF-Ca ${ }^{2+}\left(\mathrm{H}_{2} \mathrm{O}\right)_{3}$. Standard orientation:

\begin{tabular}{ccccc} 
Center & Atomic & \multicolumn{3}{c}{ Coordinates (Angstroms) } \\
Number & Number & $\mathrm{X}$ & $\mathrm{Y}$ & $\mathrm{Z}$ \\
- & - & -2.026756 & 2.342536 & -0.413608 \\
\hline 1 & 6 & -1.544306 & 1.214760 & -0.255872 \\
2 & 8 & -1.641323 & 3.451032 & 0.223529 \\
3 & 7 & -2.763905 & 2.539204 & -1.217200 \\
4 & 1 & 3.175371 & 2.126023 & -0.231082 \\
5 & 1 & -2.321335 & 4.056338 & 0.694273 \\
6 & 1 & 3.789574 & -0.740806 & 0.203806 \\
7 & 1 & 3.113944 & 0.108468 & 0.054028 \\
8 & 6 & 1.875357 & -0.060421 & 0.025137 \\
9 & 8 & 3.709473 & 1.277464 & -0.087612 \\
10 & 7 & 4.718267 & 1.351946 & -0.055897 \\
11 & 1 & -2.299525 & -2.038973 & 0.077733 \\
12 & 8 & -3.202088 & -1.707835 & -0.026355 \\
13 & 1 & -2.388645 & -2.995073 & 0.193076 \\
14 & 1 & -0.122788 & -1.329764 & 2.305987 \\
15 & 8 & 0.656736 & -1.255971 & 2.872849 \\
16 & 1 & -0.822775 & -1.685307 & 2.869935 \\
17 & 1 & & &
\end{tabular}




$\begin{array}{rrrrr}18 & 8 & 0.054552 & -1.767714 & -2.069034 \\ 19 & 1 & -0.601238 & -2.237443 & -2.601655 \\ 20 & 1 & 0.874263 & -1.794479 & -2.580556 \\ 21 & 20 & -0.291037 & -0.680259 & 0.020663\end{array}$

Energy: -1245.8964917a.u.

Enthalpy: -1245.722122a.u.

Table S12: B3LYP/6-311++G** optimized structure for $\mathbf{F F}^{\mathrm{ts}}-\mathrm{Ca}^{2+}\left(\mathrm{H}_{2} \mathrm{O}\right)_{3}$. Standard orientation:

\begin{tabular}{|c|c|c|c|c|}
\hline \multirow{2}{*}{$\begin{array}{l}\text { Center } \\
\text { Number }\end{array}$} & \multirow{2}{*}{$\begin{array}{l}\text { Atomic } \\
\text { Number }\end{array}$} & \multicolumn{3}{|c|}{ Coordinates (Angstroms) } \\
\hline & & $\mathrm{X}$ & Y & $\mathrm{Z}$ \\
\hline 1 & 6 & -0.820692 & 0.117125 & -2.523102 \\
\hline 2 & 8 & -0.928050 & 0.127257 & -1.277601 \\
\hline 3 & 7 & 0.247668 & -0.021991 & -3.252971 \\
\hline 4 & 1 & -1.755388 & 0.242878 & -3.107620 \\
\hline 5 & 1 & 1.375737 & -0.173153 & -2.708978 \\
\hline 6 & 1 & 0.168702 & -0.008270 & -4.266899 \\
\hline 7 & 1 & 3.514207 & -0.464139 & -0.368541 \\
\hline 8 & 6 & 2.501370 & -0.328573 & -0.800322 \\
\hline 9 & 8 & 1.553958 & -0.205720 & 0.006756 \\
\hline 10 & 7 & 2.473467 & -0.320589 & -2.100762 \\
\hline 11 & 1 & 3.338607 & -0.433545 & -2.623512 \\
\hline 12 & 8 & 0.368410 & -0.059123 & 3.204218 \\
\hline 13 & 1 & -0.091199 & -0.001301 & 4.053770 \\
\hline 14 & 1 & 1.301765 & -0.183124 & 3.428504 \\
\hline 15 & 8 & -1.431293 & 2.202367 & 1.389045 \\
\hline 16 & 1 & -2.043723 & 2.682406 & 0.813910 \\
\hline 17 & 1 & -1.261863 & 2.795829 & 2.134153 \\
\hline 18 & 8 & -1.955446 & -1.763592 & 1.376188 \\
\hline 19 & 1 & -1.946014 & -2.385985 & 2.116883 \\
\hline 20 & 1 & -2.671698 & -2.063815 & 0.799008 \\
\hline 21 & 20 & -0.496088 & 0.062473 & 0.977858 \\
\hline
\end{tabular}

Energy: -1245.86269932a.u.

Enthalpy: -1245.700186a.u.

Imaginary Frequency: $-1518.9625 \mathrm{~cm}^{-1}$

Table S13: B3LYP/6-311++G** optimized structure for FF-Ca ${ }^{2+}\left(\mathrm{H}_{2} \mathrm{O}\right)_{4}$. Standard orientation:

Center Atomic Coordinates (Angstroms)




\begin{tabular}{|c|c|c|c|c|}
\hline Number & Number & $\mathrm{X}$ & $\mathrm{Y}$ & Z \\
\hline 1 & 6 & 1.807077 & -2.297708 & -0.262777 \\
\hline 2 & 8 & 0.695348 & -1.781900 & -0.216502 \\
\hline 3 & 7 & 2.912674 & -1.780459 & 0.338783 \\
\hline 4 & 1 & 2.007651 & -3.184286 & -0.893820 \\
\hline 5 & 1 & 3.334113 & 0.665068 & 0.016453 \\
\hline 6 & 1 & 3.459510 & -2.433480 & 0.913713 \\
\hline 7 & 1 & 1.887235 & 3.246381 & -0.189733 \\
\hline 8 & 6 & 1.946205 & 2.155446 & -0.106017 \\
\hline 9 & 8 & 0.910888 & 1.464083 & -0.058714 \\
\hline 10 & 7 & 3.174538 & 1.664000 & -0.062464 \\
\hline 11 & 1 & 3.977009 & 2.277976 & -0.105460 \\
\hline 12 & 8 & -1.244496 & -0.151618 & -2.373505 \\
\hline 13 & 1 & -0.908636 & -0.820789 & -2.983954 \\
\hline 14 & 1 & -1.659021 & 0.521637 & -2.929189 \\
\hline 15 & 8 & -1.021022 & -0.137471 & 2.425047 \\
\hline 16 & 1 & -1.341002 & 0.575876 & 2.993317 \\
\hline 17 & 1 & -0.699241 & -0.820937 & 3.027002 \\
\hline 18 & 1 & -3.291182 & -1.741393 & -0.638375 \\
\hline 19 & 8 & -2.832404 & -1.343289 & 0.112863 \\
\hline 20 & 1 & -3.271959 & -1.685618 & 0.901908 \\
\hline 21 & 8 & -2.315158 & 1.928319 & 0.086476 \\
\hline 22 & 1 & -2.043714 & 2.855352 & 0.064146 \\
\hline 23 & 1 & -3.280495 & 1.935180 & 0.129606 \\
\hline 24 & 20 & -0.842285 & 0.022642 & 0.008379 \\
\hline
\end{tabular}

Energy: -1322.3888125a.u.

Enthalpy: -1322.186725 a.u.

Table S14: B3LYP/6-311++G** optimized structure for $\mathbf{F F}^{\text {ts }}-\mathrm{Ca}^{2+}\left(\mathrm{H}_{2} \mathrm{O}\right)_{4}$. Standard orientation:

\begin{tabular}{ccccc} 
Center & Atomic & \multicolumn{3}{c}{ Coordinates (Angstroms) } \\
Number & Number & X & Y & Z \\
\hline--1 & 6 & -0.820692 & 0.117125 & -2.523102 \\
1 & 8 & -0.928050 & 0.127257 & -1.277601 \\
3 & 7 & 0.247668 & -0.021991 & -3.252971 \\
4 & 1 & -1.755388 & 0.242878 & -3.107620 \\
5 & 1 & 1.375737 & -0.173153 & -2.708978 \\
6 & 1 & 0.168702 & -0.008270 & -4.266899 \\
7 & 1 & 3.514207 & -0.464139 & -0.368541 \\
8 & 6 & 2.501370 & -0.328573 & -0.800322
\end{tabular}




\begin{tabular}{rrrrr}
9 & 8 & 1.553958 & -0.205720 & 0.006756 \\
10 & 7 & 2.473467 & -0.320589 & -2.100762 \\
11 & 1 & 3.338607 & -0.433545 & -2.623512 \\
12 & 8 & 0.368410 & -0.059123 & 3.204218 \\
13 & 1 & -0.091199 & -0.001301 & 4.053770 \\
14 & 1 & 1.301765 & -0.183124 & 3.428504 \\
15 & 8 & -1.431293 & 2.202367 & 1.389045 \\
16 & 1 & -2.043723 & 2.682406 & 0.813910 \\
17 & 1 & -1.261863 & 2.795829 & 2.134153 \\
18 & 8 & -1.955446 & -1.763592 & 1.376188 \\
19 & 1 & -1.946014 & -2.385985 & 2.116883 \\
20 & 1 & -2.671698 & -2.063815 & 0.799008 \\
21 & 20 & -0.496088 & 0.062473 & 0.977858 \\
\hline & & & &
\end{tabular}

Energy: -1322.3639963a.u.

Enthalpy: -1322.168396a.u.

Imaginary Frequency: $-1415.5351 \mathrm{~cm}^{-1}$

Table S15: B3LYP/6-311++ $\mathrm{G}^{* *}$ optimized structure for FF-Ca ${ }^{2+}\left(\mathrm{H}_{2} \mathrm{O}\right) 5$. Standard orientation:

\begin{tabular}{ccccc} 
Center & Atomic & \multicolumn{3}{c}{ Coordinates (Angstroms) } \\
Number & Number & X & Y \\
- & & -1.997683 & 2.191391 & 0.166713 \\
1 & 6 & -0.857269 & 1.776797 & 0.324434 \\
3 & 8 & -2.964343 & 1.536897 & -0.543717 \\
4 & 7 & -2.359315 & 3.103530 & 0.679337 \\
5 & 1 & -3.380828 & -0.698815 & -0.105976 \\
6 & 1 & -3.457988 & 2.115010 & -1.235367 \\
7 & 1 & -1.968675 & -3.219427 & 0.563759 \\
8 & 1 & -2.013205 & -2.164975 & 0.265946 \\
9 & 6 & -0.970457 & -1.521324 & 0.049846 \\
10 & 8 & -3.237051 & -1.667736 & 0.161897 \\
11 & 7 & -4.045340 & -2.248184 & 0.340078 \\
12 & 1 & 2.918622 & -0.901274 & 0.877146 \\
13 & 8 & 3.512518 & -0.611308 & 1.580210 \\
14 & 1 & 3.280969 & -1.726618 & 0.531233 \\
15 & 1 & 0.811457 & -0.047830 & -0.004372 \\
16 & 20 & 0.832973 & 0.772638 & -2.301233 \\
17 & 8 & 1.198290 & 1.639718 & -2.516793 \\
18 & 1 & 0.586551 & 0.362478 & -3.138951 \\
19 & 1 & 0.557669 & 0.161278 & 2.425179 \\
20 & 1 & 0.821950 & -0.476207 & 3.099793
\end{tabular}




$\begin{array}{ccccc}21 & 1 & 0.075459 & 0.855282 & 2.890405 \\ 22 & 1 & 3.047479 & 2.211311 & -0.000004 \\ 23 & 8 & 2.092874 & 2.068906 & 0.026250 \\ 24 & 1 & 1.710571 & 2.890266 & 0.360292 \\ 25 & 8 & 1.560197 & -2.043982 & -1.336561 \\ 26 & 1 & 0.984457 & -2.818890 & -1.323988 \\ 27 & 1 & 2.162990 & -2.168481 & -2.080275\end{array}$

Energy: -1398.8702591a.u.

Enthalpy: -1398.640549a.u.

Table S16: B3LYP/6-311++G** optimized structure for $\mathbf{F F}^{\mathrm{ts}}-\mathrm{Ca}^{2+}\left(\mathrm{H}_{2} \mathrm{O}\right)_{5}$. Standard orientation:

\begin{tabular}{|c|c|c|c|c|}
\hline \multirow{2}{*}{$\begin{array}{l}\text { Center } \\
\text { Number }\end{array}$} & \multirow{2}{*}{$\begin{array}{l}\text { Atomic } \\
\text { Number }\end{array}$} & \multicolumn{3}{|c|}{ Coordinates (Angstroms) } \\
\hline & & $\mathrm{X}$ & Y & $\mathrm{Z}$ \\
\hline 1 & 6 & -0.491939 & 0.067798 & -2.809518 \\
\hline 2 & 8 & -0.519305 & 0.026132 & -1.540947 \\
\hline 3 & 7 & 0.546754 & 0.103475 & -3.575444 \\
\hline 4 & 1 & -1.489022 & 0.073327 & -3.284382 \\
\hline 5 & 1 & 1.703830 & 0.081796 & -3.044039 \\
\hline 6 & 1 & 0.377533 & 0.131855 & -4.576392 \\
\hline 7 & 1 & 3.642465 & 0.047694 & -0.489473 \\
\hline 8 & 6 & 2.698619 & 0.066559 & -1.062808 \\
\hline 9 & 8 & 1.652403 & 0.102731 & -0.344518 \\
\hline 10 & 7 & 2.774167 & 0.048394 & -2.351632 \\
\hline 11 & 1 & 3.706459 & 0.023209 & -2.753708 \\
\hline 12 & 8 & -1.141958 & -0.846621 & 3.049575 \\
\hline 13 & 1 & -2.091643 & -0.954580 & 3.190643 \\
\hline 14 & 1 & -0.715327 & -1.104709 & 3.876290 \\
\hline 15 & 20 & -0.417667 & -0.025949 & 0.834826 \\
\hline 16 & 8 & -1.234933 & 2.263600 & 0.716084 \\
\hline 17 & 1 & -1.634380 & 2.723620 & -0.032920 \\
\hline 18 & 1 & -0.985116 & 2.953319 & 1.344348 \\
\hline 19 & 8 & -0.219373 & -2.425623 & 0.614566 \\
\hline 20 & 1 & -0.379637 & -3.064085 & 1.320766 \\
\hline 21 & 1 & 0.137439 & -2.930845 & -0.126720 \\
\hline 22 & 1 & -3.532223 & 0.311564 & 0.826506 \\
\hline 23 & 8 & -2.868419 & -0.388139 & 0.773048 \\
\hline 24 & 1 & -3.324770 & -1.156450 & 0.405954 \\
\hline 25 & 8 & 1.034242 & 1.116558 & 2.449791 \\
\hline 26 & 1 & 1.910833 & 1.458210 & 2.232937 \\
\hline 27 & 1 & 0.919113 & 1.266723 & 3.396371 \\
\hline
\end{tabular}


Energy: -1398.84850093a.u.

Enthalpy: -1398.624070a.u.

Imaginary Frequency: $-1323.7916 \mathrm{~cm}^{-1}$

Table S17: B3LYP/6-311++G** optimized structure for FF- $\mathrm{Mg}^{2+}\left(\mathrm{H}_{2} \mathrm{O}\right)_{1}$. Standard orientation:

\begin{tabular}{|c|c|c|c|c|}
\hline \multirow{2}{*}{$\begin{array}{l}\text { Center } \\
\text { Number }\end{array}$} & \multirow{2}{*}{$\begin{array}{l}\text { Atomic } \\
\text { Number }\end{array}$} & \multicolumn{3}{|c|}{ Coordinates (Angstroms) } \\
\hline & & $\mathrm{X}$ & $\mathrm{Y}$ & $\mathrm{Z}$ \\
\hline 1 & 6 & -2.842809 & -0.908156 & 0.394639 \\
\hline 2 & 8 & -1.742056 & -0.338541 & 0.215080 \\
\hline 3 & 7 & -3.859645 & -0.894167 & -0.425800 \\
\hline 4 & 1 & -3.074408 & -1.384477 & 1.367239 \\
\hline 5 & 1 & 3.797204 & -0.618166 & 1.279585 \\
\hline 6 & 1 & -4.465680 & -1.662394 & -0.726015 \\
\hline 7 & 1 & 2.880155 & -1.070647 & -1.505895 \\
\hline 8 & 6 & 2.777525 & -0.719699 & -0.476421 \\
\hline 9 & 8 & 1.707914 & -0.159916 & -0.098884 \\
\hline 10 & 7 & 3.805524 & -0.910254 & 0.307459 \\
\hline 11 & 1 & 4.643674 & -1.362620 & -0.041405 \\
\hline 12 & 8 & -0.204039 & 2.513465 & -0.013938 \\
\hline 13 & 1 & -1.022246 & 3.033802 & 0.019597 \\
\hline 14 & 1 & 0.525952 & 3.150078 & -0.069705 \\
\hline 15 & 12 & -0.050721 & 0.516036 & 0.014802 \\
\hline
\end{tabular}

Energy: -615.3297842a.u.

Enthalpy: -615.209896a.u.

Table S18: B3LYP/6-311++G** optimized structure for $\mathbf{F F}^{\text {ts }}-\mathrm{Mg}^{2+}\left(\mathrm{H}_{2} \mathrm{O}\right)_{1}$. Standard orientation:

\begin{tabular}{|c|c|c|c|c|}
\hline \multirow{2}{*}{$\begin{array}{l}\text { Center } \\
\text { Number }\end{array}$} & \multirow{2}{*}{$\begin{array}{l}\text { Atomic } \\
\text { Number }\end{array}$} & \multicolumn{3}{|c|}{ Coordinates (Angstroms) } \\
\hline & & $\mathrm{X}$ & Y & $\mathrm{Z}$ \\
\hline 1 & 6 & -1.451603 & -0.180548 & -1.653841 \\
\hline 2 & 8 & -1.443037 & -0.177396 & -0.403477 \\
\hline 3 & 7 & -0.392086 & -0.169527 & -2.441239 \\
\hline 4 & 1 & -2.417959 & -0.269175 & -2.179056 \\
\hline 5 & 1 & 0.785614 & -0.185481 & -1.992764 \\
\hline 6 & 1 & -0.503436 & 0.050394 & -3.432712 \\
\hline 7 & 1 & 3.233162 & 0.462125 & -0.019261 \\
\hline 8 & 6 & 2.172442 & 0.288436 & -0.268772 \\
\hline
\end{tabular}




$\begin{array}{rrrrr}9 & 8 & 1.343642 & 0.183239 & 0.661561 \\ 10 & 7 & 1.924771 & 0.130287 & -1.555761 \\ 11 & 1 & 2.607740 & 0.452988 & -2.243658 \\ 12 & 8 & -1.181055 & -0.068828 & 3.113555 \\ 13 & 1 & -2.108676 & -0.180481 & 3.382175 \\ 14 & 1 & -0.664922 & 0.006360 & 3.933957 \\ 15 & 12 & -0.478479 & -0.017125 & 1.257741\end{array}$

Energy: -615.2850818a.u.

Enthalpy: -615.1718840a.u.

Imaginary Frequency: $-1806.643 \mathrm{~cm}^{-1}$

Table S19: B3LYP/6-311++ $\mathrm{G}^{* *}$ optimized structure for FF- $\mathrm{Mg}^{2+}\left(\mathrm{H}_{2} \mathrm{O}\right)_{2}$. Standard orientation:

\begin{tabular}{|c|c|c|c|c|}
\hline \multirow{2}{*}{$\begin{array}{l}\text { Center } \\
\text { Number }\end{array}$} & \multirow{2}{*}{$\begin{array}{l}\text { Atomic } \\
\text { Number }\end{array}$} & \multicolumn{3}{|c|}{ Coordinates (Angstroms) } \\
\hline & & $\mathrm{X}$ & $\mathrm{Y}$ & Z \\
\hline 1 & 6 & 2.648990 & -1.135822 & -0.329494 \\
\hline 2 & 8 & 1.741429 & -0.285768 & -0.302082 \\
\hline 3 & 7 & 2.704886 & -2.240483 & 0.393921 \\
\hline 4 & 1 & 3.448994 & -1.073210 & -1.092339 \\
\hline 5 & 1 & -2.682278 & -2.244990 & -0.951233 \\
\hline 6 & 1 & 3.525358 & -2.548965 & 0.924139 \\
\hline 7 & 1 & -3.408006 & -0.116523 & 0.980396 \\
\hline 8 & 6 & -2.735071 & -0.635321 & 0.292054 \\
\hline 9 & 8 & -1.567439 & -0.209925 & 0.101140 \\
\hline 10 & 7 & -3.229828 & -1.698410 & -0.295697 \\
\hline 11 & 1 & -4.178689 & -1.999249 & -0.106214 \\
\hline 12 & 8 & 0.306542 & 1.920895 & 1.657290 \\
\hline 13 & 1 & 0.916774 & 2.665498 & 1.762825 \\
\hline 14 & 1 & -0.169869 & 1.835623 & 2.495746 \\
\hline 15 & 8 & 0.166292 & 2.073445 & -1.529170 \\
\hline 16 & 1 & -0.486481 & 2.750522 & -1.759328 \\
\hline 17 & 1 & 0.840456 & 2.091098 & -2.223921 \\
\hline 18 & 12 & 0.100852 & 0.737511 & 0.007465 \\
\hline
\end{tabular}

Energy: -691.8475002a.u.

Enthalpy: -691.699812a.u.

Table S20: B3LYP/6-311++G** optimized structure for $\mathbf{F F}^{\mathrm{ts}}-\mathrm{Mg}^{2+}\left(\mathrm{H}_{2} \mathrm{O}\right)_{2}$. Standard orientation:

Center Atomic Coordinates (Angstroms)




\begin{tabular}{|c|c|c|c|c|}
\hline Number & Number & $\mathrm{X}$ & Y & Z \\
\hline 1 & 6 & -1.310326 & -0.231618 & -1.907267 \\
\hline 2 & 8 & -1.331094 & -0.201895 & -0.662384 \\
\hline 3 & 7 & -0.236043 & -0.285407 & -2.677333 \\
\hline 4 & 1 & -2.266289 & -0.291433 & -2.456314 \\
\hline 5 & 1 & 0.927361 & -0.330730 & -2.202271 \\
\hline 6 & 1 & -0.326949 & -0.086026 & -3.674079 \\
\hline 7 & 1 & 3.334638 & 0.370459 & -0.203756 \\
\hline 8 & 6 & 2.275106 & 0.212910 & -0.469954 \\
\hline 9 & 8 & 1.418681 & 0.212372 & 0.434052 \\
\hline 10 & 7 & 2.065745 & -0.041469 & -1.751624 \\
\hline 11 & 1 & 2.785213 & 0.206127 & -2.431955 \\
\hline 12 & 8 & -0.821473 & 1.695158 & 2.183796 \\
\hline 13 & 1 & -1.655100 & 1.926352 & 2.621397 \\
\hline 14 & 1 & -0.204564 & 2.420114 & 2.366568 \\
\hline 15 & 8 & -0.981624 & -1.517863 & 2.216301 \\
\hline 16 & 1 & -0.708674 & -1.711759 & 3.126035 \\
\hline 17 & 1 & -1.551183 & -2.251039 & 1.937508 \\
\hline 18 & 12 & -0.433913 & 0.053844 & 1.067397 \\
\hline
\end{tabular}

Energy: -691.811726a.u.

Enthalpy: -691.670566a.u.

Imaginary Frequency: $-1770.5170 \mathrm{~cm}^{-1}$

Table S21: B3LYP/6-311++ $\mathrm{G}^{* *}$ optimized structure for FF- $\mathrm{Mg}^{2+}\left(\mathrm{H}_{2} \mathrm{O}\right)_{3}$. Standard orientation:

\begin{tabular}{ccccc} 
Center & Atomic & \multicolumn{3}{c}{ Coordinates (Angstroms) } \\
Number & Number & X & Y & Z \\
------------------- \\
\hline 1 & 6 & -0.703980 & 2.450416 & -0.323508 \\
2 & 8 & 0.229689 & 1.645305 & -0.304269 \\
3 & 7 & -1.899112 & 2.249839 & 0.272245 \\
4 & 1 & -0.645614 & 3.368939 & -0.936006 \\
5 & 1 & -3.058542 & 0.045725 & -0.110581 \\
6 & 1 & -2.255936 & 2.994661 & 0.884063 \\
7 & 1 & -2.142358 & -2.773301 & -0.008051 \\
8 & 6 & -1.995594 & -1.688655 & -0.028293 \\
9 & 8 & -0.840506 & -1.209464 & 0.008569 \\
10 & 7 & -3.097141 & -0.968137 & -0.091647 \\
11 & 1 & -4.004981 & -1.415433 & -0.121664 \\
12 & 8 & 2.805586 & 0.650005 & 0.041622 \\
13 & 1 & 2.915241 & 1.592417 & -0.146538
\end{tabular}




$\begin{array}{rrrrr}14 & 1 & 3.690683 & 0.287919 & 0.183759 \\ 15 & 8 & 1.497519 & -1.292235 & -1.660424 \\ 16 & 1 & 1.010201 & -2.028399 & -2.053915 \\ 17 & 1 & 2.265236 & -1.126084 & -2.223542 \\ 18 & 8 & 1.280180 & -0.927520 & 1.936148 \\ 19 & 1 & 1.964216 & -0.637288 & 2.554241 \\ 20 & 1 & 0.763822 & -1.610551 & 2.385072 \\ 21 & 12 & 0.907458 & -0.264150 & 0.022218\end{array}$

Energy: -768.3449844a.u.

Enthalpy: -768.169226a.u.

Table S22: B3LYP/6-311++G** optimized structure for $\mathbf{F F}^{\text {ts }}-\mathrm{Mg}^{2+}\left(\mathrm{H}_{2} \mathrm{O}\right)_{3}$. Standard orientation:

\begin{tabular}{|c|c|c|c|c|}
\hline \multirow{2}{*}{$\begin{array}{l}\text { Center } \\
\text { Number }\end{array}$} & \multirow{2}{*}{$\begin{array}{l}\text { Atomic } \\
\text { Number }\end{array}$} & \multicolumn{3}{|c|}{ Coordinates (Angstroms) } \\
\hline & & $\mathrm{X}$ & $\mathrm{Y}$ & $\mathrm{Z}$ \\
\hline 1 & 6 & -0.958256 & -0.049483 & -2.250872 \\
\hline 2 & 8 & -1.122748 & -0.021325 & -1.019694 \\
\hline 3 & 7 & 0.180502 & 0.095921 & -2.909177 \\
\hline 4 & 1 & -1.842511 & -0.134423 & -2.907347 \\
\hline 5 & 1 & 1.272614 & 0.282296 & -2.316986 \\
\hline 6 & 1 & 0.217752 & -0.161523 & -3.895168 \\
\hline 7 & 1 & 3.462055 & -0.087990 & -0.022672 \\
\hline 8 & 6 & 2.429627 & -0.021049 & -0.410366 \\
\hline 9 & 8 & 1.482904 & -0.006864 & 0.391925 \\
\hline 10 & 7 & 2.363797 & 0.122794 & -1.726238 \\
\hline 11 & 1 & 3.174711 & -0.128089 & -2.291390 \\
\hline 12 & 8 & -2.514589 & -0.004034 & 1.421437 \\
\hline 13 & 1 & -3.203141 & 0.007957 & 0.741792 \\
\hline 14 & 1 & -2.963774 & 0.002081 & 2.277694 \\
\hline 15 & 8 & -0.186522 & 1.729939 & 1.935441 \\
\hline 16 & 1 & 0.681524 & 2.088672 & 2.166153 \\
\hline 17 & 1 & -0.846371 & 2.357362 & 2.260748 \\
\hline 18 & 8 & -0.209750 & -1.773770 & 1.923082 \\
\hline 19 & 1 & -0.883398 & -2.391989 & 2.237737 \\
\hline 20 & 1 & 0.648591 & -2.138578 & 2.178883 \\
\hline 21 & 12 & -0.495894 & -0.016263 & 0.897363 \\
\hline
\end{tabular}

Energy: - 768.3184986a.u.

Enthalpy: -768.149415 a.u.

Imaginary Frequency: $-1713.5591 \mathrm{~cm}^{-1}$ 
Table S23: B3LYP/6-311++G** optimized structure for FF- $\mathrm{Mg}^{2+}\left(\mathrm{H}_{2} \mathrm{O}\right)_{4}$. Standard orientation:

\begin{tabular}{|c|c|c|c|c|}
\hline \multirow{2}{*}{$\begin{array}{l}\text { Center } \\
\text { Number }\end{array}$} & \multirow{2}{*}{$\begin{array}{l}\text { Atomic } \\
\text { Number }\end{array}$} & \multicolumn{3}{|c|}{ Coordinates (Angstroms) } \\
\hline & & $X$ & Y & 2 \\
\hline 1 & 6 & 1.874856 & -1.898957 & 0.022131 \\
\hline 2 & 8 & 0.862515 & -1.171369 & 0.042876 \\
\hline 3 & 7 & 3.107757 & -1.440586 & -0.098270 \\
\hline 4 & 1 & 1.784698 & -2.987785 & 0.105562 \\
\hline 5 & 1 & 3.279140 & -0.443566 & -0.177120 \\
\hline 6 & 1 & 3.897880 & -2.072485 & -0.112094 \\
\hline 7 & 1 & 1.308890 & 3.341106 & -0.771953 \\
\hline 8 & 6 & 1.210517 & 2.361136 & -0.267560 \\
\hline 9 & 8 & 0.145411 & 1.751153 & -0.278589 \\
\hline 10 & 7 & 2.378103 & 1.883090 & 0.236022 \\
\hline 11 & 1 & 2.870743 & 2.512525 & 0.882190 \\
\hline 12 & 8 & -1.029440 & -0.395765 & -2.078260 \\
\hline 13 & 1 & -0.405800 & -0.869900 & -2.642621 \\
\hline 14 & 1 & -1.603106 & 0.114028 & -2.664888 \\
\hline 15 & 8 & -0.860161 & 0.013257 & 2.130686 \\
\hline 16 & 1 & -1.430234 & 0.584098 & 2.661779 \\
\hline 17 & 1 & -0.235960 & -0.404573 & 2.737199 \\
\hline 18 & 1 & -2.531200 & -2.269051 & -0.517387 \\
\hline 19 & 8 & -2.234500 & -1.708461 & 0.211028 \\
\hline 20 & 1 & -2.473666 & -2.161603 & 1.029498 \\
\hline 21 & 8 & -2.540824 & 1.153240 & -0.035765 \\
\hline 22 & 1 & -2.514034 & 2.108020 & -0.180053 \\
\hline 23 & 1 & -3.468564 & 0.894181 & 0.033985 \\
\hline 24 & 12 & -0.844671 & -0.112670 & 0.015699 \\
\hline
\end{tabular}

Energy: -844.8394735a.u.

Enthalpy: -844.636066 a.u.

Table S24: B3LYP/6-311++G** optimized structure for $\mathbf{F F}^{\text {ts }}-\mathrm{Mg}^{2+}\left(\mathrm{H}_{2} \mathrm{O}\right)_{4}$. Standard orientation:

\begin{tabular}{|c|c|c|c|c|}
\hline \multirow{2}{*}{$\begin{array}{l}\text { Center } \\
\text { Number }\end{array}$} & \multirow{2}{*}{$\begin{array}{l}\text { Atomic } \\
\text { Number }\end{array}$} & \multicolumn{3}{|c|}{ Coordinates (Angstroms) } \\
\hline & & $\mathrm{X}$ & $\mathrm{Y}$ & Z \\
\hline 1 & 6 & -0.929457 & -0.000008 & -2.362108 \\
\hline 2 & 8 & -0.966399 & -0.000004 & -1.111747 \\
\hline 3 & 7 & 0.106275 & -0.000012 & -3.148088 \\
\hline 4 & 1 & -1.907545 & -0.000010 & -2.884580 \\
\hline
\end{tabular}




\begin{tabular}{rrrrc}
5 & 1 & 1.266524 & -0.000010 & -2.641637 \\
6 & 1 & -0.030692 & -0.000015 & -4.155846 \\
7 & 1 & 3.452573 & -0.000002 & -0.328836 \\
8 & 6 & 2.423434 & -0.000004 & -0.743996 \\
9 & 8 & 1.492110 & 0.000000 & 0.086277 \\
10 & 7 & 2.375563 & -0.000008 & -2.044887 \\
11 & 1 & 3.244264 & -0.000011 & -2.573576 \\
12 & 8 & -0.582740 & 2.115043 & 0.942653 \\
13 & 1 & -0.827458 & 2.694713 & 0.209877 \\
14 & 1 & -0.209176 & 2.682120 & 1.629892 \\
15 & 8 & -0.582741 & -2.115036 & 0.942669 \\
16 & 1 & -0.209178 & -2.682108 & 1.629912 \\
17 & 1 & -0.827460 & -2.694711 & 0.209897 \\
18 & 1 & -2.910181 & 0.777152 & 1.845096 \\
19 & 8 & -2.348618 & 0.000007 & 1.727345 \\
20 & 1 & -2.910182 & -0.777136 & 1.845102 \\
21 & 8 & 0.413760 & 0.000010 & 2.798899 \\
22 & 1 & 1.356099 & 0.000011 & 3.012322 \\
23 & 1 & -0.068194 & 0.000014 & 3.636602 \\
24 & 12 & -0.429925 & 0.000003 & 0.872038 \\
\hline-----------------------------------------------------------
\end{tabular}

Energy: -844.617615a.u.

Enthalpy: -844.813501a.u.

Imaginary Frequency: $-1482.4553 \mathrm{~cm}^{-1}$

Table S25: B3LYP/6-311++G** optimized structure for FF-Na ${ }^{+}$. Standard orientation:

\begin{tabular}{|c|c|c|c|c|}
\hline \multirow{2}{*}{$\begin{array}{l}\text { Center } \\
\text { Number }\end{array}$} & \multirow{2}{*}{$\begin{array}{l}\text { Atomic } \\
\text { Number }\end{array}$} & \multicolumn{3}{|c|}{ Coordinates (Angstroms) } \\
\hline & & $\mathrm{X}$ & $\mathrm{Y}$ & $\mathrm{Z}$ \\
\hline 1 & 6 & -2.343282 & -0.086266 & -0.039110 \\
\hline 2 & 8 & -1.491143 & 0.801713 & 0.034881 \\
\hline 3 & 7 & -2.064637 & -1.390351 & -0.107332 \\
\hline 4 & 1 & -3.414900 & 0.152553 & -0.053790 \\
\hline 5 & 1 & -1.101818 & -1.706800 & -0.098815 \\
\hline 6 & 1 & -2.802861 & -2.075437 & -0.169646 \\
\hline 7 & 1 & 3.162453 & -1.002136 & -0.742764 \\
\hline 8 & 6 & 2.258802 & -0.651291 & -0.208468 \\
\hline 9 & 8 & 1.964792 & 0.529628 & -0.149017 \\
\hline 10 & 7 & 1.464323 & -1.659656 & 0.281295 \\
\hline 11 & 1 & 1.957571 & -2.293635 & 0.921934 \\
\hline 12 & 11 & 0.283586 & 2.004557 & 0.120355 \\
\hline
\end{tabular}


Energy: -501.3952959a.u.

Enthalpy: -501.304609a.u.

Table S26: B3LYP/6-311++G** optimized structure for $\mathbf{F F}^{\mathbf{t s}}-\mathrm{Na}^{+}$. Standard orientation:

\begin{tabular}{|c|c|c|c|c|}
\hline \multirow{2}{*}{$\begin{array}{l}\text { Center } \\
\text { Number }\end{array}$} & \multirow{2}{*}{$\begin{array}{l}\text { Atomic } \\
\text { Number }\end{array}$} & \multicolumn{3}{|c|}{ Coordinates (Angstroms) } \\
\hline & & $\mathrm{X}$ & $\mathrm{Y}$ & Z \\
\hline 1 & 6 & 0.495560 & 1.820905 & -0.000001 \\
\hline 2 & 8 & -0.626552 & 1.237066 & -0.000004 \\
\hline 3 & 7 & 1.668860 & 1.273367 & 0.000003 \\
\hline 4 & 1 & 0.429429 & 2.922873 & -0.000004 \\
\hline 5 & 1 & 1.760267 & 0.000003 & 0.000003 \\
\hline 6 & 1 & 2.457811 & 1.910469 & 0.000004 \\
\hline 7 & 1 & 0.429444 & -2.922871 & -0.000003 \\
\hline 8 & 6 & 0.495569 & -1.820903 & -0.000001 \\
\hline 9 & 8 & -0.626545 & -1.237068 & -0.000001 \\
\hline 10 & 7 & 1.668867 & -1.273359 & 0.000000 \\
\hline 11 & 1 & 2.457822 & -1.910457 & 0.000001 \\
\hline 12 & 11 & -2.438261 & -0.000005 & 0.000003 \\
\hline
\end{tabular}

Energy: -501.3743459a.u.

Enthalpy: -501.288369a.u.

Imaginary Frequency: $-1326.5790 \mathrm{~cm}^{-1}$

Table S27: B3LYP/6-311++G** optimized structure for FF-Na ${ }^{+}\left(\mathrm{H}_{2} \mathrm{O}\right)_{1}$. Standard orientation:

\begin{tabular}{ccccc} 
Center & Atomic & \multicolumn{3}{c}{ Coordinates (Angstroms) } \\
Number & Number & X & Y & $Z$ \\
\hdashline 1 & 6 & -1.473040 & -2.058930 & -0.053615 \\
2 & 8 & -0.343055 & -1.572006 & -0.011767 \\
3 & 7 & -2.602866 & -1.343733 & -0.051741 \\
4 & 1 & -1.624722 & -3.146265 & -0.097028 \\
5 & 1 & -2.563549 & -0.331054 & -0.009851 \\
6 & 1 & -3.502508 & -1.798486 & -0.089271 \\
7 & 1 & -0.734261 & 3.363041 & -0.731760 \\
8 & 6 & -0.624162 & 2.393815 & -0.207540 \\
9 & 8 & 0.424255 & 1.777630 & -0.204889 \\
10 & 7 & -1.792771 & 1.918095 & 0.345525 \\
11 & 1 & -2.217835 & 2.573998 & 1.012424 \\
12 & 8 & 3.669277 & -0.509721 & 0.097984
\end{tabular}




$\begin{array}{rrrrr}13 & 1 & 4.170804 & -1.330490 & 0.166778 \\ 14 & 1 & 4.328792 & 0.192228 & 0.052186 \\ 15 & 11 & 1.408376 & -0.283640 & 0.014213\end{array}$

Energy: -577.8836585a.u.

Enthalpy: -577.765436a.u.

Table S28: B3LYP/6-311++G** optimized structure for $\mathbf{F F}^{\mathbf{t s}}-\mathrm{Na}^{+}\left(\mathrm{H}_{2} \mathrm{O}\right)_{1}$. Standard orientation:

\begin{tabular}{|c|c|c|c|c|}
\hline \multirow{2}{*}{$\begin{array}{l}\text { Center } \\
\text { Number }\end{array}$} & \multirow{2}{*}{$\begin{array}{l}\text { Atomic } \\
\text { Number }\end{array}$} & \multicolumn{3}{|c|}{ Coordinates (Angstroms) } \\
\hline & & $\mathrm{X}$ & Y & Z \\
\hline 1 & 6 & -1.047078 & 0.069535 & -1.914030 \\
\hline 2 & 8 & -1.037484 & 0.016619 & -0.650352 \\
\hline 3 & 7 & -0.025115 & 0.113683 & -2.708824 \\
\hline 4 & 1 & -2.058923 & 0.077407 & -2.354760 \\
\hline 5 & 1 & 1.148861 & 0.105158 & -2.206836 \\
\hline 6 & 1 & -0.235930 & 0.153148 & -3.699658 \\
\hline 7 & 1 & 3.111049 & 0.019077 & 0.333815 \\
\hline 8 & 6 & 2.169228 & 0.033340 & -0.241429 \\
\hline 9 & 8 & 1.128352 & -0.007994 & 0.475955 \\
\hline 10 & 7 & 2.233910 & 0.088507 & -1.534035 \\
\hline 11 & 1 & 3.166728 & 0.115036 & -1.930139 \\
\hline 12 & 8 & -1.858232 & -0.170332 & 3.569332 \\
\hline 13 & 1 & -2.804688 & -0.260317 & 3.732544 \\
\hline 14 & 1 & -1.451683 & -0.129823 & 4.443170 \\
\hline 15 & 11 & -0.811906 & -0.074419 & 1.559559 \\
\hline
\end{tabular}

Energy: -577.8648283a.u.

Enthalpy: -577.751092a.u.

Imaginary Frequency: $-1314.9834 \mathrm{~cm}^{-1}$

Table S29: B3LYP/6-311++G** optimized structure for FF-Na ${ }^{+}\left(\mathrm{H}_{2} \mathrm{O}\right)_{2}$. Standard orientation:

\begin{tabular}{ccccc}
$\begin{array}{c}\text { Center } \\
\text { Number }\end{array}$ & $\begin{array}{c}\text { Atomic } \\
\text { Number }\end{array}$ & \multicolumn{4}{c}{ Coordinates (Angstroms) } \\
- & $\mathrm{X}$ & $\mathrm{Y}$ & $\mathrm{Z}$ \\
\hline 1 & 6 & -1.675814 & -2.019096 & -0.377295 \\
2 & 8 & -0.552224 & -1.555287 & -0.195881 \\
3 & 7 & -2.809164 & -1.308252 & -0.310819 \\
4 & 1 & -1.825033 & -3.082668 & -0.613484 \\
5 & 1 & -2.774842 & -0.317702 & -0.093661
\end{tabular}




\begin{tabular}{rrrrr}
6 & 1 & -3.703384 & -1.746701 & -0.469199 \\
7 & 1 & -1.141776 & 3.439311 & -0.286210 \\
8 & 6 & -0.967007 & 2.407817 & 0.079016 \\
9 & 8 & 0.106339 & 1.853252 & -0.040634 \\
10 & 7 & -2.097485 & 1.798267 & 0.585376 \\
11 & 1 & -2.522321 & 2.329362 & 1.355421 \\
12 & 1 & 2.153006 & -1.595763 & 2.442349 \\
13 & 8 & 2.385945 & -0.858874 & 1.866682 \\
14 & 1 & 3.222112 & -0.518557 & 2.203289 \\
15 & 8 & 2.728643 & 0.242622 & -1.674021 \\
16 & 1 & 2.814715 & 1.092102 & -2.120696 \\
17 & 1 & 3.348578 & -0.348330 & -2.115428 \\
18 & 11 & 1.207526 & -0.224290 & -0.007616 \\
\hline-----------------------------------------------------------------------
\end{tabular}

Energy: -654.3648915a.u.

Enthalpy: -654.219327a.u.

Table S30: B3LYP/6-311++G** optimized structure for $\mathbf{F F}^{\mathbf{t s}}-\mathrm{Na}^{+}\left(\mathrm{H}_{2} \mathrm{O}\right)_{2}$. Standard orientation:

\begin{tabular}{|c|c|c|c|c|}
\hline \multirow{2}{*}{$\begin{array}{l}\text { Center } \\
\text { Number }\end{array}$} & \multirow{2}{*}{$\begin{array}{l}\text { Atomic } \\
\text { Number }\end{array}$} & \multicolumn{3}{|c|}{ Coordinates (Angstroms) } \\
\hline & & $\mathrm{X}$ & $\mathrm{Y}$ & Z \\
\hline 1 & 6 & -1.047078 & 0.069535 & -1.914030 \\
\hline 2 & 8 & -1.037484 & 0.016619 & -0.650352 \\
\hline 3 & 7 & -0.025115 & 0.113683 & -2.708824 \\
\hline 4 & 1 & -2.058923 & 0.077407 & -2.354760 \\
\hline 5 & 1 & 1.148861 & 0.105158 & -2.206836 \\
\hline 6 & 1 & -0.235930 & 0.153148 & -3.699658 \\
\hline 7 & 1 & 3.111049 & 0.019077 & 0.333815 \\
\hline 8 & 6 & 2.169228 & 0.033340 & -0.241429 \\
\hline 9 & 8 & 1.128352 & -0.007994 & 0.475955 \\
\hline 10 & 7 & 2.233910 & 0.088507 & -1.534035 \\
\hline 11 & 1 & 3.166728 & 0.115036 & -1.930139 \\
\hline 12 & 8 & -1.858232 & -0.170332 & 3.569332 \\
\hline 13 & 1 & -2.804688 & -0.260317 & 3.732544 \\
\hline 14 & 1 & -1.451683 & -0.129823 & 4.443170 \\
\hline 15 & 11 & -0.811906 & -0.074419 & 1.559559 \\
\hline
\end{tabular}

Energy: -654.3482379a.u.

Enthalpy: -654.206871a.u.

Imaginary Frequency: $-1307.4942 \mathrm{~cm}^{-1}$ 
Table S31: B3LYP/6-311++G** optimized structure for FF-K ${ }^{+}$. Standard orientation:

\begin{tabular}{|c|c|c|c|c|}
\hline \multirow{2}{*}{$\begin{array}{l}\text { Center } \\
\text { Number }\end{array}$} & \multirow{2}{*}{$\begin{array}{l}\text { Atomic } \\
\text { Number }\end{array}$} & \multicolumn{3}{|c|}{ Coordinates (Angstroms) } \\
\hline & & $\mathrm{X}$ & $\mathrm{Y}$ & $\mathrm{Z}$ \\
\hline 1 & 6 & 1.770202 & 1.643782 & -0.238188 \\
\hline 2 & 8 & 1.885416 & 0.435951 & -0.172152 \\
\hline 3 & 7 & 0.728697 & 2.345812 & 0.331488 \\
\hline 4 & 1 & 2.460190 & 2.271221 & -0.836314 \\
\hline 5 & 1 & -1.508572 & 1.658409 & 0.032096 \\
\hline 6 & 1 & 1.046349 & 3.112201 & 0.937680 \\
\hline 7 & 1 & -3.405972 & -0.616941 & -0.177339 \\
\hline 8 & 6 & -2.402086 & -0.179427 & -0.082090 \\
\hline 9 & 8 & -1.397493 & -0.888003 & -0.009956 \\
\hline 10 & 7 & -2.387135 & 1.158226 & -0.054827 \\
\hline 11 & 1 & -3.245380 & 1.684462 & -0.117897 \\
\hline 12 & 19 & 0.850020 & -1.989860 & 0.084404 \\
\hline
\end{tabular}

Energy: -939.04699a.u.

Enthalpy: -938.956595 a.u.

Table S32: B3LYP/6-311++G** optimized structure for $\mathbf{F F}^{\text {ts }}-\mathrm{K}^{+}$. Standard orientation:

\begin{tabular}{|c|c|c|c|c|}
\hline \multirow{2}{*}{$\begin{array}{l}\text { Center } \\
\text { Number }\end{array}$} & \multirow{2}{*}{$\begin{array}{l}\text { Atomic } \\
\text { Number }\end{array}$} & \multicolumn{3}{|c|}{ Coordinates (Angstroms) } \\
\hline & & $\mathrm{X}$ & $\mathrm{Y}$ & Z \\
\hline 1 & 6 & -1.161720 & 0.000000 & -1.662180 \\
\hline 2 & 8 & -1.166818 & 0.000000 & -0.396247 \\
\hline 3 & 7 & -0.131758 & 0.000000 & -2.447928 \\
\hline 4 & 1 & -2.168845 & 0.000000 & -2.113815 \\
\hline 5 & 1 & 1.036253 & 0.000000 & -1.933435 \\
\hline 6 & 1 & -0.333395 & 0.000000 & -3.441349 \\
\hline 7 & 1 & 2.961090 & 0.000000 & 0.635650 \\
\hline 8 & 6 & 2.027368 & 0.000000 & 0.047060 \\
\hline 9 & 8 & 0.976019 & 0.000000 & 0.752238 \\
\hline 10 & 7 & 2.111399 & 0.000000 & -1.245674 \\
\hline 11 & 1 & 3.050289 & 0.000000 & -1.627813 \\
\hline 12 & 19 & -1.161598 & 0.000000 & 2.167303 \\
\hline
\end{tabular}

Energy: -939.029188a.u.

Enthalpy: -938.943139a.u.

Imaginary Frequency: $-1305.4469 \mathrm{~cm}^{-1}$ 
Table S33: B3LYP/6-311++G** optimized structure for FF-K $\mathrm{K}^{+}\left(\mathrm{H}_{2} \mathrm{O}\right)_{1}$. Standard orientation:

\begin{tabular}{|c|c|c|c|c|}
\hline \multirow{2}{*}{$\begin{array}{l}\text { Center } \\
\text { Number }\end{array}$} & \multirow{2}{*}{$\begin{array}{l}\text { Atomic } \\
\text { Number }\end{array}$} & \multicolumn{3}{|c|}{ Coordinates (Angstroms) } \\
\hline & & $\mathrm{X}$ & $\mathrm{Y}$ & $\mathrm{Z}$ \\
\hline 1 & 6 & -1.598453 & -1.910235 & 0.003468 \\
\hline 2 & 8 & -0.544647 & -1.255727 & 0.001039 \\
\hline 3 & 7 & -2.820157 & -1.402478 & 0.002638 \\
\hline 4 & 1 & -1.550310 & -3.009553 & 0.006645 \\
\hline 5 & 1 & -2.947082 & -0.374071 & -0.000793 \\
\hline 6 & 1 & -3.621056 & -2.016652 & 0.004779 \\
\hline 7 & 1 & -1.436516 & 3.059567 & -0.003029 \\
\hline 8 & 6 & -1.570290 & 1.949442 & -0.001559 \\
\hline 9 & 8 & -0.424556 & 1.405629 & 0.006051 \\
\hline 10 & 7 & -2.755058 & 1.467242 & -0.007200 \\
\hline 11 & 1 & -3.481810 & 2.177687 & -0.012595 \\
\hline 12 & 8 & 4.438209 & 0.060422 & -0.000172 \\
\hline 13 & 1 & 5.079193 & -0.652255 & -0.106956 \\
\hline 14 & 1 & 4.971755 & 0.856497 & 0.108141 \\
\hline 15 & 19 & 1.751197 & -0.126968 & -0.001634 \\
\hline
\end{tabular}

Energy: -1015.5175378a.u.

Enthalpy: -1015.400268a.u.

Table S34: B3LYP/6-311++G** optimized structure for $\mathbf{F F}^{\text {ts }}-\mathrm{K}^{+}\left(\mathrm{H}_{2} \mathrm{O}\right)_{1}$. Standard orientation:

\begin{tabular}{ccccc} 
Center & Atomic & \multicolumn{3}{c}{ Coordinates (Angstroms) } \\
Number & Number & $\mathrm{X}$ & $\mathrm{Y}$ & $\mathrm{Z}$ \\
\hline 1 & 6 & -0.764983 & -0.066093 & -2.304037 \\
2 & 8 & -0.796209 & -0.046990 & -1.039400 \\
3 & 7 & 0.280875 & -0.044556 & -3.069766 \\
4 & 1 & -1.762241 & -0.105716 & -2.775393 \\
5 & 1 & 1.436613 & 0.000111 & -2.529862 \\
6 & 1 & 0.097818 & -0.066314 & -4.066312 \\
7 & 1 & 3.286450 & 0.105768 & 0.091408 \\
8 & 6 & 2.370702 & 0.066233 & -0.523500 \\
9 & 8 & 1.300670 & 0.047230 & 0.151272 \\
10 & 7 & 2.492424 & 0.044683 & -1.813982 \\
11 & 1 & 3.442019 & 0.066353 & -2.167374 \\
12 & 8 & -2.215833 & -0.000903 & 3.901756 \\
13 & 1 & -3.109250 & -0.322231 & 4.071380
\end{tabular}




$\begin{array}{rrrrr}14 & 1 & -1.903967 & 0.319897 & 4.756183 \\ 15 & 19 & -0.886520 & 0.000300 & 1.561179\end{array}$

Energy: -1015.5104903a.u.

Enthalpy: -1015.396923a.u.

Imaginary Frequency: $-1301.4728 \mathrm{~cm}^{-1}$

Table S35: B3LYP/6-311++G** optimized structure for FF- $\mathrm{K}^{+}\left(\mathrm{H}_{2} \mathrm{O}\right)_{2}$. Standard orientation:

\begin{tabular}{|c|c|c|c|c|}
\hline \multirow{2}{*}{$\begin{array}{l}\text { Center } \\
\text { Number }\end{array}$} & \multirow{2}{*}{$\begin{array}{l}\text { Atomic } \\
\text { Number }\end{array}$} & \multicolumn{3}{|c|}{ Coordinates (Angstroms) } \\
\hline & & $\mathrm{X}$ & $\mathrm{Y}$ & Z \\
\hline 1 & 6 & -1.807812 & -1.839673 & 0.481880 \\
\hline 2 & 8 & -0.783188 & -1.167713 & 0.285467 \\
\hline 3 & 7 & -3.051397 & -1.401884 & 0.378322 \\
\hline 4 & 1 & -1.705623 & -2.897146 & 0.769409 \\
\hline 5 & 1 & -3.225862 & -0.414099 & 0.105699 \\
\hline 6 & 1 & -3.820658 & -2.029632 & 0.558664 \\
\hline 7 & 1 & -1.790031 & 2.904789 & -0.772772 \\
\hline 8 & 6 & -1.922780 & 1.835233 & -0.487071 \\
\hline 9 & 8 & -0.769362 & 1.318990 & -0.318227 \\
\hline 10 & 7 & -3.095955 & 1.336788 & -0.382790 \\
\hline 11 & 1 & -3.829157 & 2.011391 & -0.582554 \\
\hline 12 & 8 & 3.059937 & 0.671278 & 2.133331 \\
\hline 13 & 1 & 3.962436 & 0.406961 & 2.345065 \\
\hline 14 & 1 & 2.781039 & 1.219141 & 2.875951 \\
\hline 15 & 8 & 3.107940 & -0.633590 & -2.121246 \\
\hline 16 & 1 & 3.964262 & -0.263064 & -2.363371 \\
\hline 17 & 1 & 2.894574 & -1.249843 & -2.831280 \\
\hline 18 & 19 & 1.540075 & -0.037784 & 0.006474 \\
\hline
\end{tabular}

Energy: -1091.9956245a.u.

Enthalpy: -1091.850524a.u.

Table S36: B3LYP/6-311++G** optimized structure for $\mathbf{F F}^{\mathrm{ts}}-\mathrm{K}^{+}\left(\mathrm{H}_{2} \mathrm{O}\right)_{2}$. Standard orientation:

\begin{tabular}{ccccc}
$\begin{array}{c}\text { Center } \\
\text { Number }\end{array}$ & Atomic & \multicolumn{3}{c}{ Coordinates (Angstroms) } \\
- & Number & X & Y & $Z$ \\
\hline 1 & 6 & -0.614718 & 0.126531 & -2.536827 \\
2 & 8 & -0.645857 & 0.072048 & -1.274043 \\
3 & 7 & 0.429430 & 0.084137 & -3.305819
\end{tabular}




$\begin{array}{rrrrr}4 & 1 & -1.610137 & 0.221010 & -3.004267 \\ 5 & 1 & 1.581320 & -0.032424 & -2.767644 \\ 6 & 1 & 0.244579 & 0.142017 & -4.300371 \\ 7 & 1 & 3.403455 & -0.257527 & -0.134245 \\ 8 & 6 & 2.496218 & -0.164885 & -0.756034 \\ 9 & 8 & 1.424865 & -0.087637 & -0.089100 \\ 10 & 7 & 2.628725 & -0.146817 & -2.046580 \\ 11 & 1 & 3.578695 & -0.220488 & -2.391231 \\ 12 & 8 & -1.245334 & 2.242507 & 2.830487 \\ 13 & 1 & -1.975704 & 2.427390 & 3.431841 \\ 14 & 1 & -0.719063 & 3.050239 & 2.823739 \\ 15 & 8 & -1.826684 & -2.181006 & 2.545349 \\ 16 & 1 & -1.981614 & -2.347150 & 3.482173 \\ 17 & 1 & -2.089864 & -2.995686 & 2.102099 \\ 18 & 19 & -0.778088 & 0.016538 & 1.362122 \\ & 1 & -----------------------\end{array}$

Energy: -1091.9887723a.u.

Enthalpy: -1091.847762a.u.

Imaginary Frequency: $-1300.8159 \mathrm{~cm}^{-1}$

Table S37: B3LYP/6-311++G** optimized structure for FF- $\left(\mathrm{H}_{2} \mathrm{O}\right)_{1}$. Standard orientation:

\begin{tabular}{|c|c|c|c|c|}
\hline \multirow{2}{*}{$\begin{array}{l}\text { Center } \\
\text { Number }\end{array}$} & \multirow{2}{*}{$\begin{array}{l}\text { Atomic } \\
\text { Number }\end{array}$} & \multicolumn{3}{|c|}{ Coordinates (Angstroms) } \\
\hline & & $X$ & $\mathrm{Y}$ & Z \\
\hline 1 & 6 & -0.995166 & -1.757547 & 0.000553 \\
\hline 2 & 8 & 0.228606 & -1.396732 & -0.002809 \\
\hline 3 & 7 & -2.074772 & -1.059804 & 0.012179 \\
\hline 4 & 1 & -1.875619 & 0.739207 & -0.038185 \\
\hline 5 & 1 & -2.896858 & -1.655971 & 0.009398 \\
\hline 6 & 1 & -1.027205 & -2.868620 & -0.008416 \\
\hline 7 & 7 & -1.484119 & 1.703480 & -0.059591 \\
\hline 8 & 6 & -0.164747 & 1.860215 & 0.016615 \\
\hline 9 & 1 & -2.081268 & 2.513289 & -0.109239 \\
\hline 10 & 8 & 0.664685 & 0.951900 & 0.094123 \\
\hline 11 & 1 & 0.184467 & 2.905845 & 0.006279 \\
\hline 12 & 1 & 2.568209 & 0.270813 & 0.004309 \\
\hline 13 & 8 & 3.357310 & -0.286288 & -0.039657 \\
\hline 14 & 1 & 2.995182 & -1.177344 & -0.048518 \\
\hline
\end{tabular}

Energy: -415.686407a.u.

Enthalpy: -415.571248a.u. 
Table S38: B3LYP/6-311++G** optimized structure for $\mathbf{F F}^{\text {ts }}-\left(\mathrm{H}_{2} \mathrm{O}\right)_{1}$. Standard orientation:

\begin{tabular}{|c|c|c|c|c|}
\hline \multirow{2}{*}{$\begin{array}{l}\text { Center } \\
\text { Number }\end{array}$} & \multirow{2}{*}{$\begin{array}{l}\text { Atomic } \\
\text { Number }\end{array}$} & \multicolumn{3}{|c|}{ Coordinates (Angstroms) } \\
\hline & & $\mathrm{X}$ & $\mathrm{Y}$ & Z \\
\hline 1 & 6 & 0.262819 & 1.782768 & 0.122115 \\
\hline 2 & 8 & -0.618448 & 0.977493 & 0.518394 \\
\hline 3 & 7 & 1.466667 & 1.500083 & -0.295048 \\
\hline 4 & 1 & 1.822375 & 0.269882 & -0.276510 \\
\hline 5 & 1 & 2.026587 & 2.299153 & -0.563904 \\
\hline 6 & 1 & -0.051379 & 2.840896 & 0.147525 \\
\hline 7 & 7 & 1.944646 & -0.996549 & -0.193063 \\
\hline 8 & 6 & 0.877737 & -1.702082 & 0.071091 \\
\hline 9 & 1 & 2.783139 & -1.536189 & -0.366114 \\
\hline 10 & 8 & -0.282279 & -1.288070 & 0.316406 \\
\hline 11 & 1 & 0.969842 & -2.802779 & 0.090514 \\
\hline 12 & 1 & -2.951747 & -1.138361 & -0.335674 \\
\hline 13 & 8 & -3.210975 & -0.213245 & -0.382658 \\
\hline 14 & 1 & -2.427719 & 0.249125 & -0.055433 \\
\hline
\end{tabular}

Energy: -415.679257a.u.

Enthalpy: -415.568540a.u.

Imaginary Frequency: $-1297.2610 \mathrm{~cm}^{-1}$

Table S39: B3LYP/6-311++G** optimized structure for FF- $\left(\mathrm{H}_{2} \mathrm{O}\right)_{2}$. Standard orientation:

\begin{tabular}{|c|c|c|c|c|}
\hline \multirow{2}{*}{$\begin{array}{l}\text { Center } \\
\text { Number }\end{array}$} & \multirow{2}{*}{$\begin{array}{l}\text { Atomic } \\
\text { Number }\end{array}$} & \multicolumn{3}{|c|}{ Coordinates (Angstroms) } \\
\hline & & $\mathrm{X}$ & Y & Z \\
\hline 1 & 6 & 1.708063 & -1.498977 & -0.381288 \\
\hline 2 & 8 & 0.465657 & -1.298113 & -0.607748 \\
\hline 3 & 7 & 2.630143 & -0.686342 & -0.012155 \\
\hline 4 & 1 & 2.056530 & 0.969988 & 0.483430 \\
\hline 5 & 1 & 3.522383 & -1.161626 & 0.085697 \\
\hline 6 & 1 & 1.918654 & -2.575195 & -0.562239 \\
\hline 7 & 7 & 1.473904 & 1.814287 & 0.651735 \\
\hline 8 & 6 & 0.253947 & 1.867927 & 0.128414 \\
\hline 9 & 1 & 1.811526 & 2.580416 & 1.212233 \\
\hline 10 & 8 & -0.269906 & 0.985906 & -0.560850 \\
\hline 11 & 1 & -0.309037 & 2.790126 & 0.342674 \\
\hline 12 & 1 & -2.656630 & -0.938988 & 0.766946 \\
\hline 13 & 8 & -2.126574 & -1.601499 & 1.237882 \\
\hline
\end{tabular}




$\begin{array}{rrrrr}14 & 1 & -1.302507 & -1.617865 & 0.738561 \\ 15 & 1 & -2.129464 & 0.717819 & -0.739091 \\ 16 & 8 & -3.064418 & 0.521251 & -0.555469 \\ 17 & 1 & -3.449903 & 0.265658 & -1.398545\end{array}$

Energy: -492.1567833a.u.

Enthalpy: -492.013484a.u.

Table S40: B3LYP/6-311++G** optimized structure for $\mathbf{F F}^{\text {ts }}-\left(\mathrm{H}_{2} \mathrm{O}\right)_{2}$. Standard orientation:

\begin{tabular}{|c|c|c|c|c|}
\hline \multirow{2}{*}{$\begin{array}{l}\text { Center } \\
\text { Number }\end{array}$} & \multirow{2}{*}{$\begin{array}{l}\text { Atomic } \\
\text { Number }\end{array}$} & \multicolumn{3}{|c|}{ Coordinates (Angstroms) } \\
\hline & & $\mathrm{X}$ & Y & Z \\
\hline 1 & 6 & -1.148191 & 1.728152 & -0.292025 \\
\hline 2 & 8 & -0.090865 & 1.124091 & -0.610001 \\
\hline 3 & 7 & -2.261834 & 1.216112 & 0.152083 \\
\hline 4 & 1 & -2.306311 & -0.036532 & 0.357342 \\
\hline 5 & 1 & -2.992684 & 1.886459 & 0.354150 \\
\hline 6 & 1 & -1.084793 & 2.822884 & -0.420508 \\
\hline 7 & 7 & -2.121676 & -1.301778 & 0.486255 \\
\hline 8 & 6 & -0.992002 & -1.793612 & 0.061218 \\
\hline 9 & 1 & -2.760978 & -1.976935 & 0.886227 \\
\hline 10 & 8 & -0.040111 & -1.166685 & -0.476184 \\
\hline 11 & 1 & -0.817678 & -2.878657 & 0.164804 \\
\hline 12 & 1 & 2.986129 & 0.620473 & 0.559781 \\
\hline 13 & 8 & 2.739942 & 1.556128 & 0.630949 \\
\hline 14 & 1 & 1.887064 & 1.585825 & 0.182299 \\
\hline 15 & 1 & 1.878920 & -1.289483 & -0.274174 \\
\hline 16 & 8 & 2.822553 & -1.296939 & -0.047379 \\
\hline 17 & 1 & 3.283907 & -1.474383 & -0.872523 \\
\hline
\end{tabular}

Energy: -492.1491687a.u.

Enthalpy: -492.010432a.u.

Imaginary Frequency: $-1287.2631 \mathrm{~cm}^{-1}$

Table S41: B3LYP/6-311++G** optimized structure for FF- $\left(\mathrm{H}_{2} \mathrm{O}\right)_{3}$. Standard orientation:

\begin{tabular}{|c|c|c|c|c|}
\hline \multirow{2}{*}{$\begin{array}{l}\text { Center } \\
\text { Number }\end{array}$} & \multirow{2}{*}{$\begin{array}{l}\text { Atomic } \\
\text { Number }\end{array}$} & \multicolumn{3}{|c|}{ Coordinates (Angstroms) } \\
\hline & & $\mathrm{X}$ & $\mathrm{Y}$ & Z \\
\hline 1 & 6 & -1.460176 & 1.853601 & 0.120738 \\
\hline 2 & 8 & -0.509906 & 1.068544 & 0.203321 \\
\hline
\end{tabular}




\begin{tabular}{rrrrc}
3 & 7 & -2.743783 & 1.519343 & 0.043612 \\
4 & 1 & -3.004845 & 0.511675 & 0.022961 \\
5 & 1 & -3.441879 & 2.242035 & -0.031825 \\
6 & 1 & -1.256177 & 2.936418 & 0.109781 \\
7 & 7 & -2.970745 & -1.295090 & -0.000824 \\
8 & 6 & -1.816502 & -1.844613 & 0.114814 \\
9 & 1 & -3.705252 & -1.991084 & -0.087081 \\
10 & 8 & -0.658362 & -1.321837 & 0.250106 \\
11 & 1 & -1.700420 & -2.949947 & 0.110418 \\
12 & 1 & 1.375768 & 1.481015 & -0.237475 \\
13 & 8 & 2.239885 & 1.511721 & -0.674437 \\
14 & 1 & 2.884688 & 1.289938 & 0.017366 \\
15 & 1 & 1.292402 & -1.462128 & -0.614859 \\
16 & 8 & 2.187246 & -1.319965 & -0.948897 \\
17 & 1 & 2.186630 & -0.377852 & -1.205967 \\
18 & 1 & 3.429224 & -0.836865 & 0.403532 \\
19 & 8 & 3.934829 & -0.178343 & 0.920077 \\
20 & 1 & 4.052097 & -0.547873 & 1.798959 \\
\hline---------------------------
\end{tabular}

Energy: -568.6302378a.u.

Enthalpy: -568.458676a.u.

Table S42: B3LYP/6-311++G** optimized structure for $\mathbf{F F}^{\text {ts }}-\left(\mathrm{H}_{2} \mathrm{O}\right)_{3}$. Standard orientation:

\begin{tabular}{ccccc} 
Center & Atomic & \multicolumn{3}{c}{ Coordinates (Angstroms) } \\
Number & Number & $\mathrm{X}$ & $\mathrm{Y}$ & $\mathrm{Z}$ \\
\hline- & 6 & -1.590332 & 1.779533 & 0.162260 \\
\hline 1 & 8 & -0.527467 & 1.113039 & 0.265525 \\
2 & 7 & -2.806876 & 1.326526 & 0.041566 \\
3 & 1 & -2.973494 & 0.060917 & -0.008186 \\
4 & 1 & -3.525132 & 2.036307 & -0.027404 \\
5 & 1 & -1.434758 & 2.872431 & 0.181438 \\
6 & 7 & -2.904022 & -1.214378 & -0.037437 \\
7 & 6 & -1.728945 & -1.765318 & 0.088180 \\
8 & 1 & -3.672760 & -1.862259 & -0.154518 \\
9 & 8 & -0.621969 & -1.188110 & 0.247442 \\
10 & 1 & -1.658265 & -2.866771 & 0.057974 \\
11 & 1 & 1.368446 & 1.454947 & -0.427803 \\
12 & 8 & 2.215219 & 1.414662 & -0.892611 \\
13 & 1 & 2.880445 & 1.291000 & -0.194789 \\
14 & 1 & 1.314432 & -1.530139 & -0.361085 \\
15 & 8 & 2.203787 & -1.442373 & -0.730753 \\
16 & & & &
\end{tabular}




$\begin{array}{llrrc}17 & 1 & 2.185803 & -0.560370 & -1.146847 \\ 18 & 1 & 3.455067 & -0.735784 & 0.494942 \\ 19 & 8 & 3.962484 & 0.001427 & 0.890297 \\ 20 & 1 & 4.095727 & -0.219769 & 1.815529\end{array}$

Energy: -568.6233077a.u.

Enthalpy: -568.456243a.u.

Imaginary Frequency: $-1291.5964 \mathrm{~cm}^{-1}$

Table S43: B3LYP/6-311++G** optimized structure for FF- $\left(\mathrm{H}_{2} \mathrm{O}\right)_{4}$. Standard orientation:

\begin{tabular}{|c|c|c|c|c|}
\hline \multirow{2}{*}{$\begin{array}{l}\text { Center } \\
\text { Number }\end{array}$} & \multirow{2}{*}{$\begin{array}{l}\text { Atomic } \\
\text { Number }\end{array}$} & \multicolumn{3}{|c|}{ Coordinates (Angstroms) } \\
\hline & & $\mathrm{X}$ & Y & $\mathrm{Z}$ \\
\hline 1 & 6 & 2.138318 & -1.827917 & -0.053855 \\
\hline 2 & 8 & 0.993116 & -1.304160 & -0.271694 \\
\hline 3 & 7 & 3.276855 & -1.276966 & 0.165429 \\
\hline 4 & 1 & 3.309855 & 0.530757 & 0.122186 \\
\hline 5 & 1 & 4.005450 & -1.972393 & 0.295333 \\
\hline 6 & 1 & 2.025023 & -2.933082 & -0.080190 \\
\hline 7 & 7 & 3.048038 & 1.536768 & 0.064554 \\
\hline 8 & 6 & 1.769604 & 1.863705 & -0.092093 \\
\hline 9 & 1 & 3.740387 & 2.263661 & 0.151354 \\
\hline 10 & 8 & 0.828011 & 1.071610 & -0.201308 \\
\hline 11 & 1 & 1.561704 & 2.945297 & -0.125277 \\
\hline 12 & 1 & -1.024315 & -1.649209 & 0.201785 \\
\hline 13 & 8 & -1.963340 & -1.798362 & 0.372132 \\
\hline 14 & 1 & -2.408640 & -1.495742 & -0.445545 \\
\hline 15 & 1 & -3.004566 & -0.302622 & -2.670924 \\
\hline 16 & 8 & -3.102996 & -0.353979 & -1.716868 \\
\hline 17 & 1 & -2.772122 & 0.497699 & -1.349126 \\
\hline 18 & 1 & -2.705564 & 0.201900 & 2.894866 \\
\hline 19 & 8 & -2.803391 & 0.306966 & 1.945563 \\
\hline 20 & 1 & -2.536098 & -0.543009 & 1.529562 \\
\hline 21 & 1 & -1.066092 & 1.551811 & -0.297651 \\
\hline 22 & 8 & -2.015122 & 1.751516 & -0.318316 \\
\hline 23 & 1 & -2.337036 & 1.482868 & 0.563358 \\
\hline
\end{tabular}

Energy: -645.1084737a.u.

Enthalpy: -644.908722a.u.

Table S44: B3LYP/6-311++G** optimized structure for $\mathbf{F F}^{\text {ts }}-\left(\mathrm{H}_{2} \mathrm{O}\right)_{4}$. Standard orientation: 


\begin{tabular}{|c|c|c|c|c|}
\hline \multirow{2}{*}{$\begin{array}{l}\text { Center } \\
\text { Number }\end{array}$} & \multirow{2}{*}{$\begin{array}{l}\text { Atomic } \\
\text { Number }\end{array}$} & \multicolumn{3}{|c|}{ Coordinates (Angstroms) } \\
\hline & & $\mathrm{X}$ & $\mathrm{Y}$ & Z \\
\hline 1 & 6 & 1.987213 & 1.771805 & 0.003904 \\
\hline 2 & 8 & 0.896496 & 1.144629 & -0.022031 \\
\hline 3 & 7 & 3.191615 & 1.272199 & 0.023411 \\
\hline 4 & 1 & 3.311560 & 0.000111 & 0.000456 \\
\hline 5 & 1 & 3.939879 & 1.953459 & 0.043136 \\
\hline 6 & 1 & 1.870405 & 2.869599 & 0.010157 \\
\hline 7 & 7 & 3.191839 & -1.271985 & -0.022649 \\
\hline 8 & 6 & 1.987493 & -1.771771 & -0.004513 \\
\hline 9 & 1 & 3.940231 & -1.953130 & -0.041497 \\
\hline 10 & 8 & 0.896657 & -1.144752 & 0.020126 \\
\hline 11 & 1 & 1.870852 & -2.869582 & -0.010857 \\
\hline 12 & 1 & -1.063926 & 1.623175 & -0.168531 \\
\hline 13 & 8 & -2.012915 & 1.795557 & -0.244631 \\
\hline 14 & 1 & -2.389664 & 1.458351 & 0.592224 \\
\hline 15 & 1 & -2.861275 & 0.095541 & 2.796695 \\
\hline 16 & 8 & -2.975373 & 0.225230 & 1.852048 \\
\hline 17 & 1 & -2.676813 & -0.601774 & 1.409780 \\
\hline 18 & 1 & -2.863359 & -0.095712 & -2.795702 \\
\hline 19 & 8 & -2.976980 & -0.225295 & -1.850981 \\
\hline 20 & 1 & -2.678048 & 0.601710 & -1.408968 \\
\hline 21 & 1 & -1.063758 & -1.623225 & 0.167934 \\
\hline 22 & 8 & -2.012678 & -1.795603 & 0.244863 \\
\hline 23 & 1 & -2.390155 & -1.458365 & -0.591657 \\
\hline
\end{tabular}

Energy: -645.1015247a.u.

Enthalpy: -644.906338a.u.

Imaginary Frequency: $-1290.7967 \mathrm{~cm}^{-1}$

Table S45: B3LYP/6-311++G** optimized structure for FF-N- $\left(\mathrm{H}_{2} \mathrm{O}\right)_{2}$. Standard orientation:

\begin{tabular}{|c|c|c|c|c|}
\hline \multirow{2}{*}{$\begin{array}{l}\text { Center } \\
\text { Number }\end{array}$} & \multirow{2}{*}{$\begin{array}{l}\text { Atomic } \\
\text { Number }\end{array}$} & \multicolumn{3}{|c|}{ Coordinates (Angstroms) } \\
\hline & & $\mathrm{X}$ & Y & Z \\
\hline 1 & 6 & 1.781896 & -0.770166 & -0.035071 \\
\hline 2 & 8 & 1.274023 & -1.941051 & -0.046920 \\
\hline 3 & 7 & 1.233780 & 0.392775 & -0.002277 \\
\hline 4 & 1 & -0.585261 & 0.366838 & 0.003214 \\
\hline 5 & 1 & 1.956234 & 1.109476 & -0.003340 \\
\hline 6 & 1 & 2.890133 & -0.862031 & -0.058598 \\
\hline
\end{tabular}




\begin{tabular}{rrrrc}
7 & 7 & -1.586853 & 0.086523 & 0.008472 \\
8 & 6 & -1.888640 & -1.209080 & 0.030964 \\
9 & 1 & -2.332054 & 0.773297 & 0.005021 \\
10 & 8 & -1.100189 & -2.157062 & 0.039478 \\
11 & 1 & -2.972325 & -1.423558 & 0.042367 \\
12 & 1 & -4.020765 & 2.717840 & 0.728242 \\
13 & 8 & -3.813127 & 2.145490 & -0.017377 \\
14 & 1 & -4.067361 & 2.637072 & -0.804998 \\
15 & 1 & 4.485334 & 2.138597 & 0.812040 \\
16 & 8 & 4.044927 & 1.809717 & 0.021951 \\
17 & 1 & 4.512969 & 2.206100 & -0.719729 \\
\hline & 1 & & &
\end{tabular}

Energy: -492.1500565a.u.

Enthalpy: -492.007317a.u.

Table S46: B3LYP/6-311++G** optimized structure for $\mathbf{F F}^{\mathrm{ts}}-\mathrm{N}-\left(\mathrm{H}_{2} \mathrm{O}\right)_{2}$. Standard orientation:

\begin{tabular}{|c|c|c|c|c|}
\hline \multirow{2}{*}{$\begin{array}{l}\text { Center } \\
\text { Number }\end{array}$} & \multirow{2}{*}{$\begin{array}{l}\text { Atomic } \\
\text { Number }\end{array}$} & \multicolumn{3}{|c|}{ Coordinates (Angstroms) } \\
\hline & & $X$ & Y & Z \\
\hline 1 & 6 & -1.765395 & -1.045364 & 0.000118 \\
\hline 2 & 8 & -1.152493 & -2.142618 & 0.000165 \\
\hline 3 & 7 & -1.273941 & 0.164042 & 0.000009 \\
\hline 4 & 1 & 0.000005 & 0.281199 & -0.000003 \\
\hline 5 & 1 & -1.972008 & 0.902017 & -0.000009 \\
\hline 6 & 1 & -2.866551 & -1.150760 & 0.000178 \\
\hline 7 & 7 & 1.273949 & 0.164036 & -0.000014 \\
\hline 8 & 6 & 1.765398 & -1.045373 & -0.000118 \\
\hline 9 & 1 & 1.972020 & 0.902008 & 0.000004 \\
\hline 10 & 8 & 1.152490 & -2.142624 & -0.000163 \\
\hline 11 & 1 & 2.866553 & -1.150775 & -0.000177 \\
\hline 12 & 1 & -4.000900 & 2.611538 & -0.767709 \\
\hline 13 & 8 & -3.657131 & 2.143723 & -0.000079 \\
\hline 14 & 1 & -4.000893 & 2.611695 & 0.767459 \\
\hline 15 & 1 & 4.000880 & 2.611551 & 0.767715 \\
\hline 16 & 8 & 3.657127 & 2.143730 & 0.000081 \\
\hline 17 & 1 & 4.000883 & 2.611714 & -0.767453 \\
\hline
\end{tabular}

Energy: -492.1425798a.u.

Enthalpy: -492.004488a.u.

Imaginary Frequency: $-1319.4464 \mathrm{~cm}^{-1}$

Table S47: B3LYP/6-311++G** optimized structure for FF- $\left(\mathrm{H}_{2} \mathrm{O}\right)_{5}$. Standard orientation: 


\begin{tabular}{|c|c|c|c|c|}
\hline \multirow{2}{*}{$\begin{array}{l}\text { Center } \\
\text { Number }\end{array}$} & \multirow{2}{*}{$\begin{array}{l}\text { Atomic } \\
\text { Number }\end{array}$} & \multicolumn{3}{|c|}{ Coordinates (Angstroms) } \\
\hline & & $\mathrm{X}$ & $\mathrm{Y}$ & Z \\
\hline 1 & 6 & -0.368513 & 0.194877 & -2.566395 \\
\hline 2 & 8 & -0.349179 & 0.173446 & -1.284656 \\
\hline 3 & 7 & 0.586183 & 0.376241 & -3.403245 \\
\hline 4 & 1 & 2.228797 & 0.651688 & -2.691458 \\
\hline 5 & 1 & 0.250363 & 0.338441 & -4.361091 \\
\hline 6 & 1 & -1.413355 & 0.020292 & -2.900147 \\
\hline 7 & 7 & 3.031008 & 0.780089 & -2.040285 \\
\hline 8 & 6 & 2.823032 & 0.698160 & -0.730956 \\
\hline 9 & 1 & 3.959020 & 0.966915 & -2.385818 \\
\hline 10 & 8 & 1.738652 & 0.475398 & -0.181746 \\
\hline 11 & 1 & 3.716239 & 0.846482 & -0.103201 \\
\hline 12 & 1 & -1.214392 & 1.730136 & 0.409008 \\
\hline 13 & 8 & -1.614072 & 1.682202 & 1.284966 \\
\hline 14 & 1 & -0.877640 & 1.362593 & 1.857551 \\
\hline 15 & 1 & 1.135243 & 0.511157 & 1.733992 \\
\hline 16 & 8 & 0.607809 & 0.623566 & 2.537988 \\
\hline 17 & 1 & 0.414236 & -0.285920 & 2.841247 \\
\hline 18 & 1 & -4.156815 & -0.301557 & 1.590960 \\
\hline 19 & 8 & -3.472396 & -0.276400 & 0.916537 \\
\hline 20 & 1 & -2.890253 & 0.489772 & 1.136750 \\
\hline 21 & 1 & -0.894473 & -1.573800 & -0.021880 \\
\hline 22 & 8 & -1.432568 & -2.119064 & 0.565885 \\
\hline 23 & 1 & -2.250619 & -1.591355 & 0.693237 \\
\hline 24 & 1 & -0.642556 & -2.177176 & 2.141749 \\
\hline 25 & 8 & -0.186241 & -2.010635 & 2.998737 \\
\hline 26 & 1 & 0.252715 & -2.828305 & 3.246221 \\
\hline
\end{tabular}

Energy: -721.5838965a.u.

Enthalpy: -721.356029 a.u.

Table S48: B3LYP/6-311++G** optimized structure for $\mathbf{F F}^{\text {ts }}-\left(\mathrm{H}_{2} \mathrm{O}\right)_{5}$. Standard orientation:

\begin{tabular}{|c|c|c|c|c|}
\hline \multirow{2}{*}{$\begin{array}{l}\text { Center } \\
\text { Number }\end{array}$} & \multirow{2}{*}{$\begin{array}{l}\text { Atomic } \\
\text { Number }\end{array}$} & \multicolumn{3}{|c|}{ Coordinates (Angstroms) } \\
\hline & & $\mathrm{X}$ & Y & Z \\
\hline 1 & 6 & -1.647472 & -1.832247 & -0.348598 \\
\hline 2 & 8 & -0.767738 & -0.945984 & -0.159006 \\
\hline 3 & 7 & -2.938644 & -1.668093 & -0.375997 \\
\hline 4 & 1 & -3.393025 & -0.492546 & -0.173791 \\
\hline
\end{tabular}




\begin{tabular}{rrrrr}
5 & 1 & -3.477231 & -2.509856 & -0.537919 \\
6 & 1 & -1.235838 & -2.844055 & -0.503989 \\
7 & 7 & -3.619249 & 0.748349 & 0.043056 \\
8 & 6 & -2.598737 & 1.550929 & 0.155915 \\
9 & 1 & -4.525572 & 1.192313 & 0.121266 \\
10 & 8 & -1.375901 & 1.256278 & 0.094725 \\
11 & 1 & -2.787764 & 2.625260 & 0.326071 \\
12 & 1 & 0.517760 & -0.605154 & 1.635547 \\
13 & 8 & 1.368272 & -0.382642 & 2.034639 \\
14 & 1 & 1.496996 & 0.557953 & 1.777329 \\
15 & 1 & 0.486535 & 2.121073 & 0.599647 \\
16 & 8 & 1.379860 & 2.182365 & 0.961491 \\
17 & 1 & 1.966226 & 2.184152 & 0.177629 \\
18 & 1 & 3.867728 & -2.098047 & 0.842427 \\
19 & 8 & 2.946782 & -2.057222 & 0.570318 \\
20 & 1 & 2.490125 & -1.464084 & 1.212995 \\
21 & 1 & 1.007236 & -0.729043 & -1.341391 \\
22 & 8 & 1.917497 & -0.765709 & -1.664795 \\
23 & 1 & 2.386747 & -1.298133 & -0.987413 \\
24 & 1 & 2.596480 & 0.854583 & -1.564321 \\
25 & 8 & 2.901087 & 1.762222 & -1.330126 \\
26 & 1 & 3.027239 & 2.237225 & -2.155366 \\
\hline-------------------------------------------------------------------
\end{tabular}

Energy: -721.5768439a.u.

Enthalpy: -721.353443a.u.

Imaginary Frequency: $-1292.2931 \mathrm{~cm}^{-1}$

Table S49: B3LYP/6-311++G** optimized structure for FF- $\left(\mathrm{H}_{2} \mathrm{O}\right)_{6}$. Standard orientation:

\begin{tabular}{ccccc} 
Center & Atomic & \multicolumn{3}{c}{ Coordinates (Angstroms) } \\
Number & Number & X & Y & $Z$ \\
---1 & -0.684725 & 0.002448 & -2.894942 \\
1 & 6 & -0.662057 & 0.001928 & -1.614401 \\
2 & 8 & 0.273340 & 0.003081 & -3.744873 \\
3 & 7 & 1.946858 & 0.003661 & -3.079388 \\
4 & 1 & -0.070629 & 0.003391 & -4.700586 \\
5 & 1 & -1.746251 & 0.002264 & -3.225924 \\
6 & 1 & 2.786600 & 0.003717 & -2.463082 \\
7 & 7 & 2.626816 & 0.002307 & -1.148303 \\
8 & 6 & 3.717410 & 0.004720 & -2.850393 \\
9 & 1 & 1.542207 & 0.000964 & -0.546643 \\
10 & 8 & 3.558002 & 0.002378 & -0.560440 \\
11 & 1 & & &
\end{tabular}




$\begin{array}{llrrr}12 & 1 & -1.532138 & 1.495612 & -0.270499 \\ 13 & 8 & -1.769917 & 2.034202 & 0.493770 \\ 14 & 1 & -0.074796 & 2.231584 & 1.329059 \\ 15 & 1 & 1.169998 & 1.413269 & 1.014674 \\ 16 & 8 & 0.795324 & 1.982948 & 1.699340 \\ 17 & 1 & 0.241615 & 0.766019 & 3.051626 \\ 18 & 1 & -1.530601 & -1.493756 & -0.273761 \\ 19 & 8 & -1.768903 & -2.034906 & 0.488546 \\ 20 & 1 & -2.210466 & -1.417657 & 1.102980 \\ 21 & 1 & 1.171080 & -1.416019 & 1.011822 \\ 22 & 8 & 0.795914 & -1.987111 & 1.695026 \\ 23 & 1 & -0.074141 & -2.234604 & 1.323815 \\ 24 & 1 & 0.241785 & -0.773255 & 3.050006 \\ 25 & 8 & -0.193884 & -0.004109 & 3.469582 \\ 26 & 1 & -1.727281 & -0.003476 & 2.851240 \\ 27 & 1 & -3.307103 & -0.003664 & 2.919843 \\ 28 & 8 & -2.567985 & -0.002857 & 2.304104 \\ 29 & 1 & -2.211057 & 1.414938 & 1.106488\end{array}$

Energy: -798.0575319a.u.

Enthalpy: -797.801284a.u.

Table S50: B3LYP/6-311++G** optimized structure for $\mathbf{F F}^{\text {ts }}-\left(\mathrm{H}_{2} \mathrm{O}\right)_{6}$. Standard orientation:

\begin{tabular}{ccccc} 
Center & Atomic & \multicolumn{3}{c}{ Coordinates (Angstroms) } \\
Number & Number & $\mathrm{X}$ & $\mathrm{Y}$ & $\mathrm{Z}$ \\
- & - & 2.240664 & -1.802329 & -0.000743 \\
1 & 6 & 1.149140 & -1.164462 & -0.001291 \\
2 & 8 & 3.443975 & -1.310031 & 0.000023 \\
3 & 7 & 3.576204 & -0.036937 & 0.000128 \\
4 & 1 & 4.190625 & -1.993970 & 0.000369 \\
5 & 1 & 2.118660 & -2.899155 & -0.000952 \\
6 & 1 & 3.483734 & 1.233473 & 0.000185 \\
7 & 7 & 2.297783 & 1.767039 & 0.000225 \\
8 & 6 & 4.253558 & 1.891130 & 0.000395 \\
9 & 1 & 1.182761 & 1.173989 & 0.000033 \\
10 & 8 & 2.213929 & 2.867798 & 0.000448 \\
11 & 1 & -0.406680 & -1.434146 & -1.410994 \\
12 & 1 & -1.178877 & -1.360338 & -1.986566 \\
13 & 8 & -1.246019 & 0.504012 & -2.227036 \\
14 & 1 & -0.423781 & 1.558844 & -1.499363 \\
15 & 1 & -1.228862 & 1.461183 & -2.022284 \\
16 & 8 & & &
\end{tabular}




$\begin{array}{rrrrr}17 & 1 & -2.658805 & 1.539208 & -0.769187 \\ 18 & 1 & -0.406412 & -1.436075 & 1.407348 \\ 19 & 8 & -1.177623 & -1.363438 & 1.984401 \\ 20 & 1 & -1.941871 & -1.513228 & 1.396027 \\ 21 & 1 & -0.422629 & 1.556482 & 1.501285 \\ 22 & 8 & -1.227429 & 1.458124 & 2.024510 \\ 23 & 1 & -1.244521 & 0.500674 & 2.227896 \\ 24 & 1 & -2.658347 & 1.538079 & 0.772449 \\ 25 & 8 & -3.221806 & 1.325191 & 0.001658 \\ 26 & 1 & -3.338088 & -0.327998 & 0.000398 \\ 27 & 1 & -4.085182 & -1.721578 & -0.000832 \\ 28 & 8 & -3.210185 & -1.322054 & -0.000394 \\ 29 & 1 & -1.942236 & -1.511039 & -1.397257\end{array}$

Energy: -798.050975a.u.

Enthalpy: -797.799142a.u.

Imaginary Frequency: $-1288.9 \mathrm{~cm}^{-1}$

Table S51: B3LYP/6-311++G** optimized structure for FF- $\left(\mathrm{H}_{2} \mathrm{O}\right)_{4}-\mathrm{N}-\left(\mathrm{H}_{2} \mathrm{O}\right)_{2}$. Standard orientation:

\begin{tabular}{ccccc} 
Center & Atomic & \multicolumn{3}{c}{ Coordinates (Angstroms) } \\
Number & Number & X & Y & $Z$ \\
\hline 1 & 6 & -1.143536 & 1.853477 & -0.232664 \\
2 & 8 & -0.028464 & 1.269643 & -0.456689 \\
3 & 7 & -2.311892 & 1.384183 & 0.009621 \\
4 & 1 & -2.443488 & -0.446514 & -0.014462 \\
5 & 1 & -2.968623 & 2.152270 & 0.130157 \\
6 & 1 & -0.988814 & 2.954298 & -0.274281 \\
7 & 7 & -2.248812 & -1.465364 & -0.058663 \\
8 & 6 & -0.993640 & -1.867802 & -0.195823 \\
9 & 1 & -2.986464 & -2.157226 & 0.021200 \\
10 & 8 & 0.003912 & -1.140938 & -0.299480 \\
11 & 1 & -0.858190 & -2.961476 & -0.216349 \\
12 & 1 & 1.923247 & 1.496079 & 0.141755 \\
13 & 8 & 2.855337 & 1.601538 & 0.377479 \\
14 & 1 & 3.340091 & 1.254877 & -0.399241 \\
15 & 1 & 3.916363 & 0.012785 & -2.566108 \\
16 & 8 & 4.056262 & 0.056337 & -1.616661 \\
17 & 1 & 3.653450 & -0.759874 & -1.236868 \\
18 & 1 & 3.083438 & -0.391340 & 2.957904 \\
19 & 8 & 3.466610 & -0.482863 & 2.081875 \\
20 & 1 & 3.270038 & 0.354081 & 1.602278 \\
& & & &
\end{tabular}




$\begin{array}{llrrr}21 & 1 & 1.837467 & -1.707300 & -0.288674 \\ 22 & 8 & 2.779289 & -1.941217 & -0.231258 \\ 23 & 1 & 3.043643 & -1.653714 & 0.663774 \\ 24 & 8 & -4.229852 & -3.698156 & 0.154255 \\ 25 & 1 & -4.757273 & -4.069725 & -0.560059 \\ 26 & 1 & -4.562378 & -4.096437 & 0.964997 \\ 27 & 1 & -3.427047 & 4.998789 & -0.503044 \\ 28 & 8 & -3.058613 & 4.426897 & 0.178190 \\ 29 & 1 & -3.083332 & 4.944701 & 0.989560\end{array}$

Energy: -798.0432477a.u.

Enthalpy: -797.788318a.u.

Table S52: B3LYP/6-311++G** optimized structure for $\mathbf{F F}^{\text {ts }}-\left(\mathrm{H}_{2} \mathrm{O}\right)_{4}-\mathrm{N}-\left(\mathrm{H}_{2} \mathrm{O}\right)_{2}$. Standard orientation:

\begin{tabular}{|c|c|c|c|c|}
\hline \multirow{2}{*}{$\begin{array}{l}\text { Center } \\
\text { Number }\end{array}$} & \multirow{2}{*}{$\begin{array}{l}\text { Atomic } \\
\text { Number }\end{array}$} & \multicolumn{3}{|c|}{ Coordinates (Angstroms) } \\
\hline & & $X$ & Y & Z \\
\hline 1 & 6 & -1.050458 & -0.030771 & -1.745179 \\
\hline 2 & 8 & -1.047104 & -0.031845 & -0.483004 \\
\hline 3 & 7 & -0.031307 & -0.028153 & -2.552692 \\
\hline 4 & 1 & 1.136447 & -0.041668 & -2.030633 \\
\hline 5 & 1 & -0.275596 & -0.027037 & -3.539766 \\
\hline 6 & 1 & -2.062957 & -0.032287 & -2.186897 \\
\hline 7 & 7 & 2.191890 & -0.050871 & -1.307897 \\
\hline 8 & 6 & 2.036996 & -0.003258 & -0.017744 \\
\hline 9 & 1 & 3.160718 & -0.079733 & -1.615361 \\
\hline 10 & 8 & 0.959793 & 0.038985 & 0.638722 \\
\hline 11 & 1 & 2.943038 & 0.002332 & 0.614180 \\
\hline 12 & 1 & -2.398877 & -0.124023 & 0.963968 \\
\hline 13 & 8 & -3.010278 & -0.175475 & 1.714009 \\
\hline 14 & 1 & -2.876232 & 0.664855 & 2.195502 \\
\hline 15 & 1 & -1.844175 & 2.861940 & 3.113852 \\
\hline 16 & 8 & -2.070156 & 1.945125 & 3.290099 \\
\hline 17 & 1 & -1.219065 & 1.469256 & 3.432042 \\
\hline 18 & 1 & -1.735001 & -2.731917 & 3.282086 \\
\hline 19 & 8 & -1.752409 & -1.806920 & 3.540463 \\
\hline 20 & 1 & -2.310854 & -1.341009 & 2.875613 \\
\hline 21 & 1 & 0.435647 & 0.197857 & 2.543851 \\
\hline 22 & 8 & 0.117321 & 0.281636 & 3.455263 \\
\hline 23 & 1 & -0.377020 & -0.546166 & 3.617545 \\
\hline 24 & 8 & 5.210535 & -0.137202 & -1.777146 \\
\hline 25 & 1 & 5.792967 & 0.607443 & -1.958207 \\
\hline
\end{tabular}




$\begin{array}{rrrrr}26 & 1 & 5.748944 & -0.926451 & -1.894329 \\ 27 & 1 & -1.376264 & 0.762478 & -5.918632 \\ 28 & 8 & -1.209121 & -0.012464 & -5.372685 \\ 29 & 1 & -1.373687 & -0.773247 & -5.938931\end{array}$

Energy: -798.0355804a.u.

Enthalpy: -797.785312a.u.

Imaginary Frequency: $-1308.1 \mathrm{~cm}^{-1}$

Table S53: B3LYP/6-311++ $\mathrm{G}^{* *}$ optimized structure for FF- $\left(\mathrm{H}_{2} \mathrm{O}\right)_{4}-\mathrm{N}-\left(\mathrm{H}_{2} \mathrm{O}\right)_{4}$. Standard orientation:

\begin{tabular}{|c|c|c|c|c|}
\hline \multirow{2}{*}{$\begin{array}{l}\text { Center } \\
\text { Number }\end{array}$} & \multirow{2}{*}{$\begin{array}{l}\text { Atomic } \\
\text { Number }\end{array}$} & \multicolumn{3}{|c|}{ Coordinates (Angstroms) } \\
\hline & & $\mathrm{X}$ & $\mathrm{Y}$ & $\mathrm{Z}$ \\
\hline 1 & 6 & -0.038396 & 0.016926 & 0.037474 \\
\hline 2 & 8 & -0.024560 & -0.105462 & 1.269980 \\
\hline 3 & 7 & 1.026507 & 0.145054 & -0.740513 \\
\hline 4 & 1 & 1.966123 & 0.094179 & -0.300164 \\
\hline 5 & 1 & 0.952115 & 0.168884 & -1.750222 \\
\hline 6 & 1 & -1.009990 & 0.028093 & -0.481911 \\
\hline 7 & 7 & 3.384203 & -0.137522 & 0.802041 \\
\hline 8 & 6 & 3.092235 & -0.072074 & 2.050420 \\
\hline 9 & 1 & 4.383857 & -0.069996 & 0.621506 \\
\hline 10 & 8 & 1.957792 & -0.083286 & 2.636414 \\
\hline 11 & 1 & 3.886192 & 0.009098 & 2.824700 \\
\hline 12 & 1 & -1.556139 & -0.137383 & 2.474626 \\
\hline 13 & 8 & -2.288449 & -0.133829 & 3.111296 \\
\hline 14 & 1 & -2.253663 & 0.753103 & 3.517808 \\
\hline 15 & 1 & -1.444467 & 3.083589 & 4.561237 \\
\hline 16 & 8 & -1.609422 & 2.138704 & 4.603848 \\
\hline 17 & 1 & -0.782241 & 1.714431 & 4.925158 \\
\hline 18 & 1 & -1.302065 & -2.476278 & 5.168745 \\
\hline 19 & 8 & -1.314865 & -1.521802 & 5.275132 \\
\hline 20 & 1 & -1.774292 & -1.152689 & 4.485407 \\
\hline 21 & 1 & 1.011301 & 0.374503 & 4.443766 \\
\hline 22 & 8 & 0.544491 & 0.549749 & 5.271160 \\
\hline 23 & 1 & 0.023712 & -0.263320 & 5.433021 \\
\hline 24 & 8 & 5.715550 & -0.050060 & -1.293012 \\
\hline 25 & 1 & 5.344544 & 0.656779 & -1.867973 \\
\hline 26 & 1 & 6.654959 & -0.101710 & -1.493297 \\
\hline 27 & 1 & 2.135815 & -0.580655 & -4.291278 \\
\hline 28 & 8 & 2.325530 & -0.235932 & -3.413008 \\
\hline 29 & 1 & 2.789055 & -0.959305 & -2.918243 \\
\hline
\end{tabular}




$\begin{array}{llrrr}30 & 8 & 4.363005 & 1.632418 & -2.983095 \\ 31 & 1 & 3.586306 & 1.094701 & -3.242767 \\ 32 & 1 & 4.027792 & 2.503506 & -2.751132 \\ 33 & 1 & 4.536073 & -1.419728 & -1.653730 \\ 34 & 8 & 3.715804 & -1.925786 & -1.826509 \\ 35 & 1 & 3.292036 & -1.979615 & -0.960515\end{array}$

Energy: -951.0013889a.u.

Enthalpy: -950.689575a.u.

Table S54: B3LYP/6-311++G** optimized structure for $\mathbf{F F}^{\text {ts }}-\left(\mathrm{H}_{2} \mathrm{O}\right)_{4}-\mathrm{N}-\left(\mathrm{H}_{2} \mathrm{O}\right)_{4}$. Standard orientation:

\begin{tabular}{|c|c|c|c|c|}
\hline \multirow{2}{*}{$\begin{array}{l}\text { Center } \\
\text { Number }\end{array}$} & \multirow{2}{*}{$\begin{array}{l}\text { Atomic } \\
\text { Number }\end{array}$} & \multicolumn{3}{|c|}{ Coordinates (Angstroms) } \\
\hline & & $\mathrm{X}$ & $\mathrm{Y}$ & $\mathrm{Z}$ \\
\hline 1 & 6 & -0.019875 & 0.026846 & 0.022676 \\
\hline 2 & 8 & -0.016124 & 0.021236 & 1.283404 \\
\hline 3 & 7 & 1.006854 & 0.032039 & -0.776152 \\
\hline 4 & 1 & 2.167198 & -0.003910 & -0.244270 \\
\hline 5 & 1 & 0.818216 & 0.022066 & -1.774233 \\
\hline 6 & 1 & -1.032852 & 0.027938 & -0.418207 \\
\hline 7 & 7 & 3.226347 & -0.036133 & 0.468301 \\
\hline 8 & 6 & 3.082113 & 0.012135 & 1.760273 \\
\hline 9 & 1 & 4.175856 & -0.054222 & 0.107815 \\
\hline 10 & 8 & 2.005625 & 0.057256 & 2.414959 \\
\hline 11 & 1 & 3.987296 & 0.014964 & 2.393629 \\
\hline 12 & 1 & -1.373334 & -0.095769 & 2.749374 \\
\hline 13 & 8 & -1.984821 & -0.153404 & 3.497925 \\
\hline 14 & 1 & -1.863403 & 0.689914 & 3.977307 \\
\hline 15 & 1 & -0.872791 & 2.901098 & 4.987676 \\
\hline 16 & 8 & -1.064171 & 1.964080 & 5.075230 \\
\hline 17 & 1 & -0.202744 & 1.509211 & 5.219544 \\
\hline 18 & 1 & -0.754826 & -2.689619 & 5.169654 \\
\hline 19 & 8 & -0.712653 & -1.747023 & 5.348617 \\
\hline 20 & 1 & -1.277822 & -1.303178 & 4.675286 \\
\hline 21 & 1 & 1.467569 & 0.243977 & 4.333381 \\
\hline 22 & 8 & 1.150194 & 0.335725 & 5.243559 \\
\hline 23 & 1 & 0.663363 & -0.494179 & 5.416730 \\
\hline 24 & 8 & 5.292660 & 0.003109 & -1.782041 \\
\hline 25 & 1 & 4.816329 & 0.766047 & -2.188820 \\
\hline 26 & 1 & 6.222392 & 0.107256 & -2.006981 \\
\hline 27 & 1 & 1.548279 & -0.240651 & -4.617499 \\
\hline 28 & 8 & 1.844319 & -0.113533 & -3.710832 \\
\hline
\end{tabular}




$\begin{array}{llrrr}29 & 1 & 2.426775 & -0.880339 & -3.493610 \\ 30 & 8 & 3.673059 & 1.846549 & -2.951435 \\ 31 & 1 & 2.946314 & 1.287095 & -3.297409 \\ 32 & 1 & 3.255202 & 2.535900 & -2.425944 \\ 33 & 1 & 4.340112 & -1.402922 & -2.462322 \\ 34 & 8 & 3.655731 & -1.963925 & -2.883920 \\ 35 & 1 & 3.414377 & -2.629475 & -2.232199\end{array}$

Energy: -950.9944462a.u.

Enthalpy: -950.687462a.u.

Imaginary Frequency: $-1295.5143 \mathrm{~cm}^{-1}$

Table S55: B3LYP/6-311++G** optimized structure for Fr1. Standard orientation:

\begin{tabular}{ccccc}
$\begin{array}{l}\text { Center } \\
\text { Number }\end{array}$ & $\begin{array}{l}\text { Atomic } \\
\text { Number }\end{array}$ & \multicolumn{3}{c}{ Coordinates (Angstroms) } \\
\hline-1 & 1 & 0.148976 & 1.522715 & 0.044722 \\
2 & 6 & 0.083293 & 0.426912 & 0.043920 \\
3 & 8 & 1.084018 & -0.287815 & -0.021895 \\
4 & 7 & -1.168031 & -0.100076 & -0.054590 \\
5 & 1 & -1.144661 & -1.081136 & 0.249046
\end{tabular}

Energy: $\quad-169.263416350 \mathrm{au}$

Table S56: B3LYP/6-311++G** optimized structure for Fr2. Standard orientation:

\begin{tabular}{ccccc}
$\begin{array}{l}\text { Center } \\
\text { Number }\end{array}$ & Atomic & Number & \multicolumn{3}{c}{ Coordinates (Angstroms) } \\
- & $\mathrm{X}$ & $\mathrm{Y}$ & $\mathrm{Z}$ \\
\hline 1 & 1 & -0.042590 & 1.467895 & -0.041583 \\
2 & 6 & -0.100721 & 0.354227 & -0.010069 \\
3 & 8 & -1.146046 & -0.242965 & 0.028746 \\
4 & 7 & 1.157663 & -0.224973 & -0.122420 \\
5 & 1 & 1.711644 & -0.074729 & 0.728973
\end{tabular}

Energy: -169.259431669 au

Table S57: B3LYP/6-311++G** optimized structure for $\mathbf{F}$. Standard orientation:

Center Atomic Coordinates (Angstroms)




$\begin{array}{ccccc}\text { Number } & \text { Number } & \text { X } & Y & Z \\ ----------------------------------------------------------------- \\ 1 & 1 & 0.140540 & 1.492474 & -0.000034 \\ 2 & 6 & 0.162882 & 0.386715 & -0.000039 \\ 3 & 8 & 1.196174 & -0.246397 & 0.000109 \\ 4 & 7 & -1.084439 & -0.157330 & -0.000477 \\ 5 & 1 & -1.187757 & -1.161280 & 0.000835 \\ 6 & 1 & -1.908394 & 0.421003 & 0.001902\end{array}$

Energy: $\quad-169.954851288 \mathrm{au}$ 